\title{
Modeling and Analyzing HIV Transmission: The Effect of Contact Patterns
}

\author{
JOHN A. JACQUEZ
}

Departments of Biostatistics and Physiology

CARL P. SIMON

Departments of Mathematics, Economics, and Public Policy

JAMES KOOPMAN

Department of Epidemiology

The University of Michigan, Ann Arbor, Michigan

LISA SATTENSPIEL

Department of Anthropology,

University of Missouri, Columbia, Missouri

AND

TIMOTHY PERRY

Departments of Physiology and Internal Medicine,

The University of Michigan, Ann Arbor, Michigan

Received 26 March 1988; revised 5 August 1988

\begin{abstract}
A compartmental model is presented for the spread of HIV in a homosexual population divided into subgroups by degree of sexual activity. The model includes constant recruitment rates for the susceptibles in the subgroups. It incorporates the long infectious period of HIV-infected individuals and allows one to vary infectiousness over the infectious period. A new pattern of mixing, termed preferred mixing, is defined, in which a fraction of a group's contacts can be reserved for within-group contacts, the remainder being subject to proportional mixing. The fraction reserved may differ among groups. In addition, the classic definition of reproductive number is generalized to show that for heterogeneous populations in general the endemic threshold is $\beta D c_{Y}$, where $c_{Y}$ is the mean number of contacts per infective. The most important finding is that the pattern of contacts between the different groups has a major effect on the spread of HIV, an effect inadequately recognized or studied heretofore.
\end{abstract}




\section{INTRODUCTION}

The AIDS epidemic has stimulated a large amount of research on the structure and variability of the human immune deficiency virus (HIV-1) and on the natural history and epidemiology of AIDS. Recently, a growing effort in modeling the transmission of HIV has emerged. The situation is unique in that modeling disease spread has been introduced into the discussions on epidemiology early in the process. Insightful modeling should help inform control efforts and provide a logical basis for designing the most informative population studies.

We have started a series of model studies with the general goal of obtaining insight into the structure of the transmission process. Our primary aim in the work reported here is to examine the effects of different patterns of contact in a population that can be divided into subgroups by degree of sexual activity. We find that both the rates of spread in the groups and the steady-state levels of HIV in the groups depend strongly on the pattern of contacts between the groups.

\section{NATURAL HISTORY AND EPIDEMIOLOGY OF AIDS}

We review briefly the important features of the spread of the HIV virus and the development of AIDS.

AIDS is caused by a retrovirus [1, 2], HIV-1 (formerly HTLV-III or LAV), which infects T-helper cells. This virus was identified as the etiological agent of AIDS in 1983 by Barre-Sinoussi et al. [3] and by Gallo et al. [4]. A closely related virus, HIV-2, found in humans in West Africa, has also been claimed to cause AIDS [5]. Similai but less closely related viruses have been found in T-cell leukemia (HTLV-1) and hairy cell leukemia (HTLV-2) in humans [6]; in terms of nucleotide sequence, HIV-1 is more closely related to visna virus [6]. There is also a related simian virus, SIV (STLV-3), which has caused problems as a contaminant in studies of human T-lymphotropic viruses.

The course of infection and progression to AIDS is highly variable. Immediately after infection there may be an acute illness with fever, myalgia, diarrhea, and lymphadenopathy that has been likened to a bout of infectious mononucleosis [7]; acute encephalitis has also been described at this stage [8]. This acute phase may correspond with rising amounts of free virus in the blood. There is then a fall in free virus as antibodies appear. This appearance of antibodies has been reported to occur in 19-56 days [7] or longer [9, 10]; in chimpanzees inoculated with the virus, antibodies appeared in 3 months [11]. The virus titer remains low for a variable period of years, but eventually the T-helper cell count begins to fall, virus levels rise, and antibodies to the viral core protein fall [12]. When the T-helper cell count falls below about $50 \%$ of the normal level, the opportunistic infections that are signs of the 
immune deficiency begin to appear. In addition, the virus eventually invades the brain, giving sign of dementia [8, 13-15]; it has been shown that HIV-1 inhibits neuronal responses to neurotropic factors in tissue culture [16].

The period from infection to appearance of first symptoms has been estimated to have a mean of 4.5 to 8 or more years [17-21]. Lui et al. [17, 18] fitted incidence data with a Weibull distribution and obtained a mean of 4.5 years ( $90 \%$ confidence interval: $2.6-14.2$ years) for cases infected by transfusion and 7.8 years (90\% confidence interval: $4.2-15$ years) for a cohort of homosexual and bisexual men. Medley et al. [20] use both Weibull and gamma distributions for the transfusion cases and obtain equally good fits; they report means of 2 years for children under $4,8.23$ years for those in the 5-59 age group, and 5.5 years for patients 60 years or older. Two problems have hampered accurate estimation of incubation times. For one, we have data only from the early phase of the epidemic of AIDS, so the data on distribution of incubation times are severely right-truncated [22, 23]. That problem is compounded by a distribution of reporting lags that makes the most recent data the least reliable because of underreporting. It is clear that as more data have accumulated the estimated incubation times have increased [22].

What fraction of those infected with HIV will eventually come down with AIDS? That too is uncertain, but the fraction has increased steadily as the data have accumulated. It may be that practically all will eventually succumb [18, 22, 24, 25]. Information on the nature of the virus is not encouraging. For the retroviruses, DNA transcripts of the viral RNA are incorporated into the genome of the host cell and may remain dormant for long periods. Stimulation of the cell by specific compounds may then activate synthesis of virus particles. It has been shown in tissue culture lines carrying HIV-1 that mitogens increase the replication of HIV-1 [26, 27]. In addition, the nucleotide sequence of HIV-1 is similar to that of visna virus [6], a lentivirus that causes progressive meningoencephalitis in sheep. As a group, the lentiviruses cause slow progressive diseases with high eventual fatality rates; the case fatality rate for visna virus is up to $100 \%$ [22]. This ancillary information, along with the progressively increasing estimates of mortality and incubation period, suggests the possibility of an eventual mortality pushing $100 \%$ with a long mean incubation time and an incubation time distribution that has a long tail. On the other hand, there is evidence that progression to AIDS may differ for individuals carrying different alleles of the group-specific component Gc [28].

HIV is transmitted by transfusion of infected blood, by sharing of needles by drug users, and by sexual intercourse. The first cases of AIDS were reported in 1981 [29], although there is now evidence that there may have been sporadic, unrecognized cases as far back as the 1960s. The early cases in the United States were in homosexual men and IV drug users [30-32], but 
heterosexual transmission has also been reported [33-36]. The combination of drug use and prostitution could play an important role in spreading HIV to heterosexual groups [29], but a study in New York City shows that this combination has not yet made a large contribution there [37]. The transmissibility per sexual act appears to be low but has been inferred to be much higher for anal receptive intercourse than for any other type of sexual contact [38-42]. However, accurate data on sexual contacts are difficult to obtain, and those available are for relationships that involve a variable, and often uncertain, number of contacts; so the estimates for transmissibility are subject to a number of uncertainties.

Because of the long incubation period before AIDS develops, there is now a large pool of HIV-infected individuals, much larger than the number of reported cases of AIDS, and there is reason to believe that most of these will come down with AIDS. However, the incidences of urethral and rectal gonorrhea in homosexual males have been falling steadily since 1981, suggesting that behavioral changes in some of the high risk groups have decreased contact rates and/or the probability of transmission per act [43].

\section{MODELING APPROACHES}

\section{GENERAL DISEASES}

An extensive literature on the modeling of epidemics has developed over the last two decades. Most of this literature has dealt with stationary populations characterized by fractions $S$ of susceptible individuals, $I$ of infected individuals, and $R$ of recovered and immune individuals who are still involved in the interactions that lead to transmission of the disease. Some models include a latent group $E$ of individuals who have been infected but are not yet infective. A now relatively standard terminology classifies models as SI, SIS, SIR, SIRS, and SEIRS, depending on what happens to infected individuals. Thus, an SI model applies to diseases in which infected individuals do not recover to return to the population. In an SIS model an infected person recovers and is again susceptible, whereas in an SIR model an infected person becomes immune on recovery. For reviews, see [44]-[50]. Appendix A contains the general equations for SIS and SIRS models and a description of the major results known about these models.

Of relevance for the work on AIDS is the modeling of contacts between individuals in homogeneous and heterogeneous populations. The most commonly used assumption has been that contacts are random; in a homogeneous population this assumption gives "homogeneous mixing." For a heterogeneous population divided into groups that have different contact rates, random mixing on the basis of activity gives "proportional mixing" [51-53]. Anderson and May [54] have looked at age-related heterogeneity in transmission of infection. May and Anderson [55] have used homogeneous 
mixing within groups and different uniform mixing rates between groups. Hethcote and Van Ark [53] have pointed out that the way in which May and Anderson defined their transmission parameter implies that the contact rates they use are proportional to group sizes. Travis and Lenhart [56] expanded the model of May and Anderson to include variable mixing rates between groups. Sattenspiel [57] and Sattenspiel and Simon [58] have used a migration matrix approach to specify the probabilities of contact between subgroups in a heterogeneous population in modeling an epidemic of hepatitis A. Ideally, one should estimate the contact rates both within and between groups. This was done for a model of influenza A [59]. The data needed to estimate contact rates are difficult to obtain. However, methods for estimating some transmission parameters from data are available [60-62].

A fair amount of work on modeling one sexually transmitted disease, gonorrhea, is available $[63,64]$. That work has stressed the importance of a highly active, promiscuous core group in spreading the disease.

\section{AIDS MODELING}

The number of efforts to model AIDS has grown rapidly. Pickering et al. [43] used a fínite difference model for a homosexual population in which they assumed homogeneous mixing. They included changes in contact rates with time that were estimated from the changes in incidence of rectal and urethral gonorrhea. Knox [65] reported on equilibria and the dynamics of some finite state models of the SIS and mixed SIS and SIR type. For multigroup networks, he introduced the contact matrix, which gives the contact rates between different groups. For the parameter values he chose, his calculations predicted that the prevalence of AIDS in promiscuous homosexuals would reach equilibrium in approximately 13 years, whereas the prevalence in nonpromiscuous heterosexuals would not reach equilibrium in 50 years. Kiesling et al. [66] also divide the population into groups based on sexual behavior.

May and Anderson [67] and Anderson et al. [68] used proportional mixing in modeling the spread of AIDS in groups of homosexual males that have different rates of contact with new partners. In a subsequent paper, Anderson et al. [69] report that their model is very sensitive to changes in values of the epidemiologic parameters and so take a pessimistic view of the possibility of predicting the size of the AIDS epidemic.

A number of models have been presented at meetings and have not yet appeared in journals. At the Los Alamos Conference on Nonlinearity in Medicine and Biology, Hyman and Stanley [70] reported their results with partial differential equation models of spread of HIV. The partial derivatives in their model arise from their treatment of risk as a continuous parameter. They show, as do we, that the degree of mixing between activity groups plays a major role in the spread of HIV into low-activity groups. At the same 
meeting Dietz [71] reported on a model of spread in the heterosexual population that includes some of the effects of pair formation. Hethcote's model [72] of HIV transmission emphasizes numerical simulations of the spread of the virus in major metropolitan areas, such as New York and San Francisco. It allows for 14 groups, including homosexuals, bisexuals, heterosexuals, and drug users, using proportional mixing for transmission in the homosexual community. Castillo-Chavez et al. [73] study a model that emphasizes the use of general survival functions to model the delays that occur in the transmission process and the use of contact parameters that vary with the size of the total population.

We present a model of HIV spread in a homosexual population that incorporates what we believe are three major factors in HIV spread. One factor is the effect of the pattern of contacts between groups that differ in sexual activity. A second factor is the long period of infectiousness prior to the onset of AIDS, which we model by a series of compartments representing stages an individual must pass through from the time of infection to the onset of clinical disease. Anderson et al. [74] have also used a chain of compartments to model stages and to introduce time lags in the numerical computations for their work on the demographic effects of AIDS [75]. The use of such a compartmental model permits one to examine a third factor, the effect of variation in transmission probability over the stages of infectiousness. We examine the effects of these three factors on the dynamic and steady-state behavior of the model.

\section{MODEL OF SPREAD IN A HOMOSEXUAL POPULATION}

\section{ASSUMPTIONS AND NOTATION}

We assume a population divided into $n$ groups based on sexual activity, the number of contacts per unit time. In this first model there is no migration between groups, but they interact by way of sexual contacts. Figure 1 shows a compartmental model [76] of the $i$ th contact-rate group. For the $i$ th group, $X_{i}$ is the number of susceptibles, $Y_{i}$ is the total number of infecteds, and $Z_{i}$ is the number with AIDS. From first infection to the development of AIDS, the infected pass through a series of $m$ stages,

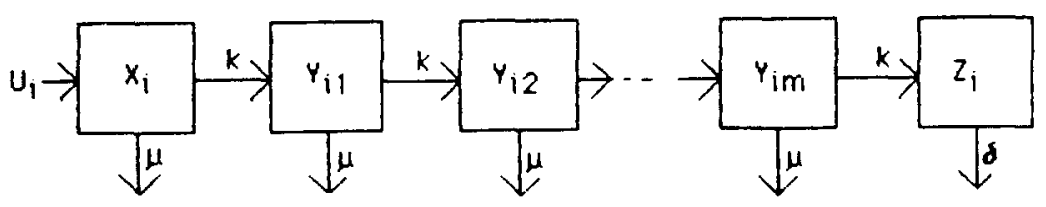

Fig. 1. Compartmental model for group $i$. 
$Y_{i 1}, \ldots, Y_{i m}$, with $Y_{i}=\sum_{r} Y_{i r}$. Those in $Z_{i}$ are presumed to be so ill with AIDS that they no longer take part in transmission. Thus the model consists of a set of $n$ SI models that interact by way of contacts between the susceptibles and infecteds of the submodels. We assume that the recruitment rate $U_{i}$ for the $i$ th group, the number of new susceptibles entering the group per unit of time, is constant.

We define the following parameters:

$c_{i}$, the number of persons contacted (scx acts) per person in group $i$ per unit time. This has dimensions $t^{-1}$. For very small populations the number of contacts per person per unit time might well be a function of the size of the population. We assume that we are not in that range and take a constant value for $c_{i}$.

$\rho_{i j}$, the proportion of the contacts of a person in group $i$ made with persons in group $j$; it is dimensionless, and $\sum_{j} \rho_{i j}=1$. The matrix $\mathbf{P}=\left[\left(\rho_{i j}\right)\right]$ is the mixing matrix or contact fraction matrix.

$\beta_{i j r}$, the transmission fraction. The fraction of contacts between a susceptible person in group $i$ and an infected person in $Y_{j r}$ that transmits the virus; dimensionless. In this paper we look only at $\beta_{i j r}$ that vary with $r$, the stage of the infection, but do not depend on $i$ and $j$.

$k$, the fractional transfer rate from $Y_{i r}$ to $Y_{i, r+1}$; dimension $t^{-1}$.

$\mu$, the fractional rate at which members transfer out of the groups for all reasons other than the development of AIDS. We call it the competing mortality rate, assumed constant for $X_{i}$ and $Y_{i}$ and assumed small relative to $k$.

$\delta$, the mortality rate for those with AIDS; dimension $t^{-1}$. This need not be used in the model $(\delta=0)$; if it is not used, $Z_{i}$ represents the total number who have developed AIDS, living and dead. We do not model the progression of the disease.

Note that the probability of transmission and the contact rates are of ten combined into one parameter. We carry them as distinct parameters because eventually we want to estimate them as such and we believe that various control measures may affect them independently.

\section{THE EQUATIONS}

With the parameters so defined, the rate at which susceptibles in group $i$ are infected by contact with members of $Y_{j}$ must be

$$
c_{i} X_{i} \rho_{i j} \beta_{i j r} \frac{Y_{j r}}{X_{j}+Y_{j}} .
$$

In this expression, $c_{i} X_{i}$ is the total number of contacts of $X_{i}$ per unit time, $\rho_{i j}$ gives the fraction of these contacts that are with group $j, Y_{j r} /\left(X_{j}+Y_{j}\right)$ is 
the probability that the contact with group $j$ is with a person in the $r$ th infectious subgroup, and $\beta_{i j r}$ is the fraction of those contacts that result in transmission. Thus the equation for the rate of change of $X_{i}$ is given by

$$
\dot{X}_{i}=-c_{i} X_{i} \sum_{j=1}^{n} \rho_{i j} \sum_{r=1}^{m} \beta_{i j r} \frac{Y_{j r}}{X_{j}+Y_{j}}-\mu X_{i}+U_{i}
$$

The equations for $Y_{i 1}, \ldots, Y_{i m}$ and $Z_{i}$ are then

$$
\begin{aligned}
& \dot{Y}_{i 1}=c_{i} X_{i} \sum_{j} \rho_{i j} \sum_{r} \beta_{i j r} \frac{Y_{j r}}{X_{j}+Y_{j}}-(k+\mu) Y_{i 1} \\
& \dot{Y}_{i r}=k Y_{i, r-1}-(k+\mu) Y_{i r}, \quad r=2, \ldots, m \\
& \dot{Z}_{i}=k Y_{i m}-\delta Z_{i} .
\end{aligned}
$$

This model differs in important ways from models frequently used in epidemiological modeling:

(1) With some exceptions [68, 74], the models commonly used are for a system of constant population size in which births equal deaths. We look at a population with constant rates of recruitment into the different groups. In the absence of AIDS and competing deaths, such populations would grow at constant rates. In the absence of HIV and with $\mu>0$, each population comes to a constant equilibrium population. We start with such equilibrium populations, introduce HIV, and follow the dynamics, seeking the conditions that give endemic steady states.

(2) The model explicitly includes a sequence of stages of infection that may differ in infectiousness, a feature also used by Anderson et al. [74, 75].

(3) We introduce a new type of mixing, preferred mixing, in which a fraction of each group's contacts can be reserved for intragroup contacts, a different fraction for each group.

An important consequence is that the rates of change of the susceptibles are not quadratic in susceptibles and infectives but are more complicated nonlinear, rational functions of the susceptibles and infectives, as illustrated by Equations (1) and (2).

\section{MODELING THE STAGES OF INFECTION}

The subgroups $Y_{i 1}, \ldots, Y_{i m}$ give the progression through the stages of infection up to the development of AIDS. If $\mu=0$, the density function of incubation times for a chain of $m$ compartments with uniform fractional transfer coefficient $k$ is the gamma distribution, Equation (5), with integer $m$ [76]:

$$
f(t)=\frac{k^{m} t^{m-1} e^{-k t}}{(m-1) !}
$$


This distribution has mode $(m-1) / k$, mean $m / k$, and variance $m / k^{2}$. For $\mu>0$ and for unit impulsive input into $Y_{i 1}$ at $t=0$, the amount leaving $Y_{i m}$ at $t$ is

$$
f(t)=\frac{k^{m} t^{m-1} e^{-(\mu+k) t}}{(m-1) !}
$$

The amount leaving $Y_{i m}$ is the fraction $k^{m} /(k+\mu)^{m}$ of the amount injected; the normalized density function for those that exit $Y_{i m}$ is Equation (5) with $(\mu+k)$ replacing $k$.

It is also possible to model the distribution of transit times through the stages of infection with a series of compartments that have different fractional transfer coefficients. That option provides more freedom in shaping the density function of transit times, but at the expense of an increase in algebraic complexity in the analysis of the endemic steady states.

\section{MIXING AND THE MIXING MATRIX}

Mixing between groups is specified by the contact fraction matrix, $\left(\rho_{i j}\right)$, which we also call the mixing matrix. The elements $\rho_{i j}$ give the proportion of the contacts of an individual in group $i$ that are made with individuals in group $j$. This matrix was used by Nold [51] in defining proportional mixing and corresponds to the migration matrix of Sattenspiel [57] and Sattenspiel and Simon [58]. Note that Knox [65] used a contact matrix rather than a contact fraction matrix.

\section{CONSTRAINTS}

One is not free to choose the elements of the mixing matrix. By its definition it is subject to two constraints. First, since the elements are proportions, the sum of each row must be $1: \sum_{j} \rho_{i j}=1$. In addition, there is a symmetry constraint. The number of contacts per unit time of group $i$ with $j$ must equal the number of contacts of $j$ with $i$, giving

$$
c_{i}\left(X_{i}+Y_{i}\right) \rho_{i j}=c_{j}\left(X_{j}+Y_{j}\right) \rho_{j i} \text {. }
$$

Only mixing matrices that satisfy these constraints are admissible. We shall examine the model results for three types of mixing: restricted, proportional, and preferred mixing.

\section{RESTRICTED MIXING}

In restricted mixing all contacts are restricted to within-group contacts. Consequently, $\rho_{i i}=1$ and $\mathbf{P}$ is the identity matrix. This matrix trivially satisfies the constraints. 
PROPORTIONAL MIXING

An assumption frequently used in epidemic models involving heterogeneous populations is the proportional mixing assumption $[51,53]$. In proportional mixing, the fraction of the contacts of group $i$ with group $j$ is equal to the fraction of total contacts made by the population that are due to group $j$. The total number of contacts by all groups is given by the equation

$$
C=\sum_{j} c_{j}\left(X_{j}+Y_{j}\right)
$$

Therefore, the $\rho_{i j}$ for proportional mixing are given by

$$
\rho_{i j}=c_{j} \frac{X_{j}+Y_{j}}{C}
$$

for all $i, j$. Consequently, $\mathbf{P}$ is a matrix all of whose rows are the same.

\section{PREFERRED MIXING}

In her work on heterogeneity in disease transmission, Nold [51] compared transmission for isolated groups (restricted mixing) and for groups with proportional mixing. She also looked at the case in which the mixing matrix was a linear combination of restricted and proportional mixing. Such mixing was also used by Hethcote et al. [77] in work on gonorrhea modeling and by Hethcote and Van Ark [53]. This approach is equivalent to reserving a fraction of the contacts of each group for within-group contacts, the same fraction for all groups. We have generalized that type of mixing so that arbitrary and different fractions of each group's contacts are reserved for within-group contacts; the nonreserved contacts are subject to proportional mixing. We have named this preferred mixing.

For preferred mixing, the elements $\rho_{i i}$ and $\rho_{i j}$ are given by

$$
\rho_{i i}=\rho_{i}+\left(1-\rho_{i}\right) \frac{c_{i}\left(1-\rho_{i}\right)\left(X_{i}+Y_{i}\right)}{\sum_{k} c_{k}\left(1-\rho_{k}\right)\left(X_{k}+Y_{k}\right)}
$$

and

$$
\rho_{i j}=\left(1-\rho_{i}\right) \frac{c_{j}\left(1-\rho_{i}\right)\left(X_{j}+Y_{j}\right)}{\sum_{k} c_{k}\left(1-\rho_{k}\right)\left(X_{k}+Y_{k}\right)}, \quad j \neq i
$$

In equations $(10)$ and $(11), \rho_{i}$ is the fraction of group $i$ 's contacts reserved for contacts within the group. Note that " $\rho_{i}=0$ for all $i$ " is full proportional mixing, while " $\rho_{i}=1$ for all $i$ " is restricted mixing, and " $0<\rho_{i}<1$ but all $\rho_{i}$ 
the same" is the case examined by Nold. Preferred mixing satisfies the constraints on the $\rho_{i j}$.

We note that with their model of age-structured contact rates, May et al. $[78,79]$ compare the effects of mixing restricted to people of the same age with those of mixing in which there is a constant probability of mixing with people of all ages. This is equivalent to comparing the effects of restricted mixing and proportional mixing in a finite state model.

\section{MATHEMATICAL PROPERTIES OF SYSTEM (1)-(4).}

As discussed above, the system of Equations (1)-(4) that we use to model the spread of AIDS differs in three significant ways from the usual SI, SIS, SIR, and SIRS systems of mathematical epidemiology. First, they include death from the disease and consequently are rational expressions of $X_{i}$ and $Y_{j}$, not simply quadratic expressions. Second, they include multiple stages of the disease; and finally, they allow for variable contact rates in a number of ways. In Appendix A, we write out a typical SIS or SIRS system and formally state a theorem that summarizes the current state of the theory for such equations. In summary, such systems have a compact, convex invariant domain $C$, and there is always a no-disease stationary solution. Furthermore, there is a function of the parameters $f(\lambda)$ and a threshold $b$ with the property that when $f(\lambda)<b$, the no-disease equilibrium is unique and globally asymptotically stable. When $f(\lambda)>b$, there is a unique new equilibrium that arises in the interior of $C$. This new endemic equilibrium is at least locally asymptotically stable and probably globally so.

How much of this general theory carries over to our system (1)-(4)? The following theorem summarizes our theoretical results for this system.

\section{THEOREM 1}

Consider the system (1)-(4) with $\mu>0$ and with restricted, proportional, or preferred mixing. Then:

(i) There is a compact (i.e., closed and bounded), convex invariant set $C$ in the nonnegative orthant, so that all solutions that start in $C$ stay in $C$ for all $t>0$.

(ii) The no-disease steady state $X_{i}=U_{i} / \mu, Y_{i}=0$ for all $i$, is always $a$ stationary solution of (1)-(4).

(iii) There is a function $f$ of the parameters and $a$ constant $b$ with the property that when $f(\lambda)<b$ the no-disease equilibrium is the only stationary solution, and when $f(\lambda)>b$ the no-disease equilibrium and an interior, endemic equilibrium are the only stationary solutions of (1)-(4).

(iv) For the case of restricted mixing, when $f(\lambda)<b$ the no-disease equilibrium is globally asymptotically stable, and when $f(\lambda)>b$ the no-disease equilibrium becomes unstable and the endemic equilibrium is locally asymptotically stable. 


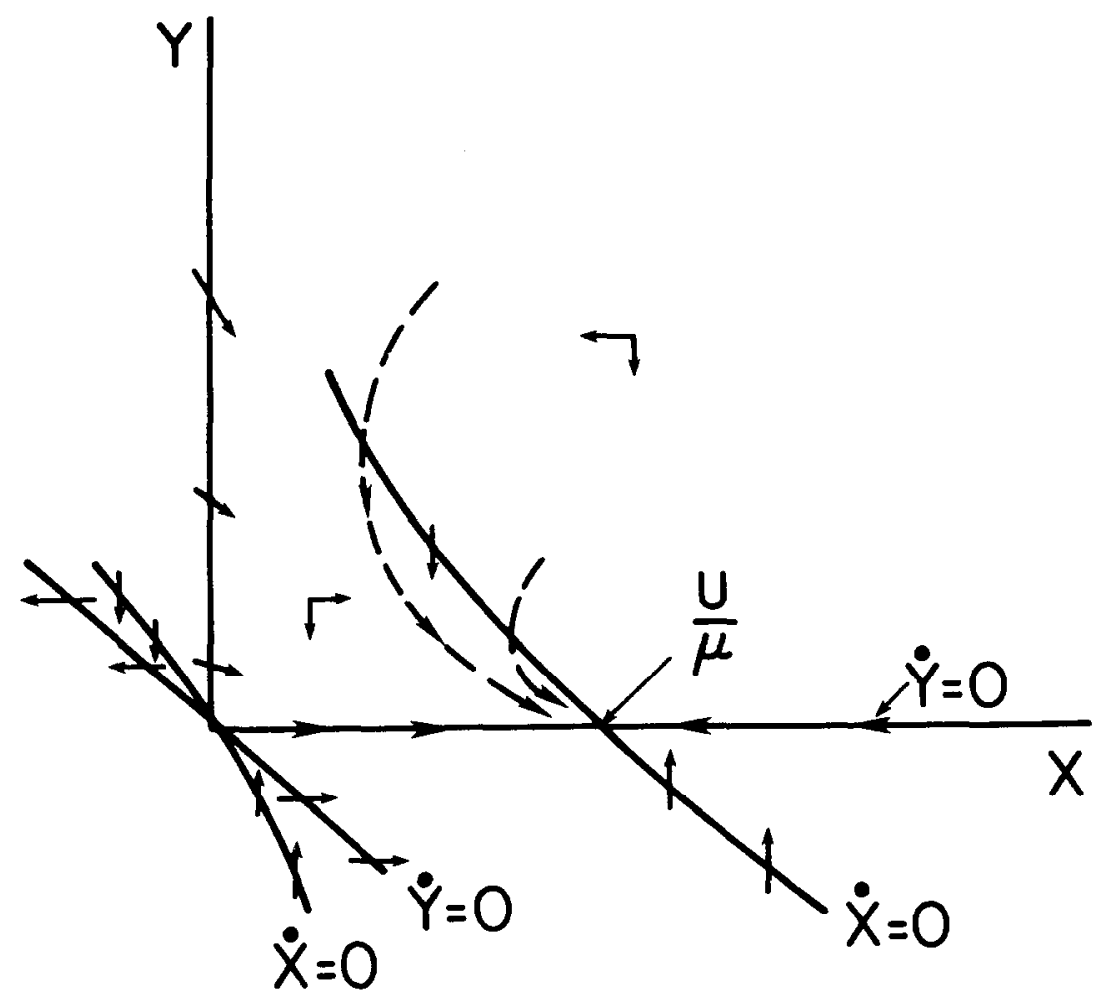

FIG. 2. Phase diagram for simplified system with one group and one stage for $\mu>0$ and $\lambda-(k+\mu)$ negative.

For $\mu=0$, the no-disease equilibrium occurs at $X_{i}$ "equal to infinity" and $Y_{i}=0$. In this case, there is no compact invariant set.

The proofs of parts i, ii, and iv of Theorem 1 are presented in Appendix A. The existence of the threshold and the endemic equilibrium is computed in Section 7 for $\mu=0$ and in Appendix $C$ for $\mu>0$. Section 8 describes how these thresholds and endemic steady states depend on the parameters of the model.

To further the reader's intuition about system (1)-(4), we present in Figures 2 and 3 the planar phase diagrams for a simplified version of (1)-(4) with one subgroup, with deaths, and with only one stage. The one subgroup condition is equivalent to restricted mixing. The one-stage hypothesis is made so that our system will be planar. The equations for the spread of the 


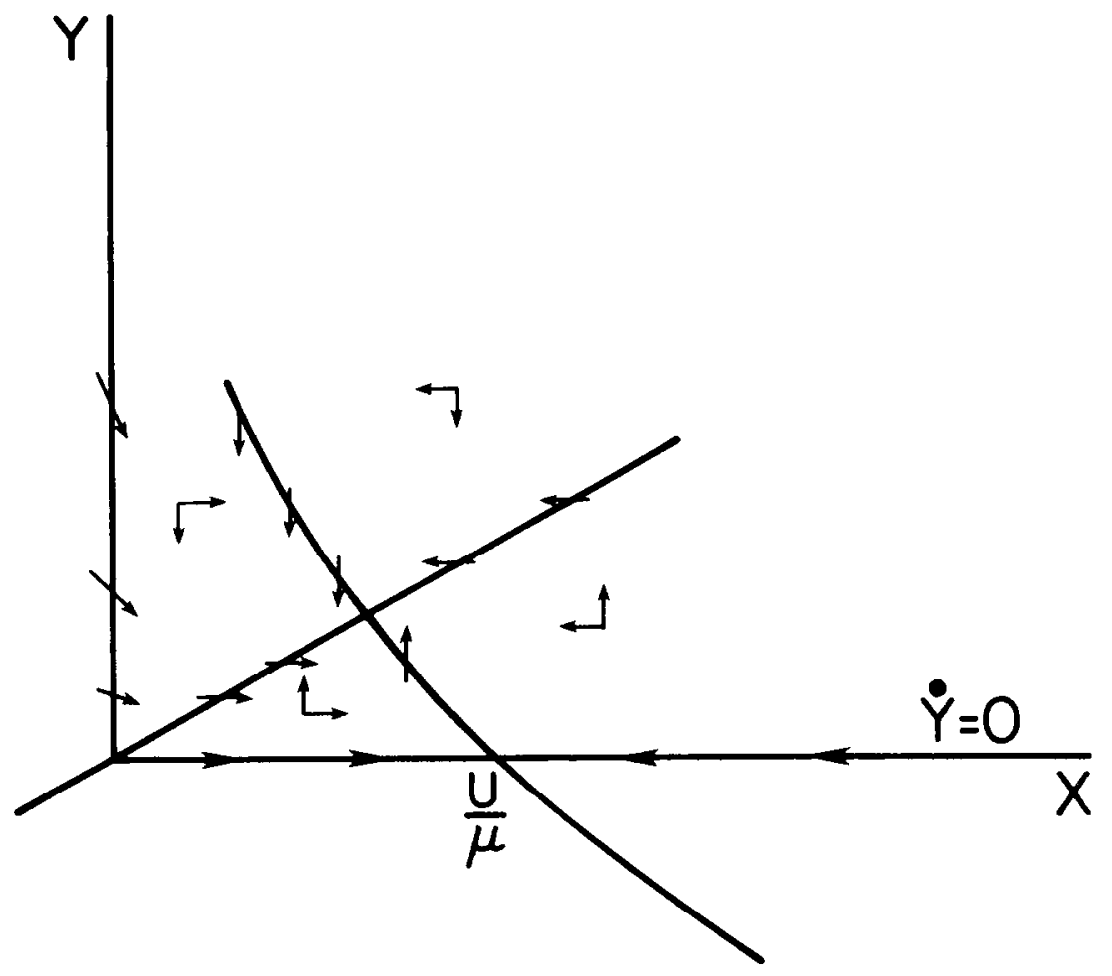

FIG. 3. Phase diagram for simplified system with $\lambda-(k+\mu)$ positive.

disease in this case are

$$
\begin{aligned}
\dot{X} & =-\frac{\lambda X Y}{X+Y}-\mu X+U, \quad \lambda=\beta c \\
\dot{Y} & =\frac{\lambda X Y}{X+Y}-(k+\mu) Y, \\
\dot{Z} & =k Y-\delta Z
\end{aligned}
$$

Since the first two equations are independent of $Z$, we can work with just these two, without loss of generality. May et al. [78, 79] give the phase plane diagrams for the same system of equations in terms of $Y$ and $N=X+Y$.

In Figures 2 and 3, the threshold condition is whether or not $\lambda-(k+\mu)$ is negative or positive. When it is negative, the $\{\dot{Y}=0\}$ isocline $\gamma_{1}$ lies in the second and fourth quadrants and crosses the $\{\dot{X}=0\}$ isocline $\gamma_{2}$ in the fourth quadrant, as illustrated in Figure 2. In this case, the no-disease 
equilibrium is the only stationary solution, and it is globally asymptotically stable with $V(X, Y)=Y$ a Lyapunov function for the system. When $\lambda-$ $(k+\mu)$ is positive, the isocline $\gamma_{1}$ lies in the first and third quadrants and crosses isocline $\gamma_{2}$ in the positive quadrant at the endemic equilibrium, as pictured in Figure 3. Our analysis of this situation in Appendix A shows that the endemic equilibrium is globally asymptotically stable in this case. Note that the endemic equilibrium arises when the $Y$ coordinate of the crossing of $\gamma_{1}$ and $\gamma_{2}$ becomes positive and in this case the stability of the system transfers from the no-disease equilibrium to the endemic one.

Figures A1 and A2 in Appendix A study the same phenomenon for the case $\mu=0$.

\section{ENDEMIC STEADY STATES AND THRESHOLDS}

In this section we derive the steady-state results and thresholds for $\mu=0$. The results for $\mu>0$ are more realistic in that the population groups come to stationary states even if some of the groups are below their infectious thresholds. However, the algebra for $\mu>0$ becomes so cumbersome that it is difficult to see the forest for the trees. Moreover, the pattern of the derivation is the same for $\mu>0$ as for $\mu=0$. Therefore, we present the results for $\mu=0$ as a template for the derivation for $\mu>0$. The results of the calculations in this section and in Appendix $\mathrm{C}$ are summarized in Tables 1 and 2. The interpretations of the thresholds are presented in Section 9.

\section{THE STEADY STATES FOR $\mu=0$}

There is a steady state in the $Y_{j}$ 's when there are no infected individuals in the population; $Y_{j}=0$ for all $j$. But then $\dot{X}_{i}=U_{i}$ and the susceptible populations grow without bound. We look for steady states at which $\dot{X}_{i}$ and $\dot{Y}_{i}$ are both zero for all $i$. We write $X_{i}^{s}$ and $Y_{i}^{s}$ for the values of $X_{i}$ and $Y_{i}$ at the stationary solutions. For $\mu=0$, Equations (1)-(3) reduce to (13)-(16) at the steady state.

$$
\begin{gathered}
U_{i}=c_{i} X_{i}^{s} \sum_{j=1}^{n} \rho_{i j} \sum_{r=1}^{m} \beta_{i j r} \frac{Y_{j r}^{s}}{X_{j}^{s}+Y_{j}^{s}} \\
Y_{i 1}^{s}=\frac{1}{k} c_{i} X_{i}^{s} \sum_{j} \rho_{i j} \sum_{r} \beta_{i j r} \frac{Y_{j r}^{s}}{X_{j}^{s}+Y_{j}^{s}}=\frac{U_{i}}{k} \\
Y_{i r}^{s}=Y_{i, r-1}^{s}=\frac{U_{i}}{k}, \quad \text { for } r=2, \ldots, m .
\end{gathered}
$$

From (15) it follows that

$$
Y_{i}^{s}=\sum_{r=1}^{m} Y_{i r}^{s}=\frac{m U_{i}}{k}
$$


At the endemic steady state, the size of the infected population is a rather simple expression (16), which is independent of the type of mixing under consideration. This fact makes the calculations for $\mu=0$ much simpler than the corresponding calculations for $\mu>0$.

THE STEADY STATES DEPEND ON THE MEAN VALUES OF $\beta_{i, r}$

In this paper we will be concerned with transmission fractions that depend only on the stage of HIV infection, so we can write $\beta_{i j r}=\beta_{r}$. From (15) and (16), $Y_{j r}^{s}=Y_{j}^{s} / m$. Substituting into (13) and rearranging gives

$$
U_{i}=c_{i} X_{i}^{s} \sum_{j} \rho_{i j} \frac{Y_{j}^{s}}{X_{j}^{s}+Y_{j}^{s}} \sum_{r=1}^{m} \frac{\beta_{r}}{m}=c_{i} X_{i}^{s} \bar{\beta} \sum_{j} \rho_{i j} \frac{Y_{j}^{s}}{X_{j}^{s}+Y_{j}^{s}} .
$$

Note that the summation over $r$ in (17) is the mean value $\bar{\beta}$ over the stages in a group.

RESTRICTED MIXING

For restricted mixing, Equation (17) simplifies to

$$
U_{i}=c_{i} \bar{\beta} X_{i}^{s} \frac{Y_{i}^{s}}{X_{i}^{s}+Y_{i}^{s}}
$$

Multiplying (18) by $\left(X_{i}^{s}+Y_{i}^{s}\right)$, substituting $Y_{i}^{s}=(m / k) U_{i}$, and solving for $X_{i}^{s}$ gives

$$
X_{i}^{s}=\frac{(m / k) U_{i}}{\bar{\beta} c_{i}(m / k)-1}
$$

Since $Y_{i}^{s}$ is $(m / k) U_{i}$, the steady-state infectious fraction for group $i$ is then

$$
\frac{Y_{i}^{s}}{X_{i}^{s}+Y_{i}^{s}}=\frac{\bar{\beta} c_{i} m / k-1}{\bar{\beta} c_{i} m / k}
$$

The numerator of (20) [the denominator of (19)] must be greater than zero for there to be an endemic steady state. Note that $\bar{\beta} c_{i} m / k$ is the initial reproductive rate $R_{0}[46,53,55,80]$ and must be greater than 1 for an epidemic to start. This condition also defines an endemic threshold in $\bar{\beta}$ for group $i$; if we think of $c_{i}$ and $\mathrm{m} / \mathrm{k}$ as being determined, then $\bar{\beta}$ must be greater than

$$
\bar{\beta}_{T, i} \equiv \frac{1}{c: m / k}
$$


for the existence of an endemic state with infected fraction greater than zero. With this notation, the steady-state infected fraction can be written as

$$
\frac{Y_{i}^{s}}{X_{i}^{s}+Y_{i}^{s}}=1-\frac{\bar{\beta}_{T, i}}{\bar{\beta}}
$$

PROPORTIONAL MIXING

We turn next to proportional mixing where $\rho_{i j}$ is given by expression (9). Substituting Equation (9) for $\rho_{i j}$ into Equation (17) gives, for the steady state,

$$
U_{i}=\bar{\beta} c_{i} X_{i}^{s} \frac{\sum c_{j} Y_{j}^{s}}{\sum c_{j} X_{j}^{s}+\sum c_{j} Y_{j}^{s}}
$$

Sum Equation (22) over $i$ and solve for $\sum_{j} c_{j} X_{j}^{s}$ :

$$
\sum_{j} c_{j} X_{j}^{s}=\frac{(m / k)\left(\sum c_{j} U_{j} / \Sigma U_{j}\right) \Sigma U_{j}}{(\bar{\beta} m / k)\left(\sum c_{j} U_{j} / \Sigma U_{j}\right)-1} .
$$

It is convenient at this point to introduce an average of the contacts of the infected individuals in the population:

$$
\bar{c}_{Y}=\frac{\sum_{j} c_{j} Y_{j}}{\sum_{j} Y_{j}} .
$$

Note that this average changes with time; at the steady state, $Y_{j}=U_{j}(m / k)$ and $\bar{c}_{Y}$ becomes $\bar{c}_{U}$ :

$$
\bar{c}_{U}=\frac{\sum c_{j} U_{j}}{\sum U_{j}}
$$

Substituting $\bar{c}_{U}$ into (23) yields

$$
\sum_{j} c_{j} X_{j}^{s}=\frac{(m / k) \bar{c}_{U} \Sigma U_{j}}{\bar{\beta} \bar{c}_{U}(m / k)-1} .
$$

We define $K_{i Y}$ by the equation

$$
K_{i Y}=\sum_{j} \rho_{i j} \frac{Y_{j}}{X_{j}+Y_{j}}
$$

The number $K_{i Y}$ is the fraction of group $i$ 's contacts that are with infectious 
individuals. Using the $\rho_{i j}$ for proportional mixing from Equation (9), we find that $K_{i Y}$ is independent of $i$ :

$$
K_{i Y}=K_{Y}=\frac{\sum c_{j} Y_{j}}{\sum c_{j} X_{j}+\sum c_{j} Y_{j}}
$$

At the steady state, substitute (26) into (28) to obtain

$$
K_{Y}^{s}=\frac{\bar{\beta} \bar{c}_{U}(m / k)-1}{\bar{\beta} \bar{c}_{U}(m / k)}
$$

Now, substitute (29) into (22) and solve for $X_{i}^{s}$ :

$$
X_{i}^{s}=\frac{U_{i}}{\bar{\beta} c_{i} K_{Y}^{s}}=\frac{(m / k) \bar{c}_{U} U_{i}}{c_{i}\left(\bar{\beta} \bar{c}_{U}(m / k)-1\right)}
$$

The fraction infected in group $i$ at the steady state can now be written as

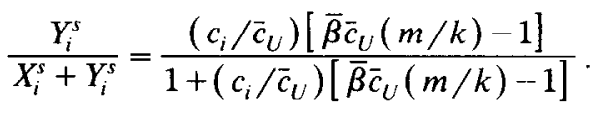

For an endemic state, the numerator of (31) [the denominator of (30)] must be greater than zero. This condition once more defines a threshold in transmission rates but one that is the same for all groups, that is, a population threshold:

$$
\bar{\beta}_{T}=\frac{1}{\bar{c}_{U}(m / k)}
$$

In this notation, we can write (31) as

$$
\frac{Y_{i}^{s}}{X_{i}^{s}+Y_{i}^{s}}=\frac{\left(c_{i} / \bar{c}_{U}\right)\left[\left(\bar{\beta} / \bar{\beta}_{T}\right)-1\right]}{1+\left(c_{i} / \bar{c}_{U}\right)\left[\left(\bar{\beta} / \bar{\beta}_{T}\right)-1\right]}
$$

\section{PREFERRED MIXING}

In the case of preferred mixing, plug the mixing fractions $\rho_{i j}$ from (10) and (11) into the steady-state equation (17) to obtain the equation for the steady-state value $X_{i}^{s}$ :

$$
U_{i}=\bar{\beta} c_{i} X_{i}^{s}\left(\frac{\rho_{i} Y_{i}^{s}}{X_{i}^{s}+Y_{i}^{s}}+\left(1-\rho_{i}\right) \frac{\sum c_{j}\left(1-\rho_{j}\right) Y_{j}^{s}}{\sum c_{i}\left(1-\rho_{i}\right) X_{i}^{s}+\sum c_{i}\left(1-\rho_{i}\right) Y_{i}^{s}}\right)
$$


In analogy with definition (27) in the proportional mixing case, we define $K_{Y}$ by

$$
K_{Y}=\frac{\sum c_{j}\left(1-\rho_{j}\right) Y_{j}}{\sum c_{j}\left(1-\rho_{j}\right) X_{j}+\sum c_{j}\left(1-\rho_{j}\right) Y_{j}} .
$$

Here $K_{Y}$ is the fraction of the contacts subject to proportional mixing that are with infectious individuals; $K_{Y}$ is the same for all groups. However, at the steady state $K_{Y}=K_{Y}^{s}$ we no longer have a simple expression for $K_{Y}^{s}$ corresponding to expression (29) for full proportional mixing.

We cannot find a closed solution for $X_{i}^{s}$ in this preferred mixing case, but substituting $K_{Y}^{s}$ from (34) into (33) and rearranging yields a quadratic equation for $X_{i}^{s}$ :

$$
\begin{aligned}
& \bar{\beta} c_{i}\left(1-\rho_{i}\right) K_{Y}^{s}\left(X_{i}^{s}\right)^{2}+\left\{\bar{\beta} c_{i}(m / k)\left[\left(1-\rho_{i}\right) K_{Y}^{s}+\rho_{i}\right]-1\right\} U_{i} X_{i}^{s}-(m / k) U_{i}^{2} \\
& \quad=0 .
\end{aligned}
$$

We use the quadratic formula to write the solution to the quadratic (35) as

$$
X_{i}^{s}=\frac{U_{i}\left(-P_{i}+\sqrt{P_{i}^{2}+Q_{i}}\right)}{2 \bar{\beta} c_{i}\left(1-\rho_{i}\right) K_{Y}^{s}}
$$

where

$$
\begin{aligned}
P_{i} & =\bar{\beta} c_{i}(m / k)\left(\left(1-\rho_{i}\right) K_{Y}^{s}+\rho_{i}\right)-1, \\
Q_{i} & =4 \bar{\beta} c_{i}(m / k)\left(1-\rho_{i}\right) K_{Y}^{s} .
\end{aligned}
$$

We have used the positive root in the quadratic formula to guarantee that $X_{i}^{s}$ is nonnegative. The coefficients in (36) depend on $K_{Y}^{s}$, which in turn depends on $X_{i}^{s}$. We have calculated solutions iteratively by first estimating $K_{Y}^{s}$, then calculating $X_{i}^{s}$ using (36), then calculating $K_{Y}^{s}$ using (34), and so on. This iteration procedure converges rapidly. In Appendix B, we show why this iteration works so well, and we prove that the endemic solution is unique. We also compute the threshold for endemicity:

$$
\tau \equiv \frac{\sum_{j} \max \left\{0,1-\bar{\beta} c_{j}(m / k) \rho_{j}\right\} U_{j}}{\bar{\beta}(m / k) \sum_{j} c_{j}\left(1-\rho_{j}\right) U_{j}} .
$$

When $\tau \leqslant 1$, there is a unique endemic equilibrium; when $\tau>1$, there is no endemic equilibrium. Furthermore, this condition reduces to our earlier threshold for the proportional mixing case, when all the $\rho_{i}$ 's equal 0 . As we discuss at the end of Appendix B, it is also consistent with the threshold conditions we derived for restricted mixing $\left(\rho_{i}=1\right.$ for all $i$ ).

The steady-state results are summarized in Tables 1 and 2 . 
TABLE 1

Steady State Results for Different Mixing Matrices for $\mu=0$

\begin{tabular}{|c|c|c|c|}
\hline & Restricted & Proportional & Preferred \\
\hline$X_{i}^{\mathrm{s}}$ & $\frac{(m / k) U_{i}}{\bar{\beta} c_{i}(m / k)-1}$ & $\frac{(m / k) U_{i}}{\left(c_{i} / \bar{c}_{U}\right)\left(\bar{\beta} \bar{c}_{U}(m / k)-1\right)}$ & $\frac{U_{i}\left(-P_{i}+\sqrt{P_{i}^{2}+Q_{i}}\right)}{2 \bar{\beta} c_{i}\left(1-\rho_{i}\right) K_{Y}^{x}}$ \\
\hline$Y_{i}^{3}$ & $U_{i}(m / k)$ & $U_{i}(m / k)$ & $U_{i}(m / k)$ \\
\hline End. Thr. ${ }^{a}$ & $\bar{\beta}_{i} c_{i} \frac{m}{k}>1$ & $\bar{\beta} \bar{c}_{U} \frac{m}{k}>1$ & $\frac{\bar{\beta}(m / k) \sum_{j} c_{j}\left(1-\rho_{j}\right) U_{j}}{\sum \max \left\{0,1-\bar{\beta} c_{j} \rho_{j}(m / k)\right\} U_{j}}>1$ \\
\hline $\bar{\beta}_{T, \imath}$ & $\frac{1}{c_{i} m / k}$ & $\frac{1}{\bar{c}_{u}(m / k)}$ & ${\frac{1}{\bar{c}_{U}(m / k)}}^{n}$ \\
\hline$\frac{Y_{i}^{s}}{X_{i}^{s}+Y_{i}^{s}}$ & $1-\overline{\boldsymbol{\beta}}_{T, i} / \overline{\boldsymbol{\beta}}$ & $\frac{\left(c_{i} / \bar{c}_{U}\right)\left[\left(\bar{\beta} / \bar{\beta}_{T}\right)-1\right]}{1+\left(c_{i} / \bar{c}_{U}\right)\left[\left(\bar{\beta} / \bar{\beta}_{T}\right)-1\right]}$ & $\frac{\frac{1}{2} Q_{i}}{\frac{1}{2} Q_{i}-P_{i}+\sqrt{P_{i}^{2}+Q_{i}}}$ \\
\hline
\end{tabular}

${ }^{a}$ End. Thr. $=$ endemicity threshold.

bAssumes $1-\bar{\beta} c_{i} \rho_{i}(m / k)>0$ for all $i$.

TABLE 2

Steady-State Results for Different Mixing Matrices for $\mu>0$

\begin{tabular}{lccc}
\hline & Restricted & Proportional & Preferred \\
\hline$X_{i}^{s}$ & $\frac{\theta_{\mu} U_{i}}{\bar{\beta}_{\mu} c_{i} \theta_{\mu}-\alpha^{m}}$ & $\frac{\theta_{\mu} U_{i}}{\mu \theta_{\mu}+\frac{c_{i}}{\bar{c}_{w}}\left(\bar{\beta}_{\mu} \bar{c}_{w} \theta_{\mu}-1\right)}$ & $\frac{A_{i} U_{i}}{K_{Y}^{s}+\mu A_{i}}$ \\
$Y_{i}^{s}$ & $\frac{c_{i} \bar{\beta}_{\mu}\left(\theta_{\mu}-1\right) \theta_{\mu} U_{i}}{c_{i} \bar{\beta}_{\mu}-\alpha^{m}}$ & $\frac{\theta_{\mu}\left(c_{i} / \bar{c}_{w}\right)\left(\bar{\beta}_{\mu} \theta_{\mu} \bar{c}_{w^{\prime}}-1\right) U_{i}}{\mu \theta_{\mu}+\left(c_{i} / \bar{c}_{w}\right)\left(\bar{\beta}_{\mu} \theta_{\mu} \bar{c}_{w}-1\right)}$ & $\frac{\theta_{\mu} U_{i} K_{Y}^{s}}{K_{Y}^{s}+\mu A_{i}}$ \\
End. Thr. ${ }^{a}$ & $\bar{\beta}_{\mu} \theta_{\mu} c_{i}>1$ & $\bar{\beta}_{\mu} \theta_{\mu} \bar{c}_{w}>1$ & See App. C \\
$\bar{\beta}_{\mu . T}$ & $\frac{1}{c_{i} \theta_{\mu}}$ & $\frac{1}{\bar{c}_{w} \theta_{\mu}}$ & See App. C \\
$\frac{Y_{i}^{s}}{X_{i}^{s}+Y_{i}^{s}}$ & $1-\bar{\beta}_{\mu, T} / \bar{\beta}_{\mu}$ & $\frac{\left(c_{i} / \bar{c}_{w}\right)\left[\left(\bar{\beta}_{\mu} / \bar{\beta}_{\mu, T}\right)-1\right]}{1+\left(c_{i} / \bar{c}_{w}\right)\left[\left(\bar{\beta}_{\mu} / \bar{\beta}_{\mu, T}\right)-1\right]}$ & $\frac{\theta_{\mu} K_{Y}^{s}}{A_{i}+\theta_{\mu} K_{Y}^{s}}$ \\
\hline
\end{tabular}

${ }^{\mathrm{a}}$ End. Thr. = endemicity threshold. 
In Table 1,

$$
\begin{aligned}
\bar{c}_{U} & =\frac{\sum c_{i} U_{i}}{\sum U_{i}}, \\
P_{i} & =\bar{\beta} c_{i}(m / k)\left[\left(1-\rho_{i}\right) K^{s}+\rho_{i}\right]-1, \\
Q_{i} & =4 \bar{\beta} c_{i}(m / k)\left(1-\rho_{i}\right) K^{s},
\end{aligned}
$$

and

$$
K_{Y}^{s}=\frac{\sum c_{j}\left(1-\rho_{j}\right) Y_{j}^{s}}{\sum c_{j}\left(1-\rho_{j}\right) X_{j}^{s}+\sum c_{j}\left(1-\rho_{j}\right) Y_{j}^{s}}
$$

In Table 2,

$$
\begin{aligned}
\alpha & =\frac{k}{k+\mu}, \quad \theta_{\mu}=\frac{1-\alpha^{m}}{\mu}, \\
\left(1-\alpha^{m}\right) & =\frac{m}{k}\left(\frac{k}{k+\mu}\right)^{m} \mu\left[1+g\left(\frac{\mu}{k}\right)\right], \\
K_{Y}^{s} & =\frac{\sum c_{j}\left(1-\rho_{j}\right) Y_{j}^{s}}{\sum c_{j}\left(1-\rho_{j}\right) X_{j}^{s}+\sum c_{j}\left(1-\rho_{j}\right) Y_{j}^{s}}, \\
P_{i} & =\bar{\beta}_{\mu} c_{i} \theta_{\mu}\left[\left(1-\rho_{i}\right) K_{Y}^{s}+\rho_{i}\right]-1, \\
Q_{i} & =4 \bar{\beta}_{\mu} c_{i} \theta_{\mu}\left(1-\rho_{i}\right) K_{Y}^{s} \\
A_{i} & =\frac{-P_{i}+\sqrt{P_{i}^{2}+Q_{i}}}{2 c_{i} \bar{\beta}_{\mu}\left(1-\rho_{i}\right)}
\end{aligned}
$$

and

$$
\bar{\beta}_{\mu}=\sum_{r=1}^{m} \frac{k^{r-1} /(k+\mu)^{r}}{\frac{1}{\mu}\left[1-\left(\frac{k}{k+\mu}\right)^{m}\right]} \beta_{i j r}
$$

\section{DEPENDENCE OF EQUILIBRIA ON THE PARAMETERS}

Since we have been able to compute the endemic equilibria and thresholds of endemicity in our rather complex dynamic model, the next natural step is to calculate how the parameters in the model affect the values of the equilibria and thresholds. The sensitivities of the steady states to changes in the parameters provide important information to guide educational efforts and control strategies. For restricted and proportional mixing, we need only compute the derivatives of the entries in the middle two columns of Tables 1 
and 2 to understand these sensitivities. However, for preferred mixing, this process is more difficult because we have only implicit equations for the equilibria. The calculations for preferred mixing are carried out in Appendix D.

We will treat each of the parameters

$$
c_{1}, \ldots, c_{n}, \bar{\beta}, \rho_{1}, \ldots, \rho_{n}, U_{1}, \ldots, U_{n}, m / k
$$

one at a time. We will prove our results for $\mu=0$. The corresponding results for $\mu>0$ either follow directly by the same method or hold automatically for $\mu$ small and positive because of the continuous dependence on the parameters. [The only results that do not carry over to $\mu>0$ are the zero derivatives of $Y_{i}^{s}$ with respect to most parameters, because of the very simple expression (39) for $Y_{i}^{s}$ in the $\mu=0$ case.]

The Rate of Sexual Activity $c_{i}$. For restricted mixing and preferred mixing,

$$
\frac{\partial X_{i}^{s}}{\partial c_{i}}<0 \quad \text { and } \quad \frac{\partial}{\partial c_{i}}\left(\frac{Y_{i}^{s}}{X_{i}^{s}+Y_{i}^{s}}\right)>0
$$

For proportional mixing,

$$
\frac{\partial X_{j}^{s}}{\partial c_{i}}<0 \quad \text { and } \quad \frac{\partial}{\partial c_{i}}\left(\frac{Y_{j}^{s}}{X_{j}^{s}+Y_{j}^{s}}\right)>0,
$$

for all $i, j$. As sexual activity in any group rises, the infected fraction in that group rises.

For preferred mixing, (38) holds for all $i$ and (39) holds for all groups $i$ whose infected fraction is less than average:

$$
\frac{Y_{i}^{s}}{X_{i}^{s}+Y_{i}^{s}}<K_{Y}^{s}
$$

Increasing the sexual activity of such low-activity groups increases the infected fractions of all groups. On the other hand, increasing the sexual activity of a very active or highly infected group could deplete the equilibrium population of that group so badly that there are fewer members of that group available for interactions with other groups. This scenario would actually decrease the infected fractions of the other groups.

The value of the threshold for subgroup $i$ falls as $c_{i}$ rises for restricted mixing and as any $c_{j}$ rises for proportional and preferred mixing. In other words, the higher the level of sexual activity in any group, the more likely it is that the system will reach an endemic equilibrium. 
The Transmission Fraction $\beta_{i j k}$. As we computed in Section 7 , the equilibria and thresholds depend only on the mean transmission fraction

$$
\bar{\beta}=\frac{1}{m} \sum_{r=1}^{m} \beta_{i j r}=\frac{1}{m} \sum_{r=1}^{m} \beta_{r}
$$

so we need only work with $\bar{\beta}$, which is itself an increasing function of the $\beta_{i j r}$. For all three types of mixing,

$$
\frac{\partial X_{i}^{s}}{\partial \bar{\beta}}<0 \quad \text { and } \quad \frac{\partial}{\partial \bar{\beta}}\left(\frac{Y_{i}^{s}}{X_{i}^{s}+Y_{i}^{s}}\right)>0
$$

As any probability of transmission increases, the infected fraction increases; furthermore, the threshold condition becomes easier to satisfy, so that it is more likely that an endemic equilibrium will be reached.

The Subgroup Recruitment Rate $U_{i}$. The results for the recruitment parameter $U_{i}$ depend very much on the type of mixing under study. For restricted mixing,

$$
\frac{\partial X_{i}^{s}}{\partial U_{i}}>0, \quad \frac{\partial Y_{i}^{s}}{\partial U_{i}}>0, \quad \text { and } \quad \frac{\partial}{\partial U_{i}}\left(\frac{Y_{i}^{s}}{X_{i}^{s}+Y_{i}^{s}}\right)=0
$$

the infected fraction is independent of the rate of recruitment, as is the value of the threshold. For proportional and preferred mixing, $\partial Y_{i}^{s} / \partial U_{i}$ is still the positive number $m / k$, but the effects on $X_{i}^{s}$ and on the infected fractions depend on the characteristics of the subgroup. For proportional mixing,

$$
\begin{aligned}
& \frac{\partial X_{i}}{\partial U_{i}}=\frac{m / k}{\frac{c_{i}}{\bar{c}_{U}}\left[\bar{\beta} \bar{c}_{U} \frac{m}{k}-1\right]}-\frac{U_{i}(m / k) \Sigma_{j}\left(c_{i}-c_{j}\right) U_{j}}{c_{i}\left(\sum_{j} U_{j}\right)^{2}\left[\bar{\beta} \bar{c}_{U} \frac{m}{k}-1\right]^{2}}, \\
& \frac{\partial X_{i}}{\partial U_{h}}=-\frac{U_{i}(m / k) \Sigma_{j}\left(c_{h}-c_{j}\right) U_{j}}{c_{i}\left(\sum_{j} U_{j}\right)^{2}\left[\bar{\beta} \bar{c}_{U} \frac{m}{k}-1\right]^{2}}, \quad h \neq i .
\end{aligned}
$$

The term in the square brackets in (41) is always positive for an endemic equilibrium by the threshold condition. If $\sum_{j}\left(c_{i}-c_{j}\right) U_{j}$ is negative-for example, if $c_{i}=\min _{j} c_{j}$-then $\partial X_{i}^{s} / \partial U_{i}$ is positive; for low-activity groups, increasing recruitment increases both $X_{i}^{s}$ and $Y_{i}^{s}$. However, if $\Sigma_{j}\left(c_{i}-c_{j}\right) U_{j}$ $>0$-for example, if $c_{i}=\max _{j} c_{j}$-then $\partial X_{i}^{s} / \partial U_{i}$ may be negative. In fact, 
if $c_{i}$ is big enough relative to the other parameters, we could find

$$
\frac{\partial}{\partial U_{i}}\left(X_{i}^{s}+Y_{i}^{s}\right)<0
$$

as the rate of recruitment into a very active group increases, the equilibrium size of the group may actually decrease. For $i \neq h$, one can sec directly in (41) how the size of $c_{h}$ relative to the other $c_{j}$ 's determines the impact of an increase in $U_{h}$ on the size of $X_{j}^{s}$. In particular, increases in the recruitment rate of a very active or very infective subgroup have a negative effect on the equilibrium populations of other groups.

For proportional mixing, the effect of an increase in $U_{h}$ on the equilibrium infected fraction of any group $i$ is the same for all groups $i$ and is determined by the sign of $\Sigma_{j}\left(c_{h}-c_{j}\right) U_{j}$. If $c_{h}=\max c_{j}$, then all infected fractions increase as $U_{h}$ increases; if $c_{h}=\min c_{j}$, then all infected fractions decrease as $U_{h}$ increases.

The effect of $U_{i}$ on the threshold condition also depends on the sign of $\Sigma_{j}\left(c_{i}-c_{j}\right) U_{j}$; for groups with a relatively high activity rate, an increase in the recruitment rate will make the endemicity threshold easier to attain, with the opposite for groups with low contact rates.

For preferred mixing, the results are even more complex than they are for proportional mixing. In Appendix D, we show that for a group $i$ with a low activity level or a lower than average infected fraction, increasing the recruitment rate increases the equilibrium size of the noninfected population $X_{i}^{s}$; however, it has an indeterminate effect on the populations of the other groups.

The Proportion of Within-Group Activity $\rho_{i}$. Any discussion of the effect of a change in the proportion $\rho$ of activity that is reserved for within-group interactions applies only to preferred mixing. For groups with a lower than average activity level, as quantified by (40), increasing within-group mixing decreases the infected fraction for that group since it cuts down on interactions with the more highly infected groups. See, for example, Figure 4 (Section 11). The effect on the infected fractions of other groups is indeterminate. On the other hand, if group $i$ has a higher than average equilibrium infected fraction and if $c_{i}\left(1-\rho_{i}\right) Y_{i}^{s}$ and $c_{j}\left(1-\rho_{j}\right) X_{j}^{s}$ are much larger than the other $c_{h}\left(1-\rho_{h}\right) X_{h}$ and $c_{h}\left(1-\rho_{h}\right) Y_{h}$, then decreasing group $i$ 's outsidethe-group activity decreases group $j$ 's infected fraction.

The effect of a change in $\rho_{i}$ on the threshold of endemicity is rather complex because of the complex form of this threshold. If, for example, $\rho_{i}$ is small, then increasing $\rho_{i}$ makes an endemic equilibrium easier to reach.

The Mean of the Incubation Times, $m / k$. Recall that $m$ represents the number of stages of the disease and $k$ is the fractional transfer rate from one stage to the next. For $\mu=0$, these parameters appear only as $m / k$ in Table 1. Recall from Section 4 that $m / k$ is the mean of the density of incubation 
times for a chain of $m$ compartments with uniform fractional transfer coefficient $k$. One computes directly from the entries in Table 1 that for all three types of mixing, as $m / k$ rises,

Each $X_{i}^{s}$ falls.

Each $Y_{i}^{s}$ rises.

Each infected fraction $Y_{i}^{s} /\left(X_{i}^{s}+Y_{i}^{s}\right)$ rises.

The endemicity threshold rises, so it is more likely for the endemic equilibrium to arise.

Note that $m / k$ rises as either the number $m$ of stages rises or as the fraction $k$ that moves per unit time from one stage to the next falls.

\section{THE REPRODUCTIVE RATE, THRESHOLDS, AND ENDEMIC STATES}

In this section we relate the classical ideas of reproductive rate and the thresholds derived therefrom with the results we have obtained.

\section{THE REPRODUCTIVE RATE}

A key idea in the mathematical theory of epidemics and of endemicity is the number of secondary cases that a case can generate during its infectious period. First used by MacDonald [81], the idea is now a standard basic concept in the epidemiology literature [82, 83]; but we note here that it was introduced from consideration of homogeneous populations. Let $D$ be the mean infectious period, let $c$ be the number of contacts per unit time, and let $\beta$ be the probability of transmission in a contact. Then one infective has $\lambda=\beta c$ contacts per unit time that can lead to transmission (effective contacts). If $S$ is the fraction of susceptibles in the population, only $\beta c S$ effective contacts per unit time occur with susceptibles, and then $\beta c S D$ is the number of effective contacts with susceptibles during the infectious period for one infective. This number is therefore the number of cases generated per infective and is called the reproductive rate $R$. Hethcote [46, 53] calls $R$ the infective replacement number. If one infected is introduced into a large susceptible population, $S=1$, and $R$ is then called the initial reproductive rate, $R_{0}=\beta c D$.

From $R$ and $R_{0}$ one obtains the classical thresholds. For an epidemic to take off after the introduction of one infected, $R_{0}$ must be greater than 1 , giving the condition $\beta c D>1$. If an epidemic develops, the fraction of susceptibles decreases and the epidemic wanes; how it wanes depends on whether infecteds recover with or without immunity or die. If more susceptibles appear, by recruitment of new ones or by recovery without immunity, an endemic state can appear when each infected is replaced by exactly one infective, i.e., $\beta c D S=1$. Since $0<S<1$ in an endemic state, this also gives 
the condition

$$
\beta c D>1 .
$$

This is all very sensible for a homogeneous population with random mixing. For a heterogencous population with random, i.e., proportional, mixing, May and Anderson obtain Equation (42) but with $c$ replaced by a quadratic weighted average [67, 68]. [In addition, see Equations (C24) and (C25) in Appendix C.] Can this idea be generalized to heterogeneous populations with nonrandom mixing?

\section{GENERALIZATION TO HETEROGENEOUS POPULATIONS}

Consider a heterogeneous population made up of $n$ subgroups. The natural extension of the idea of a reproductive rate is to consider a reproductive or replacement rate matrix $\mathbf{R}$; the element $R_{i j}$ is the number of cases generated in group $i$ by one case in $j$. Thus, $R_{i j}$ must be the product of

(1) The number of contacts per unit time of one infected in $j$ with people in $i, c_{j} \rho_{j i}$,

(2) The transmission fraction for transmission from $j$ to $i, \beta_{j i}$,

(3) The fraction susceptible in $i, S_{i}$, and

(4) The mean duration of infectiousness for a person in $j, D_{j}$ :

$$
R_{i j}=c_{j} \rho_{j i} S_{i} \beta_{j i} D_{j} .
$$

For the purposes of this paper, we consider populations for which the transmission fraction and mean duration are the same for all groups, $\beta_{j i}=\beta$ and $D_{j}=D$. Then, if $\mathbf{S}$ is the diagonal matrix of susceptible fractions and $\mathbf{C}$ is the diagonal matrix of contacts per unit time, we can write

$$
\mathbf{R}=\beta D \mathbf{S P}^{T} \mathbf{C} .
$$

Here, $\mathbf{P}$ is the mixing matrix.

Now let $\mathbf{Y}^{s}$ be the column vector of infecteds at the steady state. The conditions for detailed endemicity must be

$$
\mathbf{Y}^{s}=\mathbf{R}^{s} \mathbf{Y}^{s}=\beta D \mathbf{S}^{s} \mathbf{P}^{s T} \mathbf{C Y}^{s},
$$

in which $\mathbf{P}^{s}$ is the value of $\mathbf{P}$ at the steady state. By detailed or stable endemicity, we mean that the vector $\mathbf{Y}^{s}$ is reproduced by components. We can also define an overall endemicity by requiring only that the total number of infectives be constant but allowing the distribution over the subgroups to vary with time. We do not explore the latter interesting possibility here. 
We can also look for epidemic thresholds by assuming the introduction of one or a few infecteds into some subgroups and asking whether the first few terms of the series

$$
\mathbf{Y}_{k+1}=\mathbf{R}_{k} \mathbf{Y}_{k}
$$

form an increasing series for the sums of the components of $\mathbf{Y}_{k}$. Then, for the introduction of one infected, there are $n$ cases to explore; there are interesting facets to this that we cannot explore in the context of this paper.

A GENERAL THRESHOLD FOR OVERALL ENDEMICITY FOR

HETEROGENEOUS POPULATIONS

We now demonstrate a general threshold for overall endemicity for heterogeneous populations of all kinds.

Form the column sums of Equation (44):

$$
\sum_{i} Y_{i}^{s}=\beta D \sum_{j} \sum_{i} S_{i}^{s} c_{j} \rho_{j i}^{s} Y_{j}^{s}
$$

Equation (46) can be written in the form

$$
\sum_{i} Y_{i}^{s}=\beta D\left[\frac{\sum_{j}\left(\sum_{i} S_{i}^{s} \rho_{j i}^{s}\right) c_{j} Y_{j}^{s}}{\sum_{i} c_{i} Y_{j}^{s}}\right] \sum_{j} c_{j} Y_{j}^{s} .
$$

Now note that $\sum c_{j} Y_{j}^{s}$ is the total number of contacts of infecteds and the term in square brackets is the fraction of those contacts that are with susceptibles, which we call $S_{X Y}: 0 \leqslant S_{X Y} \leqslant 1$. Dividing Equation (47) by $\sum Y_{i}^{s}$ gives us the condition for endemicity:

$$
1=\beta D S_{X Y} \bar{c}_{Y}
$$

Here, $\bar{c}_{Y}$ is the infecteds' weighted average of the contact rates, Equation (24). Since we assumed $\Sigma Y_{i}^{s}>0$, we conclude that $0<S_{X Y}<1$, and we obtain the threshold condition

$$
\beta D \bar{c}_{Y}>1
$$

as the general analog of Equation (42).

Equation (49) is obviously a general threshold statement for all populations and provides considerable insight. However, to use it for any particular problem, one has to know the $Y_{i}^{s}$, and that involves solving for the endemic steady states for that model. Note that $\bar{c}_{Y}$ does not depend explicitly on the structure of the mixing matrix; it depends on it implicitly through its effect on the $Y_{i}^{s}$. 
RELATION TO RESULTS OBTAINED ON OUR MODEL

Now we compare the results obtained on our models with this general threshold condition (49) for heterogeneous populations.

Restricted Mixing. For restricted mixing, $\rho_{i i}=1$ and $\rho_{i j}=0$ for $i \neq j$. We see immediately that the thresholds obtained from the model are the same as those given by the requirement of detailed endemicity, for $\mu=0$ and for $\mu>0$ :

$$
\beta D S_{i}^{s} c_{i}=1
$$

Multiplying (50) by $Y_{i}^{s}$ and summing, one obtains the condition for overall endemicity:

$$
\sum Y_{i}^{s}=\beta D \sum S_{i}^{s} c_{i} Y_{i}^{s}
$$

which is the same as Equation (48) and gives the endemicity condition $\beta D \bar{c}_{Y}>1$.

Proportional Mixing. For $\mu=0$, we obtained the threshold condition $\beta D \bar{c}_{U}>1$, Equation (32), which is the same as (49) since $\bar{c}_{U}=\bar{c}_{Y}$ for $\mu=0$. In Appendix $C$, we obtain two thresholds for $\mu>0$; one is the general relation (49), and the other is (52) or (C25):

$$
\beta D c^{*}>1 \text {, }
$$

where $c^{*}=\bar{c}_{U}+\sigma^{2} / \bar{c}_{U}=\sum c_{j}^{2} U_{j} / \sum c_{j} U_{j}$. What is the relation between these two? Using relation (C20) for $Y_{i}^{s}$ for proportional mixing, one obtains

$$
\bar{c}_{Y}=\frac{\sum_{j} c_{j}^{2} U_{j} /\left(\mu+c_{j} \bar{\beta} K_{Y}^{s}\right)}{\sum c_{j} U_{j} /\left(\mu+c_{j} \bar{\beta} K_{Y}^{s}\right)}
$$

Thus, $c^{*}=\bar{c}_{Y}$ calculated for $K_{Y}^{s}=0$, which is just the condition used in deriving this threshold in Appendix C. Note that Equation (53) differs from the quadratic average used by Anderson et al. [68].

Preferred Mixing. If one substitutes the steady-state values of $Y_{i}^{s}$ obtained in Section 7 and Appendix C into Equation (44), one obtains the same equations for the endemic state as are given by the differential equations, so the solutions must be the same.

For $\mu=0$, we obtained (B7) in Appendix B, which is the same as our overall threshold for endemicity. For $\mu>0$, the relation between Equation (C40) of Appendix C and our general threshold condition is not obvious, and we have not pursued this. 
TABLE 3

\begin{tabular}{cccc}
\multicolumn{4}{c}{ Contact Activity Groups } \\
\hline Group & $\begin{array}{c}\text { Number of } \\
\text { partners/year }\end{array}$ & $\begin{array}{c}\text { Pooled groups } \\
\text { from Fig. 4a of May } \\
\text { and Anderson [67] }\end{array}$ & Fraction \\
\hline 1 & 12 & 1 & 0.24 \\
2 & 24 & 2 & 0.24 \\
3 & 48 & $3-5$ & 0.32 \\
4 & 96 & $6-10$ & 0.14 \\
5 & 192 & $>10$ & 0.06 \\
\hline
\end{tabular}

\section{PARAMETER VALUES AND INITIAL CONDITIONS}

For the numerical studies of the dynamics and the steady states, we had to choose contact activity groups, recruitment rates for these groups, initial sizes of the groups, and values for the transmission fractions.

\section{CONTACT ACTIVITY GROUPS}

We use the data reported in Figure 4a in May and Anderson [67] on the distribution of the number of partners per month in a group of homosexual/bisexual males in London. The data were pooled into five activity groups after dropping the group with zero contacts per month and are reported in Table 3.

\section{COMPETING DEATH RATES}

The competing death rate $\mu$ is really the total rate at which members leave the groups from all causes other than the development of AIDS. From the U.S. vital statistics, the actual deaths from all causes in white males in the 20-50-year-old group would give a $\mu$ on the order of 0.003 per year. If we assume that the average length of time a homosexual remains sexually active is 40 years, we obtain $\mu=0.025 \mathrm{yr}^{-1}$. We have run simulations for $\mu=0.03 \mathrm{yr}^{1}$ and $\mu=0.012 \mathrm{yr}^{1}$.

\section{RECRUITMENT RATES AND INITIAL CONDITIONS}

For the steady-state studies we chose a hypothetical population that recruits a total of 100 per month ( 1200 per year), divided into activity groups according to the fractions given in Table 3; for example, 24 per month in group 1. For $\mu=0.03 \mathrm{yr}^{-1}$ and in the absence of HIV, that would give a steady-state population of 40,000 individuals: 9600 in each of groups 1 and 2,12800 in group 3, 5600 in group 4 , and 2400 in group 5 . With $\mu=$ 
$0.012 \mathrm{yr}^{-1}$ and in the absence of HIV, the steady-state population consists of 100,000 individuals: 24,000 in each of groups 1 and 2, 32000 in group 3, 14000 in group 4 , and 6000 in group 5 . For given $\mu$, we used the stationarystate population in the absence of HIV for the initial values for the simulations of the dynamics after introduction of HIV.

\section{INCUBATION TIMES}

As has been pointed out, Weibull and gamma distributions have been used for the distribution of incubation times $[17,18,20]$. The gamma distribution has a skewed shape similar to the Weibull distribution and is a natural choice for us because a chain of compartments gives a lag with a gamma distribution. For the numerical studies we have chosen two mean values: 5 and $10 \mathrm{yr}$. Most of the simulations were done with $m=6$ and $k=1.2 \mathrm{yr}^{-1}$; these give a mean of $5 \mathrm{yr}$, a mode of $4.17 \mathrm{yr}$ and a standard deviation of $2.04 \mathrm{yr}$, figures close to one of the estimates of Lui et al. [17]. For comparison we have also run some simulations with $m=6$ and $k=$ $0.6 \mathrm{yr}^{-1}$; these give a mean of $10 \mathrm{yr}$, a mode of $8.33 \mathrm{yr}$, and a standard deviation of $4.08 \mathrm{yr}$.

\section{THE TRANSMISSION FRACTION}

Data from which one can estimate transmission probabilities are limited, and the corresponding estimates are subject to uncertainties. For one, from what we know of sexually transmitted diseases and the mucosal protective barrier, we expect that any breaks in continuity of mucous membranes in a partner in a sex act should increase the probability of transmission to that partner. Thus, for any given mode of transmission, the population may be heterogeneous in transmission probability. For a discussion of many of the problems of interpretation of the data, see May and Anderson [84]. Peterman et al. [42] reported data on heterosexual transmission from a spouse infected by a blood transfusion; the number of contacts from infection of spouse to detection of HIV in a person varied markedly. If one treats the data as independent runs of variable length, of independent trials, the maximum likelihood estimate for transmission probability per contact is 0.0013 for male-to-female and 0.0005 for female-to-male transmission. On a per-partnership basis, the corresponding figures are 0.189 and 0.08 . Grant et al. [40] provide estimate of per-partnership infectivity for anal receptive intercourse in homosexuals of 0.102 , with a $95 \%$ confidence interval of 0.043-0.16; data on numbers of contacts were not obtained.

Given the uncertainties, we have run simulations with a range of values. For the steady state, we have examined results for a number of values of the mean transmission fraction in the range $0.005-0.1$. For the studies of dynamics, we compared a series of cases that had the same mean transmis- 
TABLE 4

\begin{tabular}{ccccc}
\multicolumn{4}{c}{ Distributions of Transmission } & \multicolumn{4}{c}{ Fraction Examined in Studies of Dynamics } \\
\hline & Case & Case & Case & Case \\
& I & II & III & IV \\
\hline$\beta_{1}$ & 1.0 & 2.2 & 2.5 & 0.25 \\
$\beta_{2}$ & 1.0 & 0.2 & 2.5 & 0.25 \\
$\beta_{3}$ & 1.0 & 0.1 & 0.25 & 0.25 \\
$\beta_{4}$ & 1.0 & 0.2 & 0.25 & 0.25 \\
$\beta_{5}$ & 1.0 & 1.0 & 0.25 & 2.5 \\
$\beta_{6}$ & 1.0 & 2.3 & 0.25 & 2.5 \\
\hline
\end{tabular}

${ }^{a}$ Table entries are multiplies of $\vec{\beta}$.

sion fraction, $\bar{\beta}=0.005$ and 0.01 , but different distributions over the infectious period (Table 4). Case II with high transmission fraction early and late in the infectious period is intended to simulate the reported increase in free virus that occurs early after contracting HIV and again late just before the appearance of AIDS.

In this study, we have not tried to simulate details of survival once AIDS is diagnosed. For that, $Z_{i}$ could be replaced by a sequence of compartments with mean transit time set equal to the mean survival time.

\section{RESULTS OF STUDIES OF THE STEADY STATE}

Figure 4 shows the steady-state infected fraction for $\beta=0.01$ and $\mu=$ $0.03 \mathrm{yr}^{-1}$ for $k=1.2 \mathrm{yr}^{-1}$ as a function of the fraction of contacts reserved to each group if the reserved fraction $\rho_{i}$ is the same for all $i$. For comparison, we also show the results of two cases in which the reserved fraction increases from 0.05 to 0.98 for groups $c_{1}=12 \mathrm{yr}^{-1}$ to $c_{5}=192 \mathrm{yr}^{-1}$ and the inverse of that, i.e., one in which $\rho_{1}=0.98$ and $\rho_{5}=0.05$. Those results are connected by dashed lines. The results for $\mu=0$ differ slightly from those for $\mu=0.012$ or 0.03 .

The difference between the effects of the mixing patterns on the steadystate fraction infected in high- and low-activity groups is striking. For the high-activity groups, the mixing pattern has only small effects on the fraction infected. However, for the low-activity groups, the fraction infected is markedly increased by mixing with high-activity groups, just as predicted by the theory in Section 8 and in Theorem D.4. Even though the contact rates remain constant, the less mixing with high-activity groups, the lower the prevalence of HIV infection in the low-activity groups. 


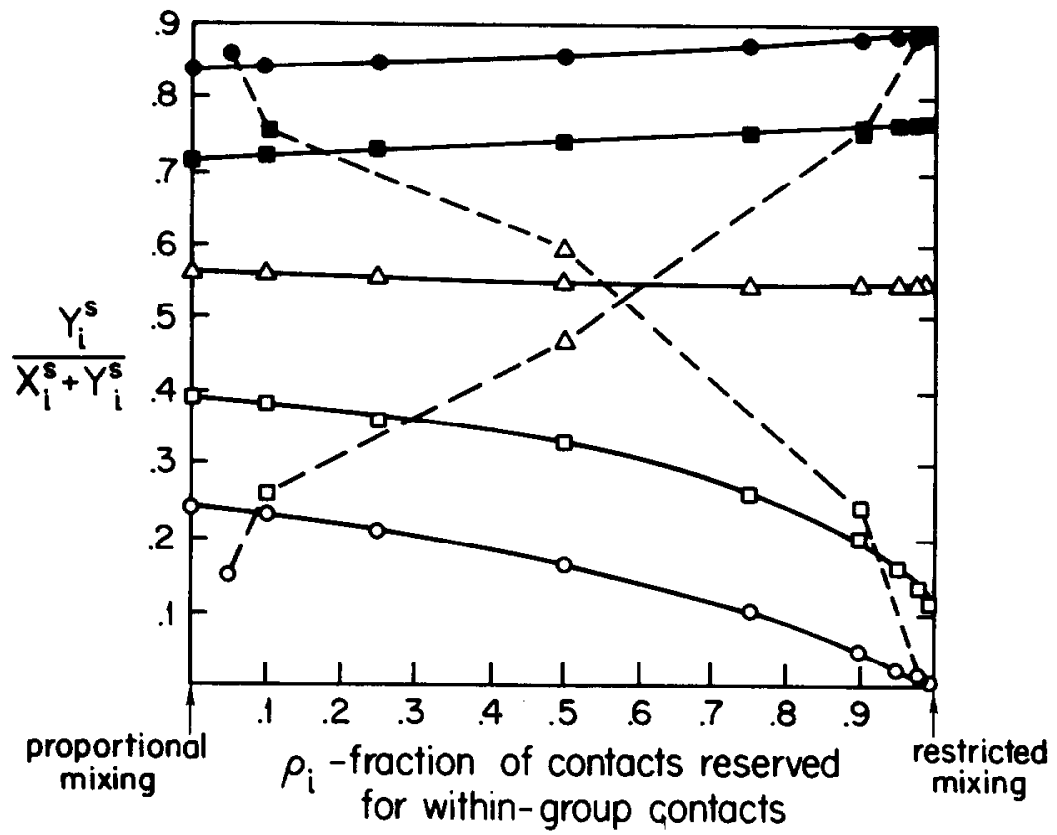

FIG. 4. The steady-state infected fraction for $\beta=0.01, \mu=0.03 \mathrm{yr}^{-1}, k=1.2 \mathrm{yr}^{-1}$. Solid lines show how the fraction infected varies from proportional to restricted mixing if all groups have the same fraction of their contacts reserved for within-group contacts. The dashed lines connect the different groups in simulations in which the groups had different $\rho_{i}$. Groups: $O, c_{1}=12 ; \square, c_{2}=24 ; \Delta, c_{3}=48 ; \boldsymbol{\square}, c_{4}=96 ; \bullet, c_{5}=192$ per year.

The time courses to the steady states emphasize even more strongly the differences in the effects of mixing on low- and high-activity groups, as can be seen in the following results of the studies on dynamics.

\section{STUDIES ON DYNAMICS}

For the first studies on dynamics, the compartmental modeling software CONSAM29 $[85,86]$ was used on the ATT Unix PC. This software package can be used to integrate the system equations and to estimate parameters. However, the software we have is limited to systems of 25 compartments or less, so we ran only groups 1,3 , and 5 in the dynamics. The simulation studies with all five groups were run using STELLA [87] on a Macintosh II. We have run simulations for $\beta=0.005$ and 0.01 for the four cases shown in Table 4 , for $\mu=0.012$ and $0.03 \mathrm{yr}^{-1}$, and for various $\rho_{i}$. In addition, runs have been made with $\beta=0.02$ and also for a model in which the infectious 


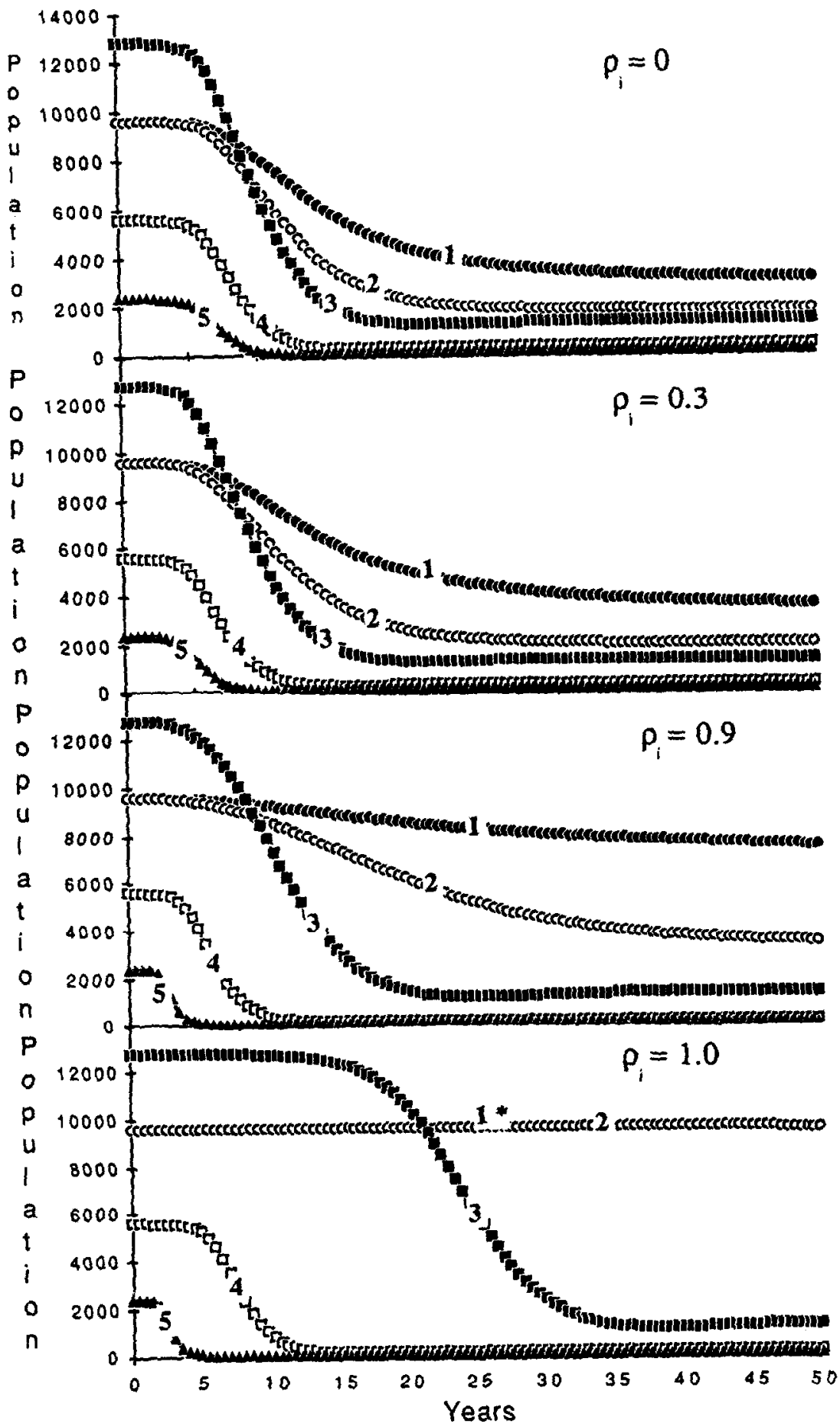

F1G. S. The change in the size of the susceptible population after introduction of one infected in stage 1 of the infection, into each group, for proportional, preferred, and restricted mixing. The fraction of a group's contacts reserved to the group, $p_{i}$, is the same for all groups in a min. Case II for $\beta=0.01, k=1.2 \mathrm{yr}^{-1}, \mu=0.03 \mathrm{yr}^{-1}$. Group 1 is below threshold for restricted mixing $\left(\rho_{i}=1\right)$ and remains constant. Group 2 is just above threshold for restricted mixing and actually decreases very slowly. 


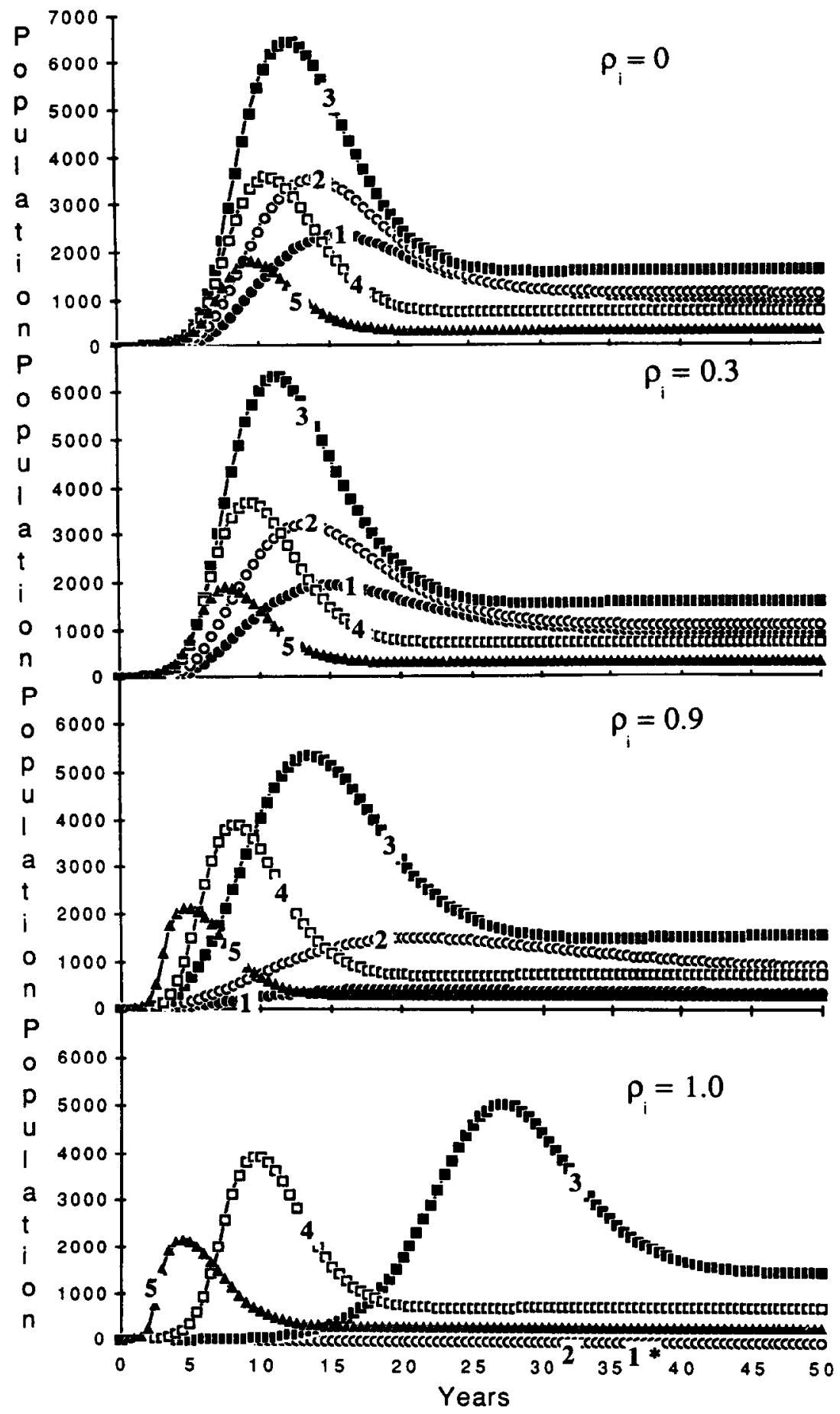

Fig. 6. Number of infecteds for the same runs as in Figure 5. 


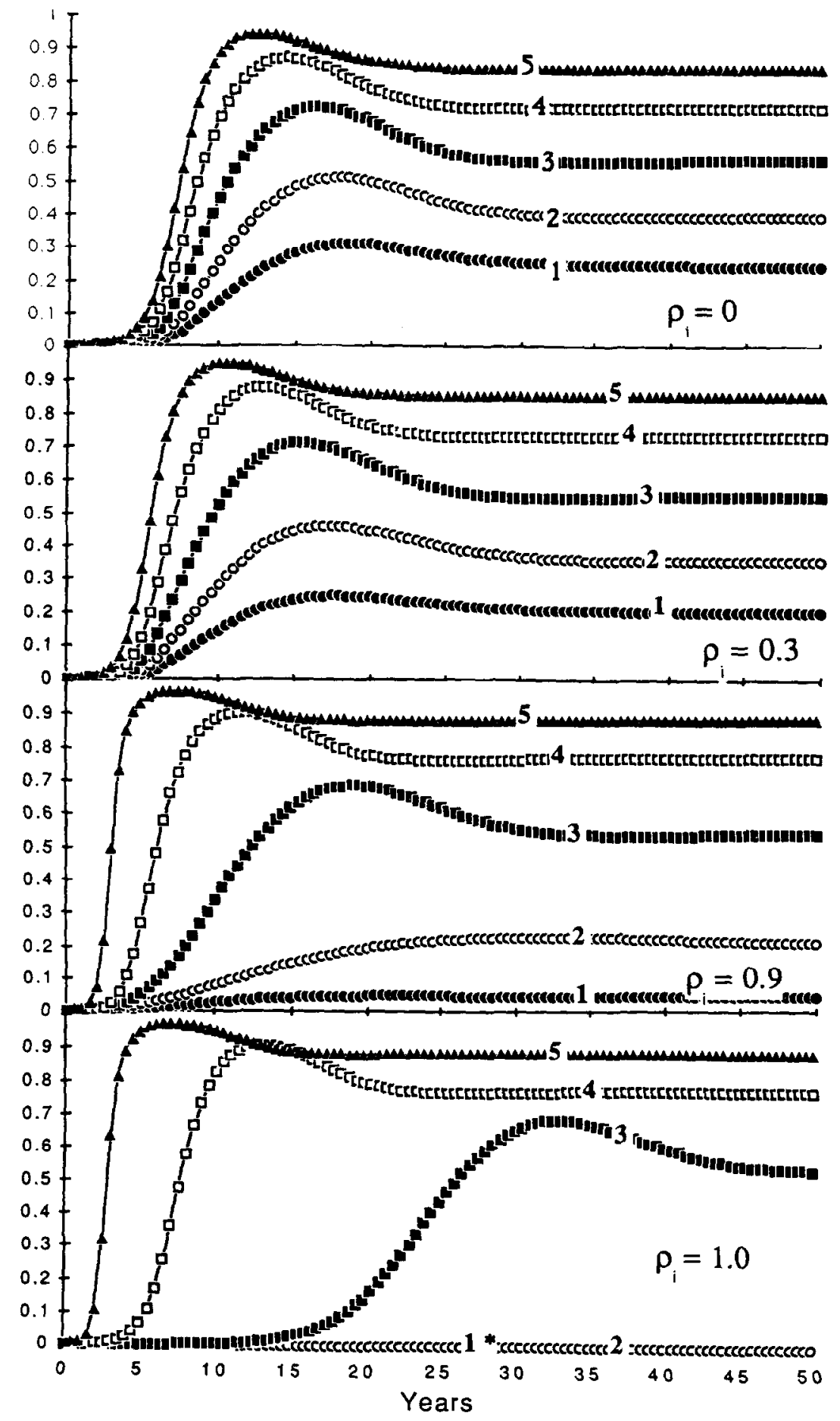

FIG. 7. Fraction infected for the same runs as in Figure 5. 
period was modeled with four compartments with different $k$ values $\left(k_{1}=\right.$ $\left.0.5, k_{2}=3, k_{3}=1, k_{4}=1\right)$ and $\beta_{i j 1}=0.03, \beta_{i j 2}=0, \beta_{i j 3}=\beta_{i j 4}=0.03$ for various values of $\rho_{i}$.

In the runs, one infected person in stage 1 of infection was introduced into each group that was at stationary state for the given $\mu$ at $t=0$, and the dynamics was followed out to or close to steady state. The output plotted was the number of susceptibles $X_{i}$, the number of infecteds $Y_{i}$, and the percent infected $Y_{i} /\left(X_{i}+Y_{i}\right)$, by groups, plus the infected ratio, the sum of susceptibles and infecteds, and the incidence of AIDS for the total population.

Although the quantitative results vary some, the different simulations gave qualitatively similar results. We present one set of results for $\bar{\beta}=0.01$ for a mean incubation period of $5 \mathrm{yr}\left(k=1.2 \mathrm{yr}^{-1}\right)$ in detail for case II (Table 4) and show the effects of a change in $\bar{\beta}$ and $k$ and then compare the results with some runs on cases III and IV. Figures $5-7$ give the results for all groups for case II for $\bar{\beta}=0.01, k=1.2 \mathrm{yr}^{-1}, \mu=0.03 \mathrm{yr}^{-1}$ for proportional mixing $\left(\rho_{i}=0\right)$, preferred mixing $\left(\rho_{i}=0.3\right.$ and 0.7$)$, and restricted mixing $\left(\rho_{i}=1\right)$. In these runs the fraction of contacts reserved to within-group contacts $\left(\rho_{i}\right)$ was the same for all groups. For those values of the parameters, group 1 is below threshold for restricted mixing but not otherwise. Note that the fraction infected does not differ much eventually for the high-activity groups, no matter what the pattern of mixing, a result already shown in Figure 4. However, the fraction infected rises sooner for high-activity groups as one approaches restricted mixing because contacts with the low-activity groups slows down spread in the high-activity groups. However, for the low-activity groups, contact with high-activity groups increases spread.

\section{Infected Fraction -- $Y /(X+Y)$}

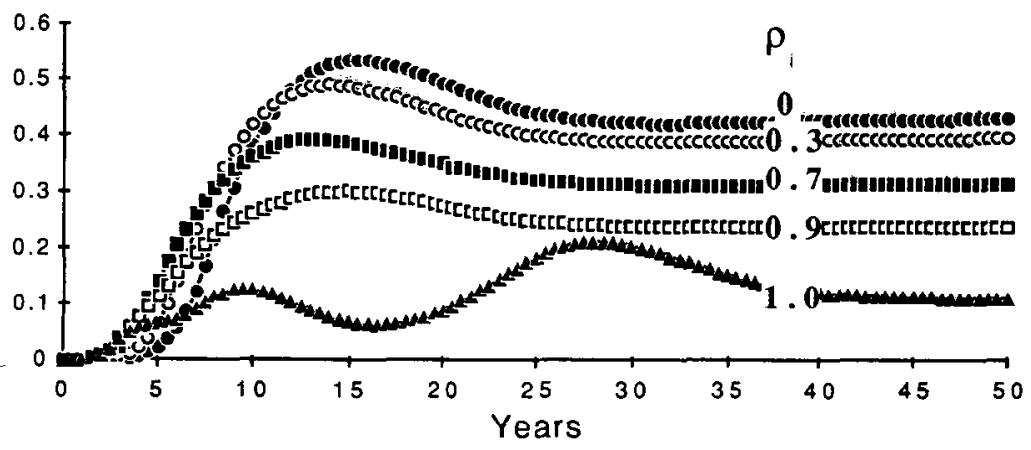

FIG. 8. The fraction infected for the total population, $\Sigma Y_{i} /\left(\sum X_{i}+\sum Y_{i}\right)$, for the simulation shown in Figures 5.7 for $\rho_{i}-0,0.3,0.7,0.9$, and 1.0. 


\section{Total Population}

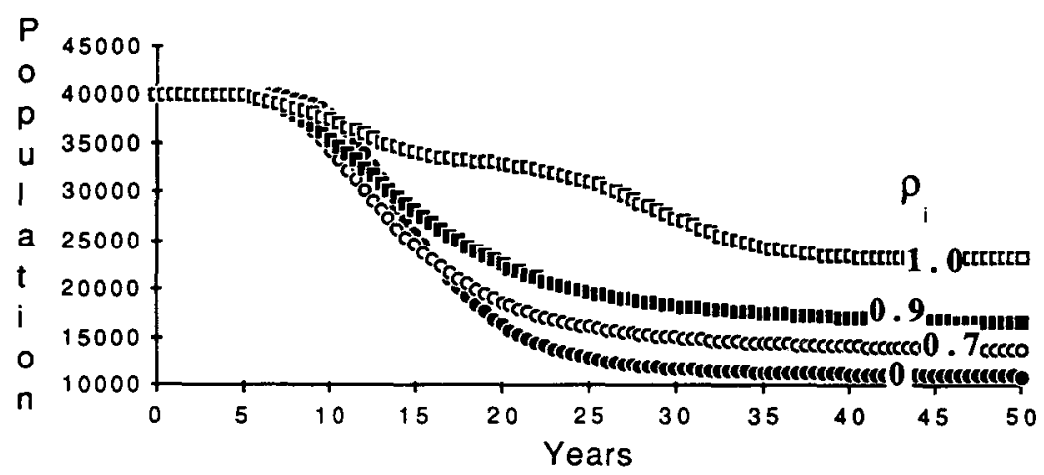

Fig. 9. The size of the population, susceptibles plus infecteds, for the runs shown in Figures 5-8.

The curve for the infected fraction in the population as a whole (Figure 8) reflects the effects of mixing. The epidemic develops more slowly in full proportional mixing because, as seen in the curves on individual groups, the contact between low- and high-activity groups slows down the spread in high-activity groups. As mixing is decreased, the epidemic in the population takes off more rapidly because it spreads more quickly in the high-activity groups although more slowly in the lower-activity groups. If mixing between groups is very limited, the curve becomes biphasic, reflecting the marked differences in spread in the almost isolated subgroups. Figure 9 shows the effect of AIDS on the size of the population and the effect of the mixing thereon; the more mixing between groups the more AIDS cases in the lower-activity groups, as Theorem D.4 asserts. The high-activity groups are badly hit for all patterns of mixing. Figure 10 shows the total incidence of AIDS for the population and shows how strongly the pattern of the epidemic depends on the mixing pattern in the population.

Figure 11 is included to show what happens in group 1, which is below threshold for $\rho_{i}=1$, as $\rho_{i}$ approaches 1 . The steady-state fraction infected falls steadily, and as it does, the rate of take-off of the curves decreases.

Figures 12 and 13 show the effects of decreasing $\bar{\beta}$ to 0.005 , all other parameters being the same as for the runs in Figures 5-10. The steady-state levels of the fraction infected decrease, as Theorem D.3 predicts; but in addition the time course of spread is slowed. For restricted mixing, groups 1 and 2 are now below threshold. As is shown in Figure 13, the epidemic of AIDS is much smaller and develops more slowly. 


\section{Incidence of AIDS}

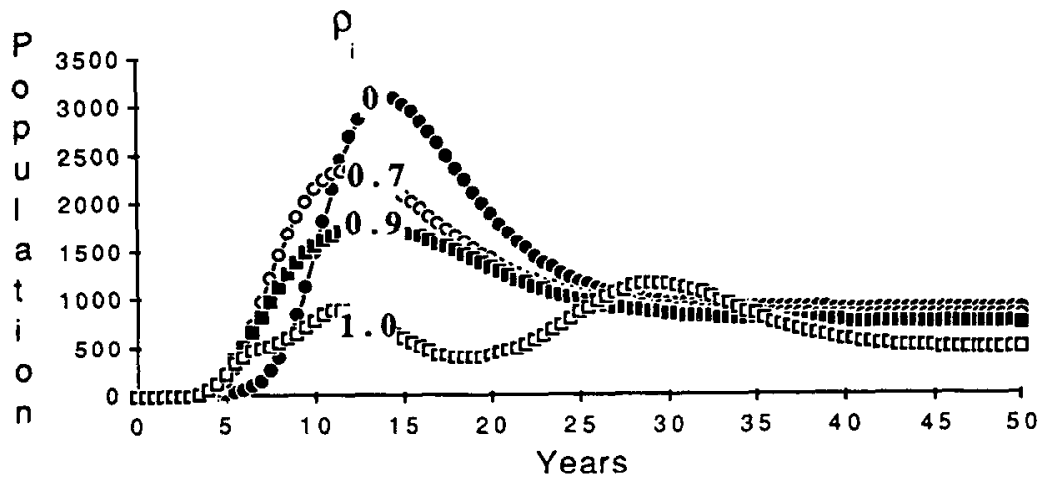

Fig. 10. The incidence of AIDS (number of cases per year) for the runs shown in Figures 5-9.

\section{Infected Fraction $\quad--Y_{1} /\left(X_{1}+Y_{1}\right)$}

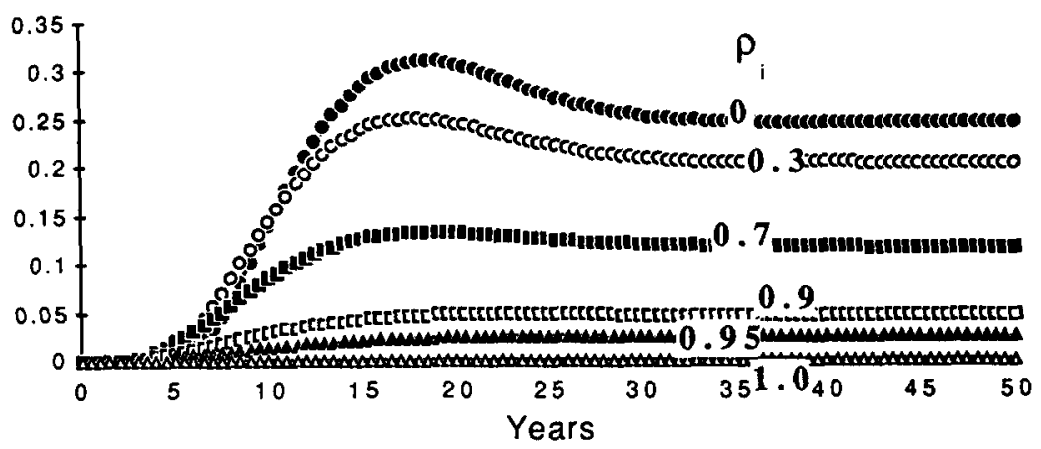

FIG. 11. Ratio of infecteds for group $1\left(c_{1}=12 \mathrm{yr}^{-1}\right)$ for $\rho_{i}=0,0.3,0.7,0.9,0.95$, and 1.0. Group 1 is below threshold for $\rho_{i}=1.0$.

Figures 14 and 15 are the same as Figures 12 and 13 , respectively $(\bar{\beta}=0.005)$, but for a longer period of infectiousness $\left(k=0.6 \mathrm{yr}^{-1}\right)$. Increasing the infectious period (i.e., decreasing $k$ ) markedly increases the growth of the infected fraction and the steady-state level of the infected fraction, as we computed in Section 8, but has only a small effect on the epidemic of AIDS because the rate of movement from the infected state to AIDS is slower. 


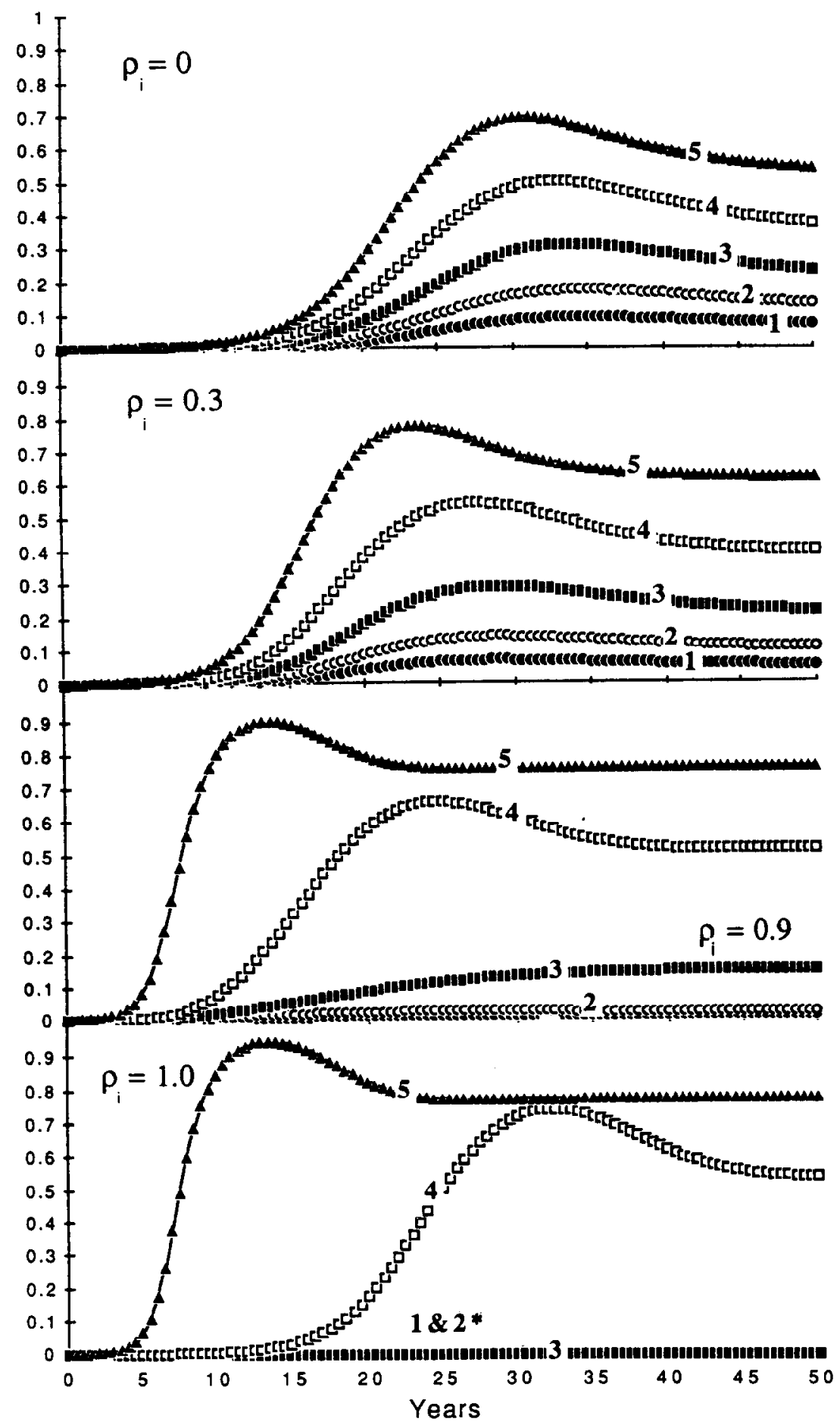

FIG. 12. The fraction infected for groups $1-5$ for the same case as is shown in Figure 7 but for $\beta=0.005$ (one-half of its value for the runs in Figures $5-11$ ). Note that groups 1 and 2 are below threshold for $\rho_{i}=1.0$. 


\section{Total Incidence of AIDS}

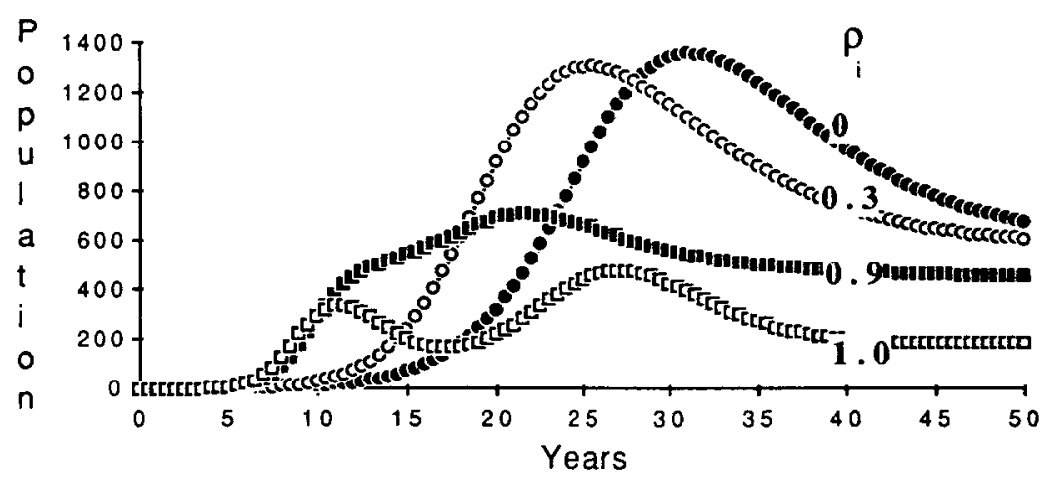

FIG. 13. Incidence of AIDS for the runs shown in Figure 12.

Figures 16 and 17 show the difference between infected fractions for cases III and IV. In case III, infectiousness is high immediately after onset of the infection, whereas in case IV it is high late in the infectious period, but both have the same mean $\bar{\beta}$. Clearly, there is a marked effect on the rate of growth of the infected fraction. We include these to emphasize the importance of obtaining estimates of the pattern of infectiousness over the period of infectiousness.

Figures 18 and 19 show the dynamics when there are marked differences in the reserved fractions. In Figure 18, it was assumed that most mixing in the low-activity groups was reserved for with-in group mixing. In Figure 19, the pattern of reserved fractions was the inverse of that for Figure 18. Again there are only small effects on high-activity groups; it is the low-activity groups that show large increases in infected fraction as mixing with highactivity groups increases.

\section{DISCUSSION AND CONCLUSIONS}

We emphasize that these studies cannot simulate the time course in real populations because we have not included subgroups such as bisexuals, heterosexuals, drug users, and prostitutes. Nor have we included the nature of the contacts between them or the effect of pair formation. This study focuses on the effects of the structure of contacts between different activity groups and the effect of variation in infection fraction over the course of the infectious period in a homosexual population. The results emphasize the dominant influence of the pattern of the contacts between different groups.

The effect of activity level is obvious and expected. Those individuals with the highest level of sexual activity are at greatest risk for the infection, 


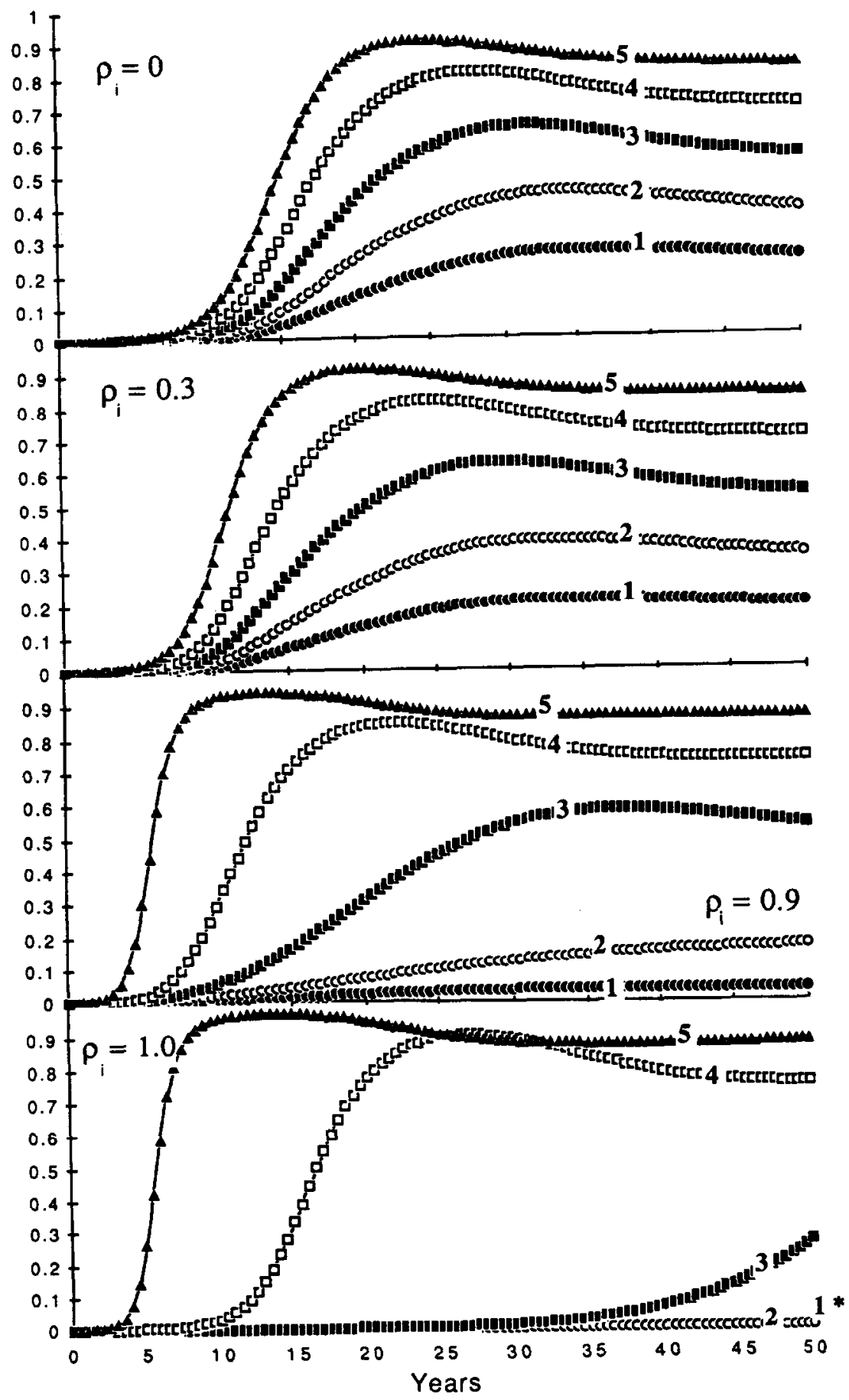

Fig. 14. The fraction infected for the case shown in Figure 12 but for $k=0.06 \mathrm{yr}^{-1}$. With the longer infectious period, there is a large increase in the fraction infected. 


\section{Total Incidence of AIDS}

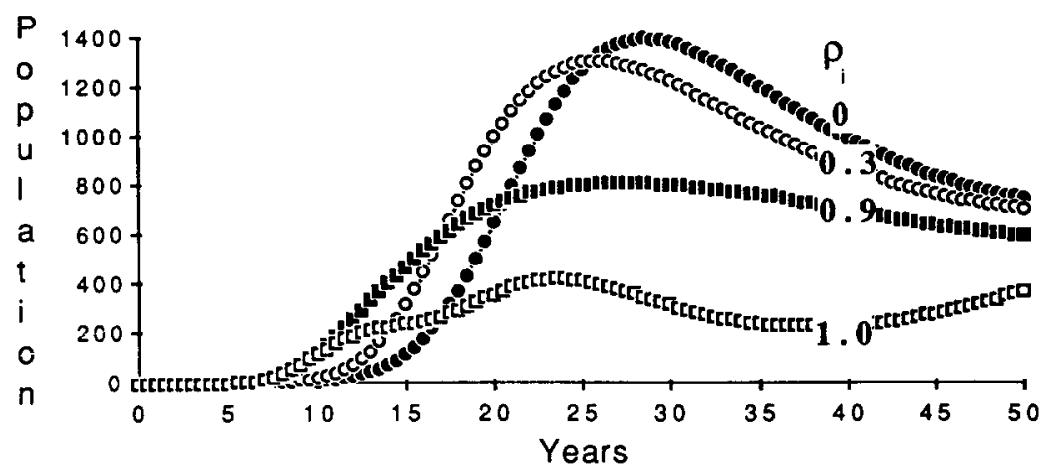
$\mathrm{yr}^{-1}$ ).

FIG. 15. The incidence of AIDS for the runs shown in Figure $14(\beta=0.005, k=0.6$

while those with the lowest levels of sexual activity are at least risk. However, the effect of the structure of the contacts between the groups on the rate of development of the epidemic in the different activity levels is less obvious and is quite striking. The effects of the contact structure are small for the high-activity groups. Increasing contact between high-activity groups and other groups slows the rate of spread of the infection within the high-activity groups a little. However, the rate of spread of the infection within the low-activity group is markedly affected by the degree of contact with higher-activity groups. A slight increase in the amount of interaction between individuals with low levels of sexual activity and individuals with high levels of sexual activity results in a significant increase in the risk for infection of all individuals within the low-activity level. There was both an increase in the rate of spread of the infection and an increase in the overall proportion of the group that ultimately became infected, despite the fact that the total number of contacts and the transmission probabilities remained the same.

Do our results have any implications for the spread of HIV into the heterosexual population? Most of the heterosexual population can be thought of as pairs in a number of low-activity groups, because most of the activity is between members of a pair, and the activity involved in spread is between pairs. Bisexuals, prostitutes, and highly promiscuous individuals form a bridge of contacts to the currently heavily infected groups, contacts that repeatedly introduce HIV into the heterosexual population. Thus the time scale of spread of HIV should be more like that found in our low-activity groups with low mixing with high-activity groups. Consequently, the appar- 


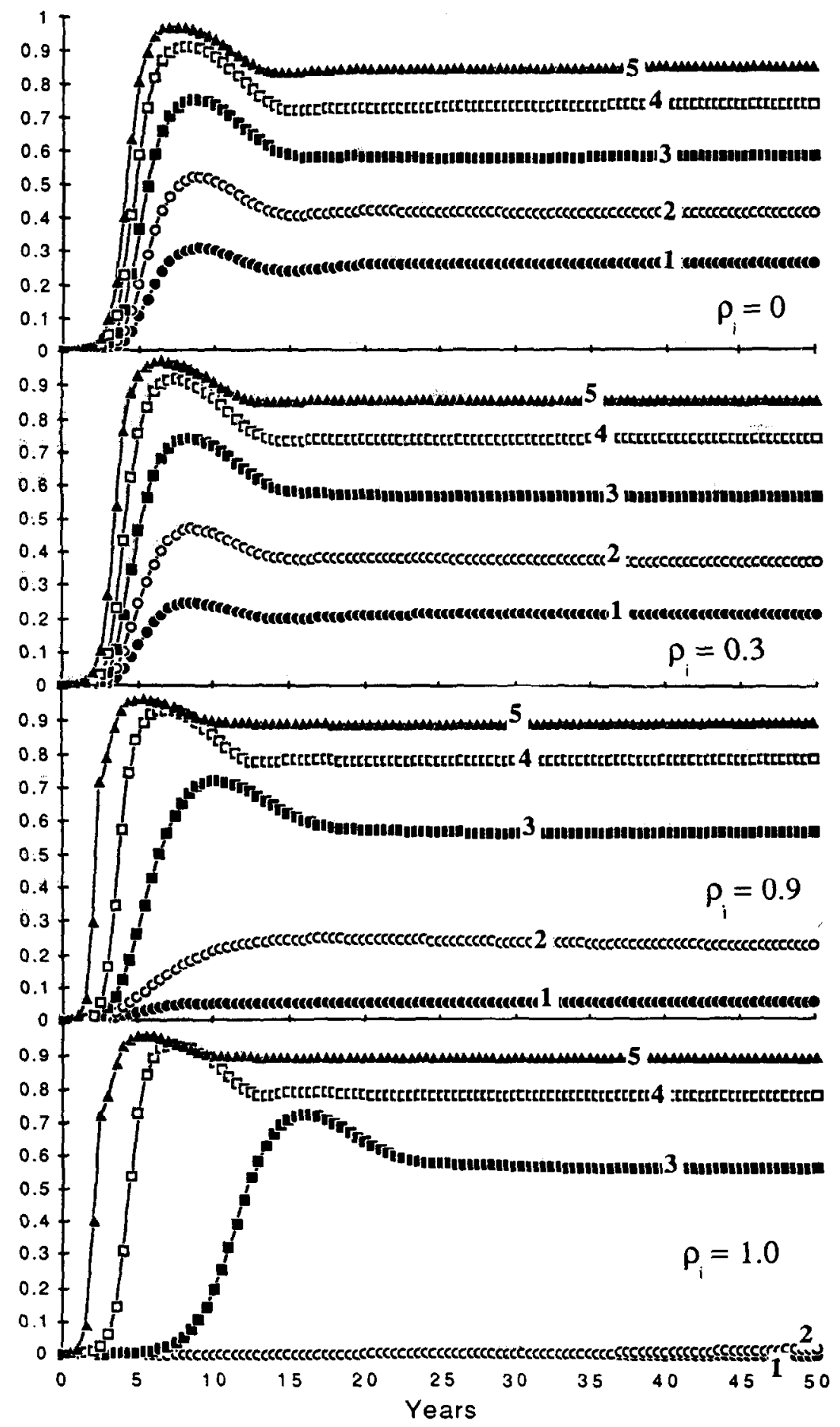

FIG. 16. The infected fraction for the same parameters as in Figures 5-10 but for case III (high infectivity early in the infectious period). 


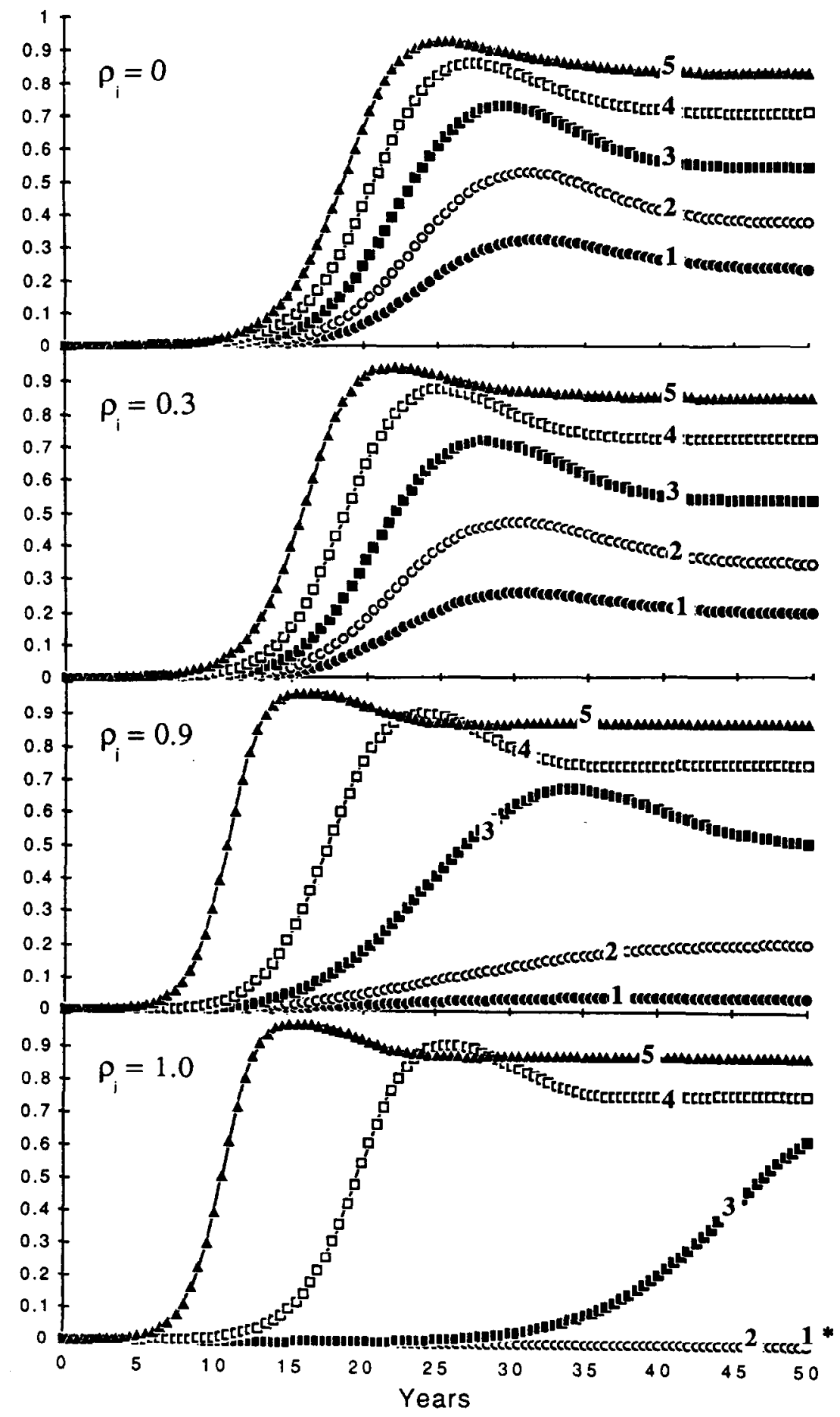

FIG. 17. The infected fraction for the same parameters as in Figures 5-10 but for case IV (high infectivity late in the infectious period). 


\section{Infected Fraction -. Pattern A}

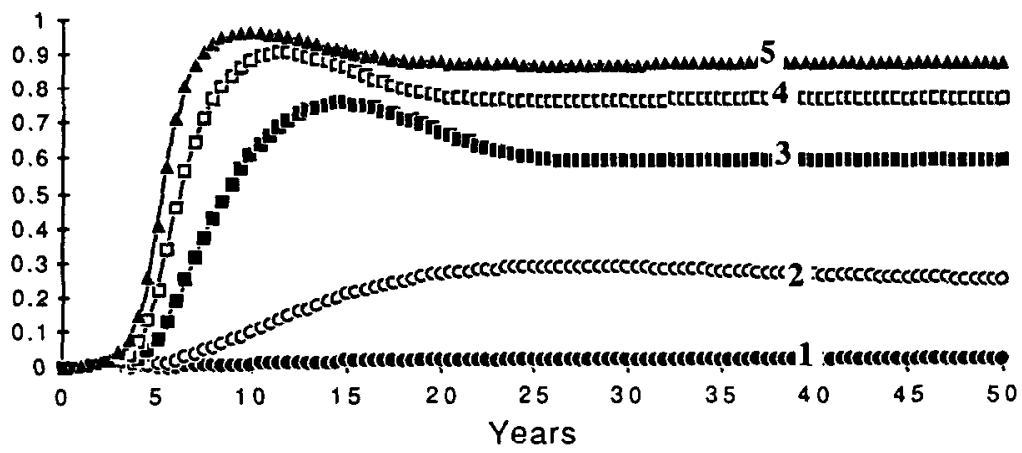

FIG. 18. The time course of the fraction infected for a population with the same parameters as for the population shown in Figure 7, but with different reserved fractions for each group. Pattern A: $\rho_{1}=0.98, \rho_{2}=0.90, \rho_{3}=0.50, \rho_{4}=0.10, \rho_{5}=0.05$. The steady state is shown in Figure 4.

\section{Infected Fraction .. Pattern B}

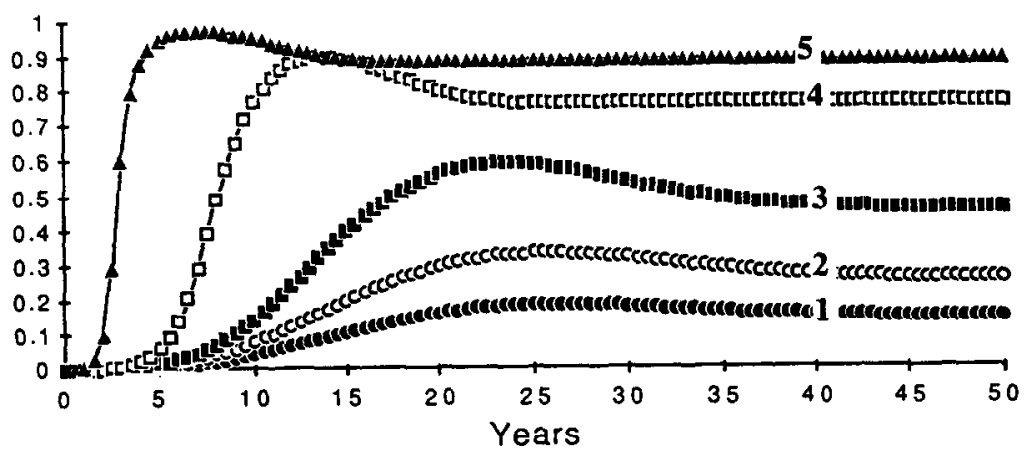

Fig. 19. The same as Figure 18, but with the reserved fractions the reverse of those used for Figure 18. Pattern B: $\rho_{1}=0.05, \rho_{2}=0.10, \rho_{3}=0.50, \rho_{4}=0.90, \rho_{5}=0.98$. The steady state for this population is also shown in Figure 4. 
ently slow rate of spread in the heterosexual population does not mean that the heterosexual population is safe; it could mean that the dynamics is very slow in developing.

The transmission of HIV infection is effectively restricted to sexual contact and blood contamination. This implies to us that intricate patterns of transmission contact deviating dramatically from random mixing patterns are inevitable. The exploration of one likely determinant of contact patterns in this paper, namely the preference for sexual partners with one's own frequency of sexual contacts, demonstrates that actual contact patterns are likely to have a dramatic effect on the course of the epidemic. This is consistent with the results of Hyman and Stanley [70]. Other factors that will create other influential patterns of contact no doubt include geography, sexual preference (especially bisexuality), prostitution, needle sharing, and age.

Epidemiology has not developed a discipline devoted to examining the effects of contact patterns in populations on the behavior of transmission in those populations. Little has been done to postulate the effects of contact patterns or to collect data relevant to this issue. John Fox, Lila Elveback, and Gene Ackerman began an effort to examine contact pattern effects using Monte Carlo simulation models; the results of that cndeavor have been summarized [88]. No large body of investigators is pursuing this line of work, but the original model continues to be elaborated by Ackerman and his colleagues.

The deterministic differential equation modeling of infection transmission has developed a broader body of investigators following its traditions than has Monte Carlo simulation of transmission. Until recently, heterogeneities of populations were not a major focus of this tradition. Now much more attention is being paid to the effects of heterogeneities in populations [51-58]. Almost all AIDS models have some population heterogeneities [67-72]. However, all published models have assumed random mixing between heterogeneous groups. We have shown that the effects of the assumptions of heterogeneity are likely to be quite important and are likely to complicate some of the aspects of transmission dynamics that might be most important to us.

As the AIDS epidemic progresses and our ability to control it improves with new therapeutic agents and vaccines, many questions about setting priorities for different types of programs in different types of groups will arise. Are there key contact groups in which we must employ expensive direct contact methods to ensure that our educational efforts are successful? Which population groups deserve special case-finding methods to ensure that we find all infected individuals and offer them the therapy or education at our disposal? Which groups will require $90 \%, 99 \%$, or higher vaccination rates in order to significantly reduce overall population infection rates? 
These questions could be addressed by second-stage models that build on the models presented here. However, to do so, not only are more models needed but better data are needed to estimate parameters. Key parameters besides the contact rates are the transmission probabilities at different stages of infection. Techniques have recently been developed by Longini et al. [60-62] that enable one to estimate transmission probabilities as multivariate functions of risk factors from observational data that can be cross-sectional. The use of these techniques, combined with investigational efforts to determine just what the real mixing patterns are, could begin to form the basis of a science examining the effects of contact patterns in populations on HIV transmission. The demonstration here of the dramatic effects of contact patterns on transmission dynamics attests to the need for such a development.

\section{APPENDIX A. MATHEMATICAL PROPERTIES OF MODEL EQUATIONS}

The most studied system of differential equations in mathematical epidemiology is the system of $3 M$ equations:

$$
\begin{aligned}
& \dot{X}_{i}=b_{i}\left(N_{i}-X_{i}\right)-X_{i} \sum_{j} \lambda_{i j} Y_{j}+\kappa_{i} Z_{i} \\
& \dot{Y}_{i}=-\gamma_{i} Y_{i}-b_{i} Y_{i}+X_{i} \sum_{j} \lambda_{i j} Y_{j} \\
& \dot{Z}_{i}=-b_{i} Z_{i}-\kappa_{i} Z_{i}+\gamma_{i} Y_{i},
\end{aligned}
$$

for $i=1, \ldots, M$. In this model, $X_{i}, Y_{i}$, and $Z_{i}$ denote the number of susceptible, infected, and removed individuals, respectively, in subpopulation $i$, and $N_{i}$ denotes the total size of population $i$, which is assumed to be constant. Here, $b_{i}$ is the birth and death rate of subpopulation $i, \lambda_{i j}$ is the effective contact rate between individuals in group $i$ and infectives in group $j, \gamma_{i}$ is the recovery rate for group $i$, and $\kappa_{i}$ is the rate at which recovered individuals lose their immunity. All parameters are nonnegative. This system includes the general SI, SIS, SIR, and SIRS models that are used to study the spread of contagious diseases.

System (A1) has bcen well-studied and some of its important properties have been demonstrated $[46,58,63,89-91]$.

(1) There is a compact, convex set $B$ in $\mathbf{R}_{+}^{3 M}$ such that all solutions of (A1) that start in $B$ remain in $B$.

(2) $N_{i}=X_{i}+Y_{i}+Z_{i}$ is a conserved quantity; that is, it is constant along solutions of (A1). 
(3) The no-disease equilibrium, $X_{i}=N_{i}, Y_{i}=Z_{i}=0$, is always a rest point for this system.

(4) There is a function $G\left(\gamma_{i}, \lambda_{i j}, \kappa_{i}, b_{i}\right)$ of the parameters of the system (A1), called the threshold, with the property that when $G<1$, the no-disease equilibrium is locally asymptotically stable, and when $G>1$, it is unstable.

(5) When $G<1$, the no-disease equilibrium is the only equilibrium, and it is globally asymptotically stable.

(6) When $G>1$, a unique new equilibrium $\mathbf{X}^{*}$, the endemic equilibrium, arises in $B$; it is locally asymptotically stable.

(7) For SI and SIS models, $\mathbf{X}^{*}$ is globally asymptotically stable in $B-\partial B$. (Whether this is true in SIR and SIRS models is a major unsolved problem in mathematical epidemiology.)

The system (1)-(4) of differential equations that we use to model the spread of the HIV virus differs in three fundamental ways from the generic system (A1):

(1) The interaction terms are no longer quadratic but are the rational functions with variable denominators

$$
\frac{X_{i} Y_{j}}{X_{j}+Y_{j}}
$$

(2) The model incorporates different stages of infectivity with different degrees of contagion.

(3) The contact fractions $\rho_{i j}$ may depend on the sizes of the various subgroups and thus could vary over time.

From our many simulations, it appears that properties 1-7 still hold for our more complex system. Proving all these properties mathematically seems like a herculean task. In this appendix, we describe the progress we have made on this program.

PROPERTY 1a

For $\mu>0$, let $N_{i}=X_{i}+Y_{i 1}+\cdots+Y_{i m}+Z_{i}$. If $N_{i}(0) \leqslant U_{i} / \mu$, then $N_{i}(t)$ $\leqslant U_{i} / \mu$ for all $t$. In particular, the box

$$
\begin{array}{r}
C \equiv\left\{\left(X_{1}, \ldots, Z_{M}\right): X_{i}+Y_{i 1}+\cdots+Z_{i} \leqslant U_{i} / \mu,\right. \\
\left.X_{i} \geqslant 0, Y_{i r} \geqslant 0, Z_{i r} \geqslant 0 \text { for all } i, r\right\}
\end{array}
$$

is a compact, convex invariant set in $\mathbf{R}_{+}^{3 M}$.

Proof. By summing Equations (1)-(4), one finds

$$
\dot{N}_{i}=-\mu N_{i}+U_{i}-(\delta-\mu) Z_{i},
$$


where $\delta-\mu>0$. Multiply the inequality

$$
\dot{N}_{i}+\mu N_{i} \leqslant U_{i}
$$

by $e^{\mu t}$ and integrate from 0 to $T$ to prove that if $N_{i}(0) \leqslant U_{i} / \mu$, then $N_{i}(T) \leqslant U_{i} / \mu$ for all $T$.

To finish showing that $C$ is invariant, we must show that solutions that start on $X_{i}=0$ or $Y_{i r}=0$ boundaries of $C$ move into $C$. This is straightforward. For example, when $X_{i}=0, \dot{X}_{i}=U_{i}>0$, and orbits move into $C$; when $Y_{i r}=0, \dot{Y}_{i r} \geqslant 0$, and orbits move into $C$.

PROPERTY $1 \mathrm{~b}$

For $\mu=0$, the solution with initial conditions $X_{i}(0)=X_{i}^{0}>0, Y_{i r}(0)=$ $0, Z_{i}(0)=0$ is $X_{i}(t)=U_{i} t+X_{i}^{0}, Y_{i r}(t)=Z_{i}(t)=0$, which tends to infinity as $t \rightarrow \infty$.

\section{PROPERTY 2}

$N_{i}$ is no longer conserved. For example, if $\delta=\mu$ in (A2), $N_{i}$ satisfies $N_{i}(t)=N_{i 0} e^{-\mu t}+U_{i} / \mu$, which varies with $t$ and tends to $U_{i} / \mu$.

\section{PROPERTY 3}

For $\mu>0$,

$$
X_{i}=U_{i} / \mu, \quad Y_{i 1}=\cdots=Y_{i m}=Z_{i}=0
$$

is the no-disease equilibrium. For $\mu=0$, the corresponding state is

$$
X_{i}=\infty, \quad Y_{i 1}=\cdots=Y_{i m}=Z_{i}=0 .
$$

\section{PROPERTIES 4,5,6,7}

The calculations behind Tables 1 and 2 in Section 7 and Appendix B compute the existence of a threshold with the property that when the parameters of (1)-(4) lie below this threshold, the no-disease equilibrium is the only equilibrium; and when this threshold is exceeded, a unique new endemic equilibrium appears. These endemic equilibria are listed in Tables 1 and 2. We have not yet completely tied this threshold to the stability characteristics of the two equilibria, as has been accomplished for the simpler system (A1). We now present some calculations with simplifications of our model that preserve some of its unique features. These calculations illustrate that this threshold is related to the change in stability of the no-disease equilibrium that occurs for system (A1). Our hundreds of simulations of (1)-(4) with different parameter value thoroughly support this link. They also suggest that the endemic equilibrium is globally asymptotically stable in our model, as has been proved for the SI model by Lajmanovich and Yorke [63] and conjectured for the SIR model by a number of people. 
Two special features of our model are the role of death from the disease and the multiplicity of stages of the disease. We will study two generalizations of our system (1)-(4) that bring these complications in one at a time. To keep the mathematics tractable, we will work only with restricted mixing at this first stage. In this case, there is no interaction between subpopulations, so we can study a single population without loss of generality.

We first bring deaths into the study. The equations for the spread of the disease become

$$
\dot{X}=-\frac{\lambda X Y}{X+Y}-\mu X+U, \quad \dot{Y}=\frac{\lambda X Y}{X+Y}-(k+\mu) Y, \quad \dot{Z}=k Y-\delta Z .
$$

Since the first two equations are independent of $Z$, we can work with just these two, without loss of generality. We first consider the case $\mu=0$ :

$$
\dot{X}=-\frac{\lambda X Y}{X+Y}+U, \quad \dot{Y}=\frac{\lambda X Y}{X+Y}-k Y .
$$

The $\{\dot{X}=0\}$ isocline is the curve $Y=U X /(\lambda X-U)$; the $\{\dot{Y}=0\}$ isoclines are the lines

$$
Y=0 \quad \text { and } \quad Y=\frac{\lambda-k}{k} X .
$$

There are two cases depending on the sign of $\lambda-k$. If $\lambda-k<0$, the line $Y=[(\lambda-k) / k] X$ misses the positive quadrant and the isoclines intersect as in Figure 20. In this case, as can be seen in Figure 20, all orbits tend to $X=\infty, Y=0$, amplifying the statement in Property $1 \mathrm{~b}$. All orbits are monotonically decreasing in $Y$, so $V(X, Y)=Y$ is a Lyapunov function for this system.

When $\lambda-k>0$, the isoclines are as in Figure 21. Note that the threshold $\lambda-k$ appears as the denominator of the $X$ value of the endemic equilibrium, just as it does for our calculations of the threshold that are summarized in Tables 1 and 2 . Furthermore, one can prove directly that the Jacobian derivative of system (A4) has a positive determinant and a negative trace so that both of its eigenvalues have negative real part. This means that the endemic equilibrium is locally asymptotically stable, when it exists.

To prove that it is globally asymptotically stable, we use the BendixsonduLac criterion to rule out the existence of periodic orbits (see [92] or [93]). First, multiply both equations of (A4) through by $1 / Y$. This will change the length of the vector field of (A4) but not the direction of the vectors and therefore not the phase portrait. The new system is

$$
\dot{X}=-\frac{\lambda X}{X+Y}+\frac{U}{Y}, \quad \dot{Y}=\frac{\lambda X}{X \mid Y}-k .
$$




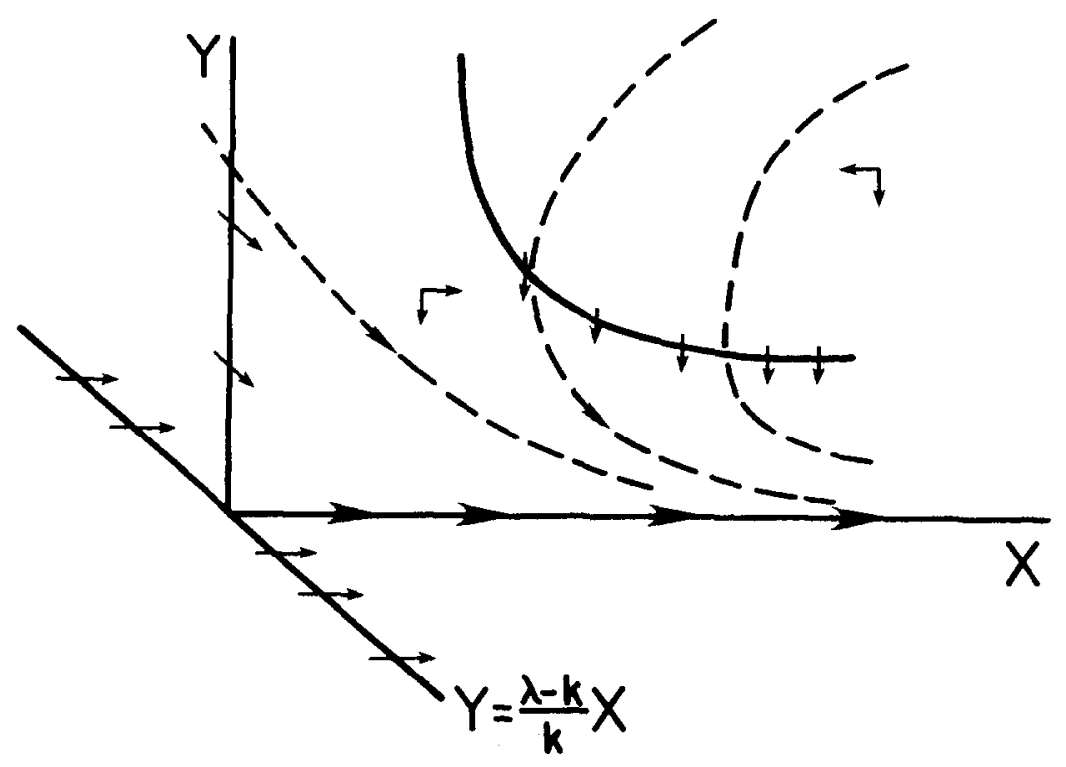

FIG. 20. Phase diagram for simplified system, $\mu=0$ and $\lambda-k<0$.

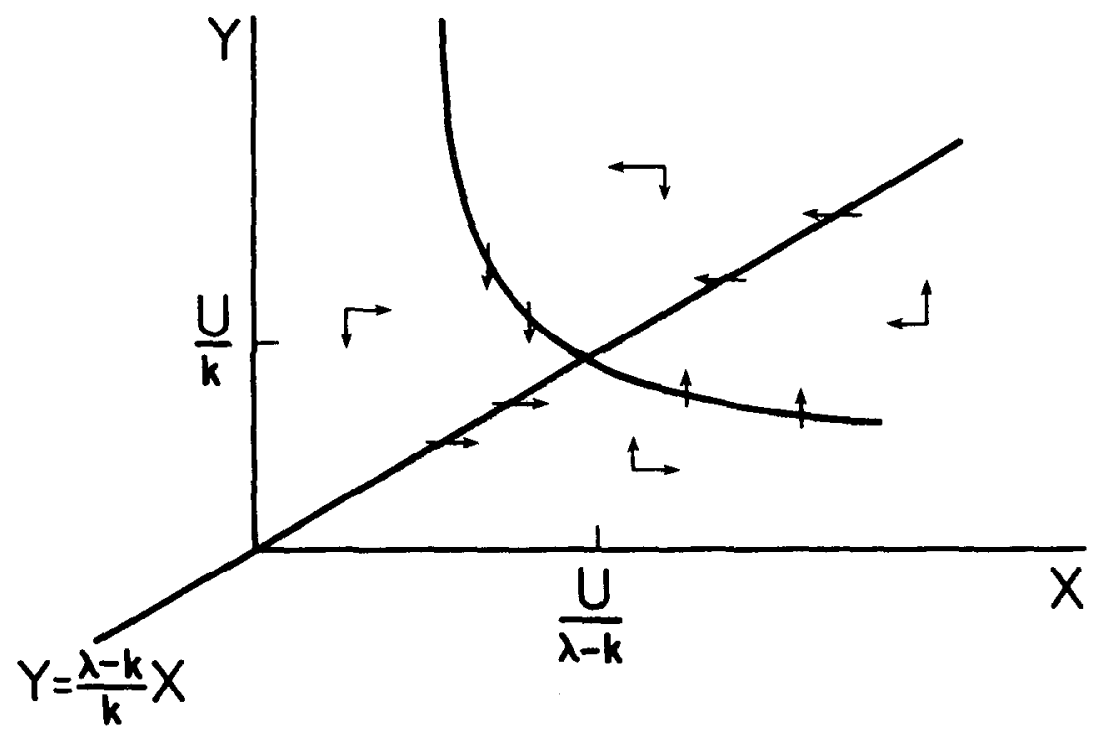

FIG. 21. Phase diagram for simplified system, $\mu=0$ and $\lambda-k>0$. 
The trace of the Jacobian of this system is

$$
\frac{\partial \dot{X}}{\partial X}+\frac{\partial \dot{Y}}{\partial Y}=-\frac{\lambda Y}{(X+Y)^{2}}-\frac{\lambda X}{(X+Y)^{2}}=-\frac{\lambda}{X+Y},
$$

which is negative for all $(X, Y)$. But this is precisely the Bendixson-duLac criterion, which rules out the existence of periodic orbits in Figure 21 and guarantees, by the Poincaré-Bendixson theorem, that all orbits must tend to the endemic equilibrium. Alternatively, one can use Olech's theorem [94] and similar calculations to prove directly that the endemic equilibrium is globally asymptotically stablc.

We next turn to the $\mu>0$ case, system (A3). The $\{\dot{X}=0\}$ isoclines are the curves

$$
Y=\frac{X(U-\mu X)}{(\lambda+\mu) X-U}
$$

The $\{\dot{Y}=0\}$ isoclines are the lines

$$
Y=0 \quad \text { and } \quad Y=\frac{\lambda-(k+\mu)}{k+\mu} X .
$$

Once again, there are two phase portraits, depending on whether or not $\lambda-(k+\mu)$ is negative or positive.

Case 1. $\lambda-(k+\mu)<0$. The isoclines for this case are drawn in Figure 2. It is clear that the no-disease equilibrium is the only nonzero steady state, that it is globally asymptotically stable, and that $V(X, Y)=Y$ is a Lyapunov function, i.e., that the number of infecteds decreases monotonically to zern along any solution curve.

Case 2. $\lambda-(k+\mu)>0$. The isoclines for this case are drawn in Figure 3. As the threshold $\lambda-(k+\mu)$ becomes positive, the denominator of the $Y$ coordinate of the other equilibrium becomes positive, and the no-disease equilibrium becomes unstable. One can easily use the Jacobian at the endemic equilibrium to show that this equilibrium is locally asymptotically stable when it exists. Furthermore, one uses the Bendixson-duLac criterion or Olech's theorem exactly as in the $\mu=0$ case to prove that the endemic equilibrium is globally asymptotically stable, when it exists.

\section{CONCLUSION}

For $\mu>0$, the seven conclusions of the generic SIR system (A1) still hold for equations (A3), which model the spread of a disease in which death plays a role, but with restricted mixing and a single stage of infection. 
Finally, we expand this model by allowing multiple stages of the disease:

$$
\begin{aligned}
\dot{X} & =-c X \frac{\sum_{j} \beta_{j} Y_{j}}{X+Y}-\mu X+U \\
\dot{Y} & =c X \frac{\sum_{j} \beta_{j} Y_{j}}{X+Y}-(k+\mu) Y_{1} \\
\dot{Y}_{j} & =k Y_{j-1}-(k+\mu) Y_{j}, \quad \text { for } j=2, \ldots, m .
\end{aligned}
$$

Recall that our model (1)-(4) for the spread of HIV has $m$ stages of disease. The no-disease equilibrium is $X=U / \mu, Y_{j}=0$ for $j=1, \ldots, m$. Use the change of coordinates $S=U / \mu-X$ or $X=U / \mu-S$ to move the no-disease equilibrium to the origin. In these new $\left(S, Y_{1}, \ldots, Y_{m}\right)$ coordinates, system (A5) becomes

$$
\begin{aligned}
& \dot{S}=\frac{c X}{X+Y} \sum_{j} \beta_{j} Y_{j}-\mu S \\
& \dot{Y}_{1}=\frac{c X}{X+Y} \sum_{j} \beta_{j} Y_{j}-(k+\mu) Y_{1} \\
& \dot{Y}_{j}=k Y_{j-1}-(k+\mu) Y_{j}, \quad j=2, \ldots, m .
\end{aligned}
$$

Use

$$
\frac{X}{X+Y}=1-\frac{Y}{X+Y}
$$

to expand the right-hand side of (A6) into a Taylor series of order one. Then write this system in matrix form as

$$
\begin{aligned}
\left(\begin{array}{c}
\dot{S} \\
\dot{Y}_{1} \\
\dot{Y}_{2} \\
\vdots \\
\dot{Y}_{m}
\end{array}\right)= & \left(\begin{array}{cccccc}
-\mu & c \beta_{1} & c \beta_{2} & \cdots & c \beta_{m-1} & c \beta_{m} \\
0 & c \beta_{1}-(k+\mu) & c \beta_{2} & \cdots & c \beta_{m-1} & c \beta_{m} \\
0 & k & -(k+\mu) & \cdots & 0 & 0 \\
\vdots & \vdots & \vdots & \ddots & \vdots & \vdots \\
0 & 0 & 0 & \cdots & k & -(k+\mu)
\end{array}\right) \\
& \times\left(\begin{array}{c}
S \\
Y_{1} \\
Y_{2} \\
\vdots \\
Y_{m}
\end{array}\right)+\left(\begin{array}{c}
-\frac{c Y}{X+Y} \sum_{j} \beta_{j} Y_{j} \\
-\frac{c Y}{X+Y} \sum_{j} \beta_{j} Y_{j} \\
0 \\
\vdots \\
0
\end{array}\right) .
\end{aligned}
$$


Letting $\mathbf{x} \equiv\left(S, Y_{1}, \ldots, Y_{m}\right)^{T}$, we can write this system as

$$
\ddot{\mathbf{x}}=A \mathbf{x}+Q(\mathbf{x}),
$$

where

(1) $A=\left(\begin{array}{cc}-\mu & * \\ 0 & A_{1}\end{array}\right)$

(2) $A_{1}$ is a Metzler matrix in that all off-diagonal entries are nonnegative; in fact, $A$ and $A_{1}$ are compartmental matrices,

(3) $Q(\mathbf{x})=o(\mathbf{x})$, i.e., $Q(\mathbf{x}) /\|\mathbf{x}\| \rightarrow \mathbf{0}$ as $\mathbf{x} \rightarrow \mathbf{0}$, and

(4) $Q(\mathbf{x}) \leqslant 0$ for all $\mathbf{x}$ in the domain of definition.

We use the proof in [63] as modified in [58] to prove that when $\mathbf{x}=\mathbf{0}$ is locally stable, it is globally stable. The threshold is the Pcrron-Frobenius eigenvalue of the Metzler matrix $A_{1}$. We will just sketch the proof here; the details can be found in the referenced works.

We assume that all the $c, \beta_{i}, k$ are strictly positive. This implies that $A_{1}$ is irreducible, i.e., that some power of $A_{1}$ has no zero entries. Because $A_{1}$ is an irreducible Metzler matrix, so is its transpose $A_{1}^{T}$. By the Perron-Frobenius theorem, $A_{1}^{T}$ has a unique eigenvalue $r$ of largest real part; this eigenvalue $r$ is real, has algebraic and geometric multiplicity 1 , and has an eigenvector $\mathbf{v}$ in $\mathbf{R}^{m}$ with only positive components. When $r<0$, then $A_{1}^{T}, A_{1}$, and $A$ have only eigenvalues with negative real part. In this case, $\mathbf{x}=\mathbf{0}$ is locally asymptotically stable. To show that it is globally asymptotically stable, use the Lyapunov function

$$
V(S, \mathbf{Y})=(0, \mathbf{v})^{T}(S, \mathbf{Y})=\mathbf{v}^{T} \mathbf{Y}
$$

For

$$
\begin{aligned}
\dot{V}(S, \mathbf{Y}) & =\mathbf{v}^{T} A_{1} \mathbf{Y}+(0, \mathbf{v}) \cdot Q(S, \mathbf{Y}) \\
& =r \mathbf{v}^{T} \mathbf{Y}+(0, \mathbf{v}) \cdot Q(S, \mathbf{Y}) \\
& \leqslant 0,
\end{aligned}
$$

since $\mathbf{v}>0, \mathbf{Y} \geqslant \mathbf{0}, Q \leqslant \mathbf{0}$, and $r<0$. Furthermore, the set $\{V=0\}$ is the $\{\mathbf{Y}=\mathbf{0}\}$ subspace on which the system (A6) tends globally to $(S, \mathbf{Y})=(0, \mathbf{0})$. These statements imply that all orbits of (A6) tend to the no-disease equilibrium.

When $r>0$, the origin becomes unstable, and most orbits tend away from 0 . The argument in [58] shows that a new equilibrium must exist inside $C$. The computations in Section 7 and Appendix B show that this endemic equilibrium is unique. We prove now that it is locally asymptotically stable and conjecture that it is globally asymptotically stable. 
For simplicity of notation, we will prove local stability of the endemic equilibrium for the $\mu=0$ case. This automatically implies local stability for $\mu>0$ and small enough. However, a similar proof works for general $\mu>0$.

Also, for simplicity of notation, we will treat $m=2$. The generalization to $m>2$ is straightforward. In this case, system (A5) becomes

$$
\begin{aligned}
& \dot{X}=-\frac{c X}{X+Y}\left[\beta_{1} Y_{1}+\beta_{2} Y_{2}\right]+U, \\
& \dot{Y}_{1}=\frac{c X}{X+Y}\left[\beta_{1} Y_{1}+\beta_{2} Y_{2}\right]-k Y_{1}, \\
& \dot{Y}_{2}=k Y_{1}-k Y_{2},
\end{aligned}
$$

where $Y=Y_{1}+Y_{2}$. As we show in Section 7, the unique endemic equilibrium is

$$
\left(X, Y_{1}, Y_{2}\right)=\frac{m U}{k}\left(\frac{k}{c \bar{\beta} m-k}, 1,1\right) .
$$

The threshold condition for an endemic equilibrium is

$$
\frac{c \bar{\beta} m}{k}>1
$$

The Jacobian derivative of (A7) at the endemic equilibrium (A8) is

$$
\mathrm{DF}=\left(\begin{array}{ccc}
-\frac{U Y}{X(X+Y)} & \frac{U-\beta_{1} c X}{X+Y} & \frac{U-\beta_{2} c X}{X+Y} \\
\frac{U Y}{X(X+Y)} & \frac{\beta_{1} c X-U}{X+Y}-k & \frac{\beta_{2} c X-U}{X+Y} \\
0 & k & -k
\end{array}\right)
$$

The characteristic polynomial of DF is

$$
\lambda^{3}+a_{1} \lambda^{2}+a_{2} \lambda+a_{3}=0,
$$

where $a_{i}=(-1)^{i+1}$ times the sum of the $i \times i$ principal minors of DF. We show that each $a_{i}$ is positive. Then, by Descartes' rule of signs, every root of (A10) must be negative or have negative real part. It follows that the endemic equilibrium (A8) is locally asymptotically stable.

In the first place, $a_{1}$ is the negative of the trace of DF. The $(1,1)$ and $(3,3)$ terms of DF are clearly negative. Use (A9) to write the $(2,2)$ entry as

$$
\frac{\beta_{1} c X-U}{\bar{X}+Y}-k=\frac{k}{m c \bar{\beta} m}\left(m c\left[\beta_{1}-m \bar{\beta}\right]+[k-c \bar{\beta} m]\right) \text {. }
$$


The first square-bracketed factor in (A11) is negative since $m \bar{\beta}=\sum \beta_{i}$; the second is negative by the threshold condition (A9). Therefore $a_{1}$ is positive.

The coefficient $a_{2}$ in (A10) is the sum of the three principal $2 \times 2$ minors DF. The $(1,3)$ minor is positive by inspection. The $(1,2)$ minor is positive by a straightforward calculation. By a longer calculation, the $(2,3)$ minor equals

$$
\frac{2 k^{2}}{m^{2} c \bar{\beta}}(c \bar{\beta} m-k) \text {, }
$$

which is positive by (A9). Therefore, $a_{2}$ is positive.

Finally, since $a_{3}$ is the negative of the determinant of DF, we need only compute this determinant. This calculation is made simpler by first adding row 1 to row 2 and then adding the new row 2 to row 3, operations that do not change the determinant. The resulting matrix is upper triangular (row echelon form), and its determinant is easily seen to be $-k^{2} U Y / X(X+Y)$. Since this number is negative, $a_{3}$ is positive. Therefore, all the coefficients of (A10) are positive, and so all its roots are negative or have negative real part -a sufficient condition for the local stability of the endemic equilibrium.

\section{APPENDIX B. CALCULATIONS FOR THE PREFERRED MIXING EQUILIBRIUM AND THRESHOLD FOR $\mu=0$}

In this appendix, we will prove the uniqueness of the equilibrium for the preferred mixing case with $\mu=0$, and we will derive the threshold condition. In the process, we will show why the iteration procedure described after Equation (36) works so well.

After plugging $Y_{i}^{s}=U_{i} m / k$ into Equation (34), we can consider (34) and (35) as $n+1$ equations in the $n+1$ unknowns $X_{1}^{s}, \ldots, X_{n}^{s}, K^{s}$. In this appendix we drop the superscript $s$ and use $K$ for $K_{Y}^{s}$. It is natural to construct a mapping

$$
K \mapsto T(K)
$$

from $[0,1]$ to $[0,1]$ as follows:

For any choice of $K \in[0,1]$, (36) determines a mapping

$$
K \mapsto X_{i}(K)
$$

for $i=1, \ldots, n$. Furthermore, (34) defines a mapping

$$
\left(X_{1}, \ldots, X_{n}\right) \rightarrow K=G\left(X_{1}, \ldots, X_{n}\right) .
$$

Let

$$
T(K)=G\left(X_{1}(K), \ldots, X_{n}(K)\right)
$$


Since the range of $G$ is $[0,1]$, the range of $T$ is naturally $[0,1]$. Then, $X_{1}, \ldots, X_{n}, K$ is a solution of the $n+1$ equations (34) and (36) if and only if $K=T(K)$ and $X_{i}=F_{i}(K)$ for $i=1, \ldots, n$. Therefore, the fixed points of $T$ correspond to the endemic equilibria of our system. Of course, the maps $X_{i}$, $G$, and $T$ depend on the parameters $U, \mathbf{c}, \rho, \bar{\beta}$.

To study the fixed points of $T$, we write out the map $T$ explicitly by plugging $Y_{i}=U_{i} m / k$ and the expression for $X_{i}(K)$ from (36) into the definition (34) of $K$ :

$$
\begin{aligned}
T(K) & =\frac{(m / k) \sum c_{j}\left(1-\rho_{j}\right) U_{j}}{(1 / 2 \bar{\beta} K) \Sigma U_{j}\left(-P_{j}+\sqrt{P_{j}^{2}+Q_{j}}\right)+(m / k) \sum c_{j}\left(1-\rho_{j}\right) U_{j}} \\
& =\frac{1}{(1 / 2 \bar{\beta} \hat{U} K) \Sigma U_{j}\left(-P_{j}+\sqrt{P_{j}^{2}+Q_{j}}\right)+1}
\end{aligned}
$$

where $\hat{U} \equiv(m / k) \sum_{j} c_{j}\left(1-\rho_{j}\right) U_{j}$. Note that $T(0)=0$. However, by (34), this fixed point corresponds to some $X_{j}^{s}$ being infinite. Since we are looking for a finite endemic steady state, we need a strictly positive fixed point of $T$. Using (B1), we can write the fixed point equation $T(K)=K$ as

$$
\frac{K}{(1 / 2 \bar{\beta} \hat{U}) \Sigma U_{j}\left(-P_{j}+\sqrt{P_{j}^{2}+Q_{j}}\right)+K}=K,
$$

which can be rearranged to

$$
\frac{1}{2 \bar{\beta} \hat{U}} \sum U_{j}\left(-P_{j}+\sqrt{P_{j}^{2}+Q_{j}}\right)=1-K .
$$

The graph of $K \mapsto 1-K$ is the negatively sloped line in Figure 22. We will show that the graph of the function on the left-hand side of Equation (B2) is a positively sloped curve. This implies that if it starts below the graph of $K \mapsto 1-K$, it must cross it exactly once; if it starts above the graph, it won't cross it at all. This naturally leads to the uniqueness of the endemic equilibrium and to the threshold condition!

To prove that the function on the left-hand side of (B2) is an increasing function of $K$, we need only prove that each

$$
S_{j}(K) \equiv-P_{j}(K)+\left[P_{j}(K)^{2}+Q_{j}(K)\right]^{1 / 2}
$$

is an increasing function of $K$. We first observe that

$$
0 \leqslant S_{i} \leqslant 2 \text {. }
$$




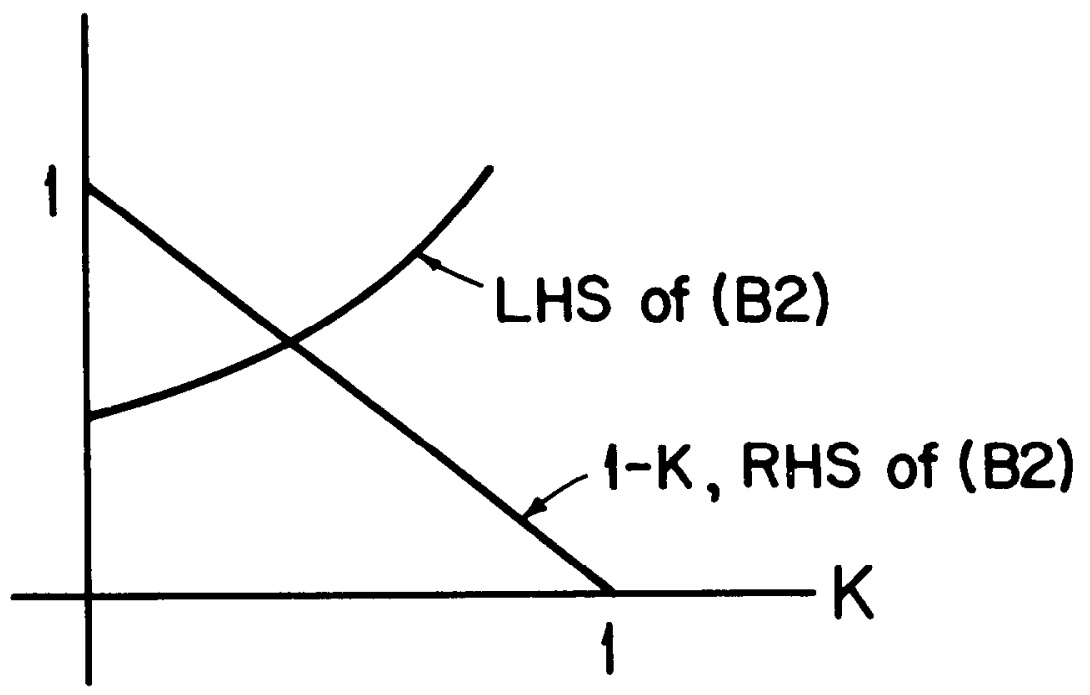

FIG. 22. Equation (B2).

For, dropping the subscript $j$ for ease of notation, we compute

$$
\begin{aligned}
S & =1-b((1-\rho) K+\rho)+\left\{[b((1-\rho) K+\rho)-1]^{2}+4 b(1-\rho) K\right\}^{1 / 2} \\
& \leqslant 1-b((1-\rho) K+\rho)+\left\{[b((1-\rho) K+\rho)-1]^{2}+4 b(1-\rho) K+4 b p\right\}^{1 / 2} \\
& =1-b((1-\rho) K+\rho)+\left\{[b((1-\rho) K+\rho)+1]^{2}\right\}^{1 / 2} \\
& =2
\end{aligned}
$$

where $b \equiv \bar{\beta} c_{i}(m / k)$.

We next prove that $\partial S / \partial K>0$. Note that $S+P=\sqrt{P^{2}+Q}$. Squaring both sides leads to the equation

$$
S^{2}+2 S P=Q
$$

Letting a prime denote the derivative with respect to $K$, we find

$$
2 S S^{\prime}+2 S^{\prime} P+2 S P^{\prime}=Q^{\prime} \quad \text { or } \quad S^{\prime}=\frac{Q^{\prime}-2 S P^{\prime}}{2(S+P)}
$$

The denominator in (B4) is positive since $S+P=\sqrt{P^{2}+Q}$. By the defini- 
tions of $P$ and $Q$ in (36),

$$
Q^{\prime}=4 b(1-\rho)=4 P^{\prime}
$$

Substituting this into the numerator of (B4), we find

$$
Q^{\prime}-2 S P^{\prime}=4 P^{\prime}-2 S P^{\prime}=2 P^{\prime}(2-S)
$$

Since $P^{\prime} \geqslant 0$ by (B5) and $2-S \geqslant 0$ by (B3), the numerator of (B4) is nonnegative. Therefore, each $S_{j}(K)$ is a nondecreasing function of $K$, and the left-hand side of Equation (B2) is a nondecreasing function of $K$. If this left-hand side is less than or equal to 1 at $K=0$, as in Figure 22, then the two graphs must cross and $T$ has a nonzero fixed point, positive or zero; if the left-hand side is greater than 1 at $K=0, T$ cannot have a nonnegative fixed point. It follows that the value of the left-hand side of (B2) at $K=0$ gives the threshold for the preferred mixing case:

$$
\begin{aligned}
\tau & \equiv \frac{\sum_{j} U_{j}\left[1-\bar{\beta} c_{j}(m / k) \rho_{j}+\left|1-\bar{\beta} c_{j}(m / k) \rho_{j}\right|\right]}{2 \bar{\beta}(m / k) \Sigma_{j} c_{j}\left(1-\rho_{j}\right) U_{j}} \\
& =\frac{\sum_{j} \max \left\{0,1-\bar{\beta} c_{j}(m / k) \rho_{j}\right\} U_{j}}{\bar{\beta}(m / k) \sum_{j} c_{j}\left(1-\rho_{j}\right) U_{j}}
\end{aligned}
$$

If $\tau \leqslant 1$, then there will be a unique endemic equilibrium; if $\tau>1$, then there is no endemic equilibrium.

The reader can easily check that this result simplifies to our earlier result for proportional mixing, by setting each $\rho_{j}$ to zero. On the other hand, for the restricted mixing case $\left(\rho_{i}=1\right.$ for all $i$ ), the denominator of $\tau$ is zero. Unless the numerator is zero also, we can consider $\tau>1$. Then, the only way for $\tau$ to be less than 1 is if

$$
1-\bar{\beta} c_{j}\left(\frac{m}{k}\right) \leqslant 0 \quad \text { or } \quad \bar{\beta} \geqslant \frac{1}{c_{j} m / k}
$$

for all $j$. But this is the thrcshold condition we derived in Section 7 for restricted mixing.

If $1-\bar{\beta} c_{j}(m / k) \rho_{j}>0$ for all $j$, the threshold condition reduces to

$$
\frac{\sum_{j}\left[1-\bar{\beta}(m / k) c_{j} \rho_{j}\right] U_{j}}{\bar{\beta}(m / k) \sum_{j} c_{j}\left(1-\rho_{j}\right) U_{j}}<1
$$


which simplifies to

$$
1<\frac{\bar{\beta}(m / k) \sum_{j} c_{j} U_{j}}{\sum_{j} U_{j}}=\bar{\beta}(m / k) \bar{c}_{U} .
$$

Condition (B7) gives, as the threshold for $\bar{\beta}$;

$$
\bar{\beta}_{T}=\frac{1}{(m / k) \bar{c}_{U}},
$$

which is the same as the threshold found for proportional mixing in Section 7 .

APPENDIX C. STEADY STATES FOR $\mu>0$

EQUATIONS FOR STEADY STATES

For steady states characterized by $\dot{X}_{i}=0, \dot{Y}_{i r}=0$, Equations (1)-(3) become

$$
\begin{aligned}
& U_{i}=c_{i} X_{i}^{s} \sum_{j} \rho_{i j} \sum_{j} \beta_{i j r}\left(\frac{Y_{j r}^{s}}{X_{j}^{s}+Y_{j}^{s}}\right)+\mu X_{i}^{s} \\
& Y_{i 1}^{s}=\frac{c_{i} X_{i}^{s}}{(k+\mu)} \sum_{j} \rho_{i j} \sum_{r} \beta_{i j r}\left(\frac{Y_{j r}^{s}}{X_{j}^{s}+Y_{j}^{s}}\right)=\frac{U_{i}-\mu X_{i}^{s}}{(k+\mu)} \\
& Y_{i r}^{s}=\frac{k^{r-1}}{(k+\mu)^{r}}\left(U_{i}-\mu X_{i}^{s}\right) ; \quad r=2, \ldots, m \\
& Y_{i}^{s} \equiv \sum_{r=1}^{m} Y_{i r}^{s}=\sum_{r=1}^{m} \frac{k^{r \cdots 1}}{(k+\mu)^{r}}\left(U_{i}-\mu X_{i}^{s}\right)=\frac{1}{\mu}\left[1-\left(\frac{k}{k+\mu}\right)^{m}\right]\left(U_{i}-\mu X_{i}^{s}\right)
\end{aligned}
$$

NOTATION

The two factors in (C4) appear frequently in this appendix, so we develop special notation for them. Let $\alpha \equiv k /(k+\mu)$. The term $\left(1-\alpha^{m}\right)$ can be written in the form

$$
\left(1-\alpha^{m}\right)=1-\frac{k^{m}}{(k+\mu)^{m}}=\frac{m k^{m-1} \mu}{(k+\mu)^{m}}\left[1+g\left(\frac{\mu}{k}\right)\right],
$$

in which $g(\mu / k)$ is given by

$$
g\left(\frac{\mu}{k}\right)=\frac{m-1}{2}\left(\frac{\mu}{k}\right)+\frac{(m-1)(m-2)}{3 !}\left(\frac{\mu}{k}\right)^{2}+\cdots+\frac{1}{m}\left(\frac{\mu}{k}\right)^{m-1} .
$$

Equation (C5) is useful because

$$
g\left(\frac{\mu}{k}\right) \rightarrow 0 \quad \text { and } \quad \frac{1-\alpha^{m}}{\mu} \rightarrow \frac{m}{k} \quad \text { as } \mu \rightarrow 0 .
$$


We can use these facts to compare our results for $\mu>0$ with those for $\mu=0$. In our calculations in this appendix, we will write $\theta_{\mu}$ for $\left(1-\alpha^{m}\right) / \mu$. Furthermore, define $W_{i}$ by

$$
W_{i} \equiv U_{i}-\mu X_{i}^{s}
$$

Then (C4) becomes

$$
Y_{i}^{s}=\theta_{\mu} W_{i}
$$

THE STEADY STATE DEPENDS ON A WEIGHTED MEAN OF THE $\beta_{i, r}$

Following the same path as in Section 7, we use (C3) and (C4) to derive the generalization (C7) of (17):

$$
\begin{aligned}
\sum_{r} \beta_{i j r} \frac{Y_{j r}^{s}}{X_{j}^{s}+Y_{j}^{s}} & =\frac{Y_{j}^{s}}{X_{j}^{s}+Y_{j}^{s}} \sum_{r} \frac{k^{r-1} /(k+\mu)^{r}}{\frac{1}{\mu}\left[1-\left(\frac{k}{k+\mu}\right)^{m}\right]} \beta_{i j r} \\
& =\frac{Y_{j}^{s}}{X_{j}^{s}+Y_{j}^{s}} \sum_{r=1}^{m} \frac{1}{m\left(\frac{k}{k+\mu}\right)^{m-r}\left[1+g\left(\frac{\mu}{k}\right)\right]} \beta_{i j r}
\end{aligned}
$$

The coefficients of $\beta_{i j r}$ are $Y_{j r}^{s} / Y_{j}^{s}$ and add to 1 ; so the summation over $r$ again gives a mean transmission fraction, but a weighted mean because the $Y_{j r}^{s}$ are no longer equal in the steady state. Again we will be concerned with $\beta$ 's that depend only on $r$, not on $i$ or $j$; so we define $\bar{\beta}_{\mu}$ :

$$
\bar{\beta}_{\mu} \equiv \sum_{r=1}^{m} \frac{k^{r-1} /(k+\mu)^{r}}{\frac{1}{\mu}\left[1-\left(\frac{k}{k+\mu}\right)^{m}\right]^{\prime}} \beta_{i j r}
$$

As $\mu \rightarrow 0, \bar{\beta}_{\mu}$ in (C8) approaches $\bar{\beta}$ for $\mu=0$. With (C8), Equation (C1) simplifies to (C9), the generalization of (18):

$$
U_{i}=c_{i} \bar{\beta}_{\mu} X_{i}^{s} \sum_{j=1}^{m} \rho_{i j} \frac{Y_{j}^{s}}{X_{j}^{s}+Y_{j}^{s}}+\mu X_{i}^{s}
$$

\section{RESTRICTED MIXING}

For restricted mixing, $\rho_{i j}$ is 0 if $i \neq j$ and 1 if $i=j$. In this case, Equation (C9) reduces to

$$
U_{i}=c_{i} X_{i}^{s} \bar{\beta}_{\mu} \frac{Y_{i}^{s}}{X_{i}^{s}+Y_{i}^{s}}+\mu X_{i}^{s}
$$


Equating $U_{i}-\mu X_{i}^{s}$ in (C4) and (C10) gives

$$
X_{i}^{s}=\frac{\mu Y_{i}^{s}}{c_{i} \bar{\beta}_{\mu}\left(1-\alpha^{m}\right)-\mu}=\frac{\left(U_{i}-\mu X_{i}\right)\left(1-\alpha^{m}\right)}{c_{i} \bar{\beta}_{\mu}\left(l-\alpha^{m}\right)-\mu} .
$$

Solving for $X_{i}^{s}$ gives

$$
X_{i}^{s}=\frac{\left(1-\alpha^{m}\right) U_{i}}{c_{i} \bar{\beta}_{\mu}\left(1-\alpha^{m}\right)-\mu \alpha^{m}}
$$

Substituting in (C4) gives $Y_{i}^{s}$ :

$$
Y_{i}^{s}=\frac{c_{i} \bar{\beta}_{\mu}\left(1-\alpha^{m}\right)-\mu}{c_{i} \bar{\beta}_{\mu}\left(1-\alpha^{m}\right)-\mu \alpha^{m}}\left(\frac{1-\alpha^{m}}{\mu}\right) U_{i} .
$$

The steady-state infected fraction then becomes

$$
\frac{Y_{i}^{s}}{X_{i}^{s}+Y_{i}^{s}}=\frac{c_{i} \bar{\beta}_{\mu}\left(1-\alpha^{m}\right)-\mu}{c_{i} \bar{\beta}_{\mu}\left(1-\alpha^{m}\right)} .
$$

For an endemic state, the numerator of ( $\mathrm{C} 14)$ [and (C13)] must be positive, giving the threshold condition for $\bar{\beta}_{\mu}$ :

$$
\bar{\beta}_{\mu}>\bar{\beta}_{\mu, T, i} \equiv \frac{\mu}{c_{i}\left(1-\alpha^{m}\right)}=\frac{1}{\frac{c_{i} m}{k}\left(\frac{k}{k+\mu}\right)^{m}\left[1+g\left(\frac{\mu}{k}\right)\right]} .
$$

Note that this reduces to the result found for $\mu=0$. For $X_{i}^{s}$ and $Y_{i}^{s}$ to remain finite, the denominators of $(\mathrm{C} 12)$ and $(\mathrm{C} 13)$ must also be positive. However, that condition is already satisfied when the numerator of (C14) is positive and so provides no additional constraint.

With the threshold condition given by $(\mathrm{C} 15)$, the endemic infected fraction is given by the same expression as Equation (21) for $\mu=0$ :

$$
\frac{Y_{i}^{s}}{X_{i}^{s}+Y_{i}^{s}}=1-\frac{\mu}{c_{i} \bar{\beta}_{\mu}\left(1-\alpha^{m}\right)}=1-\frac{\bar{\beta}_{\mu, T, i}}{\bar{\beta}_{\mu}} .
$$

PROPORTIONAL MIXING

By using the $\rho_{i j}$ for proportional mixing, Equation (C9) becomes

$$
U_{i}=c_{i} \bar{\beta}_{\mu} X_{i}^{s}\left(\frac{\sum c_{j} Y_{j}^{s}}{\sum c_{j} X_{j}^{s}+\sum c_{j} Y_{j}^{s}}\right)+\mu X_{i}^{s}
$$


Define $K_{Y}^{s}$ as in Equation (28), but for the steady state:

$$
K_{Y}^{s} \equiv \frac{\sum c_{j} Y_{j}^{s}}{\sum c_{j} X_{j}^{s}+\sum c_{j} Y_{j}^{s}}
$$

We will write $K$ for $K_{Y}^{s}$ for the rest of this discussion. Then,

$$
U_{i}=c_{i} \bar{\beta}_{\mu} X_{i}^{s} K+\mu X_{i}^{s} \quad \text { or } \quad X_{i}^{s}=\frac{U_{i}}{\left(\mu+c_{i} \bar{\beta}_{\mu} K\right)}
$$

and

$$
Y_{i}^{s}=\theta_{\mu}\left(U_{i}-\mu X_{i}^{s}\right)=\theta_{\mu}\left(U_{i}-\frac{\mu U_{i}}{\mu+c_{i} \bar{\beta}_{\mu} K}\right)=\frac{U_{i} \theta_{\mu} c_{i} \bar{\beta}_{\mu} K}{\mu+c_{i} \bar{\beta}_{\mu} K}
$$

Thus the equilibrium fraction infected in group $i$ becomes

$$
\frac{Y_{i}^{s}}{X_{i}^{s}+Y_{i}^{s}}=\frac{\theta_{\mu} c_{i} \bar{\beta}_{\mu} K}{1+\theta_{\mu} c_{i} \bar{\beta}_{\mu} K}
$$

Substitute (C19) and (C20) into (C18) and simplify:

$$
\begin{aligned}
K= & \frac{1}{\frac{\sum c_{j} X_{j}}{\sum c_{j} Y_{j}}+1}=\frac{1}{\frac{\sum c_{j} U_{j} \frac{1}{\mu+c_{j} \bar{\beta}_{\mu} K}}{K \bar{\beta}_{\mu} \theta_{\mu} \sum c_{j} U_{j} \frac{c_{j}}{\mu+c_{j} \bar{\beta}_{\mu} K}}}+1 \\
= & \frac{K}{\sum c_{j} U_{j} \frac{1}{\mu+c_{j} \bar{\beta}_{\mu} K}}+K \\
& \bar{\beta}_{\mu} \theta_{\mu} \sum c_{j} U_{j} \frac{c_{j}}{\mu+c_{j} \bar{\beta}_{\mu} K}
\end{aligned}
$$

This has a solution $K \in(0,1]$ if and only if

$$
H(K) \equiv \frac{\sum c_{j} U_{j} \frac{1}{\mu+c_{j} \bar{\beta}_{\mu} K}}{\bar{\beta}_{\mu} \theta_{\mu} \sum c_{j} U_{j} \frac{c_{j}}{\mu+c_{j} \bar{\beta}_{\mu} K}}=1-K .
$$

Clearly, $K \mapsto 1-K$ is a decreasing function of $K$. We will show that $H^{\prime}(K)>0$ and then conclude, as we did at the end of Appendix B, that 
$H(K)$ is an increasing function of $K$. Consequently, as pictured in Figure 22, their graphs can cross at at most one point and (C22) has at most one solution. By the quotient rule,

$$
\begin{aligned}
\bar{\beta}_{\mu} \theta_{\mu}\left(\sum c_{j} U_{j} \frac{c_{j}}{\mu+c_{j} \bar{\beta}_{\mu} K}\right)^{2} H^{\prime}(K) \\
=-\bar{\beta}_{\mu} \sum c_{j} U_{j} \frac{c_{j}}{\left(\mu+c_{j} \bar{\beta}_{\mu} K\right)^{2}} \sum c_{j} U_{j} \frac{c_{j}\left(\mu+c_{j} \bar{\beta}_{\mu} K\right)}{\left(\mu+c_{j} \bar{\beta}_{\mu} K\right)^{2}} \\
+\bar{\beta}_{\mu} \sum c_{j} U_{j} \frac{c_{j}^{2}}{\left(\mu+c_{j} \bar{\beta}_{\mu} K\right)^{2}} \sum c_{j} U_{j} \frac{\left(\mu+c_{j} \bar{\beta}_{\mu} K\right)}{\left(\mu+c_{j} \bar{\beta}_{\mu} K\right)^{2}} \\
=-\bar{\beta}_{\mu} \mu \sum \lambda_{j} c_{j} \sum \lambda_{j} c_{j}+\bar{\beta}_{\mu} \mu \sum \lambda_{j} c_{j}^{2} \sum \lambda_{j},
\end{aligned}
$$

which is $\geqslant 0$ by the Schwarz inequality (sce, for example, [95]). In the last line, we have used $\lambda_{j}$ for $c_{j} U_{j} /\left(\mu+c_{j} \bar{\beta}_{\mu} K\right)^{2}$.

Since $H$ is an increasing function of $K,(\mathrm{C} 22)$ has at most one solution. It will have exactly one solution if and only if the graphs of $H$ starts below the graph of $K \mapsto 1-K$, that is, if and only if $H(0)<1$, which is therefore the condition for the existence of an endemic equilibrium. It is straightforward to compute that

$$
H(0)=\frac{\sum c_{j} U_{j}}{\bar{\beta}_{\mu} \theta_{\mu} \sum c_{j}^{2} U_{j}}
$$

so the endemicity condition can be written as

$$
\frac{\sum c_{j} U_{j}}{\bar{\beta}_{\mu} \theta_{\mu} \sum c_{j}^{2} U_{j}}<1 \quad \text { or } \quad \bar{\beta}_{\mu}>\frac{\sum c_{j} U_{j}}{\theta_{\mu} \sum c_{j}^{2} U_{j}} .
$$

Note that $\Sigma c_{j} U_{j} / \Sigma U_{j}=\bar{c}_{U}$ and $\sum c_{j}^{2} U_{j} / \Sigma U_{j}=\sigma^{2}+\bar{c}_{U}^{2}$, where $\sigma^{2}$ is the variance of the $c_{j}$ for weights $U_{j}$. Thus,

$$
\frac{\sum c_{j}^{2} U_{j}}{\sum c_{j} U_{j}}=\bar{c}_{U}+\frac{\sigma^{2}}{\bar{c}_{U}} \equiv c^{*}
$$

and (C23) can be written in the form

$$
\frac{1}{\bar{\beta}_{\mu} \theta_{\mu} c^{*}}<1 \quad \text { or } \quad \bar{\beta}_{\mu}>\bar{\beta}_{\mu, T} \equiv \frac{1}{\theta_{\mu} c^{*}},
$$


giving a threshold $\bar{\beta}_{\mu, T}$ in $\bar{\beta}_{\mu}$. This has the same form as Equation (32) but with $\theta_{\mu}$ replacing $\mathrm{m} / k$ and $c^{*}$ replacing $\bar{c}_{U}$. Anderson et al. [68] used a similar quadratic average, but they used the total number in each group as the weight, not the recruitment rate; but see under Proportional Mixing Section 9 and Equation (53).

It is of interest to carry out the steady-state calculations in a pattern following that used for $\mu=0$. Define $\bar{c}_{Y}$ as in Equation (24). Then at the steady state $\bar{c}_{Y}$ becomes $\bar{c}_{w}$, as defined in the equation

$$
\bar{c}_{Y}^{s}=\bar{c}_{w}=\frac{\sum c_{j} W_{j}}{\sum W_{j}} .
$$

Sum (C17) to obtain

$$
\sum W_{j}=\bar{\beta}_{\mu} \frac{\sum c_{j} X_{j}^{s} \sum c_{j} Y_{j}^{s}}{\sum c_{j} X_{j}^{s}+\sum c_{j} Y_{j}^{s}}
$$

Solve (C27) for $\sum c_{j} X_{j}^{s}$ :

$$
\sum c_{j} X_{j}^{s}=\frac{\bar{c}_{w} \theta_{\mu} \Sigma W_{j}}{\bar{\beta}_{\mu} \bar{c}_{w} \theta_{\mu}-1}
$$

Plug (C23) and (C28) into the definition of (C18) of $K$ to obtain

$$
K=K_{Y}^{s}=\frac{\bar{\beta}_{\mu} \bar{c}_{w} \theta_{\mu}-1}{\bar{\beta}_{\mu} \bar{c}_{w} \theta_{\mu}} .
$$

Substitute (C29) in (C19) and (C20) and solve for $X_{i}^{s}$ and $Y_{i}^{s}$ :

$$
\begin{aligned}
X_{i}^{s} & =\frac{U_{i} \theta_{\mu}}{\mu \theta_{\mu}+\frac{c_{i}}{\bar{c}_{w}}\left(\bar{\beta}_{\mu} \bar{c}_{w} \theta_{\mu}-1\right)} \\
Y_{i}^{s} & =\frac{\theta_{\mu} U_{i}\left(c_{i} / \bar{c}_{w}\right)\left(\bar{\beta}_{\mu} \bar{c}_{w} \theta_{\mu}-1\right)}{\mu \theta_{\mu}+\left(c_{i} / \bar{c}_{w}\right)\left(\bar{\beta}_{\mu} \bar{c}_{w} \theta_{\mu}-1\right)} .
\end{aligned}
$$

In order for an endemic cquilibrium to exist, the numcrator of $Y_{i}^{s}$ in (C31) must be positive, giving an endemicity threshold

$$
\bar{\beta}_{\mu} \bar{c}_{w} \theta_{\mu}>1 \quad \text { or } \quad \bar{\beta}_{\mu}>\bar{\beta}_{\mu, T, i} \equiv \frac{1}{\bar{c}_{w} \theta_{\mu}},
$$

which is analogous in form to Equations (32) and (C25). Finally, the infected 
fraction in group $i$ becomes

$$
\frac{Y_{i}^{s}}{X_{i}^{s}+Y_{i}^{s}}=\frac{\left(c_{i} / \bar{c}_{w}\right)\left[\bar{\beta}_{\mu} / \bar{\beta}_{\mu, T, i}-1\right]}{1+\left(c_{i} / \bar{c}_{w}\right)\left[\bar{\beta}_{\mu} / \bar{\beta}_{\mu, T, i}-1\right]}
$$

These last five expressions should be compared with the corresponding equations in Section 7.

PREFERRED MIXING

For preferred mixing, Equation (C9) becomes

$$
U_{i}-\mu X_{i}^{s}=\bar{\beta}_{\mu} c_{i} X_{i}^{s}\left[\frac{\rho_{i} Y_{i}^{s}}{X_{i}^{s}+Y_{i}^{s}}+\frac{\left(1-\rho_{i}\right) \sum c_{j}\left(1-\rho_{j}\right) Y_{j}^{s}}{\sum c_{j}\left(1-\rho_{j}\right) X_{j}^{s}+\sum c_{j}\left(1-\rho_{j}\right) Y_{j}^{s}}\right]
$$

Using $W_{i}=U_{i}-\mu X_{i}^{s}$ and $K$ and $K^{s}$ as in Equation (34), (C34) becomes

$$
W_{i}=\bar{\beta}_{\mu} c_{i} X_{i}^{s}\left[\frac{\rho_{i} Y_{i}^{s}}{X_{i}^{s}+Y_{i}^{s}}+\left(1-\rho_{i}\right) K_{Y}^{s}\right] \text {. }
$$

Multiplying this expression out and using $Y_{i}^{s}=\theta_{\mu} W_{i}$, one obtains a quadratic equation in $X_{i}^{s}$, similar to Equation (35):

$\bar{\beta}_{\mu} c_{i}\left(1-\rho_{i}\right) K\left(X_{i}^{s}\right)^{2}+\left[\bar{\beta}_{\mu} c_{i} \theta_{\mu}\left(\left(1-\rho_{i}\right) K+\rho_{i}\right)-1\right] W_{i} X_{i}^{s}-\theta_{\mu} W_{i}^{2}=0$,

where we write $K$ for $K_{V}^{s}$ for the rest of this appendix. We seek a solution of (C35) that is the analogue of (36):

$$
X_{i}^{s}=\frac{W_{i}\left(-P_{i}+\sqrt{P_{i}^{2}+Q_{i}}\right)}{2 \bar{\beta}_{\mu} c_{i}\left(1-\rho_{i}\right) K}
$$

where

$$
P_{i}=\bar{\beta}_{\mu} c_{i} \theta_{\mu}\left(\left(1-\rho_{i}\right) K+\rho_{i}\right)-1 \quad \text { and } \quad Q_{i}=4 \bar{\beta}_{\mu} c_{i} \theta_{\mu}\left(1-\rho_{i}\right) K
$$

Let

$$
A_{i} \equiv A_{i}(K)=\frac{-P_{i}+\sqrt{P_{i}^{2}+Q_{i}}}{2 c_{i} \bar{\beta}_{\mu}\left(1-\rho_{i}\right)}
$$

so that, dropping the $s$ superscript,

$$
X_{i}=W_{i} A_{i} / K
$$


Plug in $W_{i}=U_{i}-\mu X_{i}$ into (C37) and solve for $X_{i}$ to find

$$
X_{i}=U_{i} A_{i} /\left(K+\mu A_{i}\right)
$$

On the other hand,

$$
W_{i}=U_{i}-\mu X_{i}=U_{i}\left(1-\frac{\mu A_{i}}{K+\mu A_{i}}\right)=\frac{U_{i} K}{K+\mu A_{i}}
$$

and

$$
Y_{i}=\theta_{\mu} W_{i}=\frac{\theta_{\mu} U_{i} K}{K+\mu A_{i}}
$$

Substitute (C38) and (C39) into the formula for $K_{Y}$ to obtain $T(K)$ :

$$
\begin{aligned}
T(K) & \equiv \frac{\sum c_{i}\left(1-\rho_{i}\right) Y_{i}}{\sum c_{i}\left(1-\rho_{i}\right) X_{i}+\sum c_{i}\left(1-\rho_{i}\right) Y_{i}} \\
& =\frac{1}{\frac{\sum c_{i}\left(1-\rho_{i}\right) X_{i}}{\sum c_{i}\left(1-\rho_{i}\right) Y_{i}}+1} \\
& =\frac{1}{\frac{\sum c_{i}\left(1-\rho_{i}\right) U_{i} \frac{A_{i}}{K+\mu A_{i}}}{\sum c_{i}\left(1-\rho_{i}\right) U_{i} \theta_{\mu} \frac{K}{K+\mu A_{i}}}+1} \\
& =\frac{K}{\sum c_{i}\left(1-\rho_{i}\right) U_{i} \frac{A_{i}}{K+\mu A_{i}}}+K \\
& \theta_{\mu} \sum c_{i}\left(1-\rho_{i}\right) U_{i} \frac{1}{K+\mu A_{i}}
\end{aligned} .
$$

We are looking for a nonzero solution $K$ of $T(K)=K$, that is, a solution of

$$
H(K) \equiv \frac{\sum c_{i}\left(1-\rho_{i}\right) U_{i} \frac{A_{i}}{K+\mu A_{i}}}{\theta_{\mu} \sum c_{i}\left(1-\rho_{i}\right) U_{i} \frac{1}{K+\mu A_{i}}}=1-K
$$

As illustrated in Figure 22, the graph of $K \rightarrow 1-K$ is a negatively sloped straight line. As we did in Appendix B and in the calculations for proportional mixing, we will show that the graph of $H(K)$ slopes upward, i.e., that 
$H^{\prime}(K)>0$. Write $\bar{\lambda}_{i}$ for $c_{i}\left(1-\rho_{i}\right) U_{i}$. By the quotient rule,

$$
\begin{aligned}
\left(\sum \bar{\lambda}_{i} \frac{1}{K+\mu A_{i}}\right)^{2} \theta_{\mu} H^{\prime}(K)= & \sum \bar{\lambda}_{i} \frac{\left(A_{i}^{\prime} K-A_{i}\right)}{\left(K+\mu A_{i}\right)^{2}} \sum \bar{\lambda}_{i} \frac{1}{K+\mu A_{i}} \\
& +\sum \bar{\lambda}_{i} \frac{A_{i}}{K+\mu A_{i}} \sum \bar{\lambda}_{i} \frac{\left(1+\mu A_{i}^{\prime}\right)}{\left(K+\mu A_{i}\right)^{2}} \\
\geqslant & -\sum \bar{\lambda}_{i} \frac{A_{i}}{\left(K+\mu A_{i}\right)^{2}} \sum \bar{\lambda}_{i} \frac{1}{K+\mu A_{i}} \\
& +\sum \bar{\lambda}_{i} \frac{A_{i}}{K+\mu A_{i}} \sum \bar{\lambda}_{i} \overline{\left(K+\mu A_{i}\right)^{2}} \\
& \left(\operatorname{since} \frac{1}{\left.A^{\prime} \geqslant 0 \text { by the argument in Appendix B }\right)}\right. \\
= & -\sum \bar{\lambda}_{i} \frac{A_{i}}{\left(K+\mu A_{i}\right)^{2}} \sum \bar{\lambda}_{i} \frac{K+\mu A_{i}}{\left(K+\mu A_{i}\right)^{2}} \\
& +\sum \bar{\lambda}_{i} \frac{\left(A_{i} K+\mu A_{i}^{2}\right)}{\left(K+\mu A_{i}\right)^{2}} \sum \bar{\lambda}_{i} \frac{1}{\left(K+\mu A_{i}\right)^{2}} \\
= & -\mu \sum \bar{\lambda}_{i} \frac{A_{i}}{\left(K+\mu A_{i}\right)^{2}} \sum \bar{\lambda}_{i} \frac{A_{i}}{\left(K+\mu A_{i}\right)^{2}} \\
& +\mu \sum \bar{\lambda}_{i} \frac{1}{\left(K+\mu A_{i}\right)^{2}} \sum \bar{\lambda}_{i} \frac{1}{\left(K+\mu A_{i}\right)^{2}} \\
= &
\end{aligned}
$$

by the Schwarz inequality again.

Since $H^{\prime}>0$, the graph of $H$ curves upwards as in Figure 22. Therefore, the graph of $H$ and the graph of $K \mapsto 1-K$ can cross at most at one point. Furthermore, they cross if and only if the graph of $H$ starts below 1, i.e., if and only if $H(0)<1$. As in Appendix $\mathrm{B}$, the threshold condition is given by $H(0)$ :

$$
\begin{aligned}
H(0) & =\frac{\sum c_{i}\left(1-\rho_{i}\right) U_{i} \frac{A_{i}(0)}{0+\mu A_{i}(0)}}{\theta_{\mu} \sum c_{i}\left(1-\rho_{i}\right) U_{i} \frac{1}{0+\mu A_{i}(0)}}=\frac{\sum c_{i}\left(1-\rho_{i}\right) U_{i}}{\theta_{\mu} \sum c_{i}\left(1-\rho_{i}\right) U_{i} \frac{1}{A_{i}(0)}} \\
& =\frac{\sum c_{i}\left(1-\rho_{i}\right) U_{i}}{\theta_{\mu} \bar{\beta}_{\mu} \sum c_{i}^{2}\left(1-\rho_{i}\right)^{2} U_{i} \frac{1}{\max \left\{0,1-c_{i} \bar{\beta}_{\mu} \theta_{\mu} \rho_{i}\right\}}} .
\end{aligned}
$$

Note that for proportional mixing, $\rho_{i}=0$ for all $i$ and (C40) reduces to (C23), the condition derived for proportional mixing. 


\section{APPENDIX D. DEPENDENCE OF THE EQUILIBRIA AND THRESHOLDS ON PARAMETERS}

In this appendix, we calculate the effect of varying the parameters of the model (1)-(4) on the equilibria $X_{i}^{s}$ and $Y_{i}^{s} /\left(X_{i}^{s}+Y_{i}^{s}\right)$ for preferred mixing. Since the equilibrium equations (33) for the $X_{i}^{s}$ are implicit equations, the equations for the partial derivatives of the $X_{i}^{s}$ are a system of linear equations $A \mathbf{x}=\mathbf{b}$, where $x_{i}$ is the derivative of $X_{i}^{s}$ with respect to some parameter. The matrices $A=\left(\left(a_{i j}\right)\right)$ that arise in these calculations are all dominant diagonal matrices with positive diagonals and nonpositive offdiagonals:

$$
a_{i i}>0, a_{i j}<0 \quad(i \neq j) \quad \text { and } \quad \sum_{h} a_{h j}>0 \quad \text { for all } i, j .
$$

Such matrices arise frequently in economic analysis (see [96]) and in biological models (see [76]). In economic models, however, we usually have $b_{j}>0$ for all $j$ and can conclude that each component $x_{i}$ of the solution $\mathbf{x}$ is positive. In our systems, the $b_{j}$ have different signs. The following theorem provides the information we need to keep track of the signs of the $x_{i}$. See [97] for its proof.

THEOREM D. 1

Let $\mathbf{b}$ be a fixed n-vector. Suppose that $b_{j}>0$ and that

$$
b_{j}+\sum_{i \in S} b_{i}>0, \quad \text { where } S \equiv\left\{i=1, \ldots, n: b_{i}<0\right\} .
$$

(1) Then, for any matrix $A$ satisfying (D1), the $j$ th component $x_{j}$ of the solution of $A \mathrm{x}=\mathrm{b}$ is positive.

(2) If (D2) is violated, then there exist matrices $A_{1}$ and $A_{2}$ both satisfying (D1) such that the $j$ th component $x_{j}$ of the solution of $A_{1} \mathbf{x}=\mathbf{b}$ is positive and the $j$ th component $x_{j}$ of the solution of $A_{2} \mathbf{x}=\mathbf{b}$ is negative.

Remark 1. Condition (D2) is easily seen to be equivalent to

$$
b_{j}+\sum_{i \in T} b_{i}>0 \quad \text { for all subsets } T \text { of }\{1, \ldots, j-1, j+1, \ldots, n\} .
$$

This is the condition we will often use in applying this theorem.

Remark 2. If $\mathbf{x}^{*}$ is the solution of $A \mathbf{x}=\mathbf{b}$, then $-\mathbf{x}^{*}$ is the solution of $A \mathbf{x}=-\mathbf{b}$. It follows that if, in the above notation, $b_{j}<0$ and $b_{j}+\sum_{i}\left\{b_{i}\right.$ : $\left.b_{i}>0\right\}<0$, then $x_{j}<0$.

Remark 3. Throughout this appendix, we will omit the superscript $s$ on the steady-state $X_{i}^{s}, Y_{i}^{s}$, and $K^{s}$ for ease of notation. 
HIV TRANSMISSION: EFFECT OF CONTACT PATTERNS

DEPENDENCE ON $c_{i}$

\section{THEOREM D.2}

For preferred mixing with $\mu=0$,

$$
\frac{\partial X_{i}}{\partial c_{i}}<0 \quad \text { and } \quad \frac{\partial}{\partial c_{i}}\left(\frac{Y_{i}}{X_{i}+Y_{i}}\right)>0
$$

for all $i$. If $Y_{i} /\left(X_{i}+Y_{i}\right)<K_{Y}$, then

$$
\frac{\partial X_{j}}{\partial c_{i}}<0 \quad \text { and } \quad \frac{\partial}{\partial c_{i}}\left(\frac{Y_{j}}{X_{j}+Y_{j}}\right)>0
$$

for all $i$ and $j$.

Proof. Without loss of generality, we will work with $i=1$; the same proof carries over for general $i$. Differentiate with respect to $c_{1}$ each of the following $n$ equations:

$$
U_{i}=\bar{\beta} c_{i} X_{i}\left(\rho_{i} \frac{Y_{i}}{X_{i}+Y_{i}}+\left(1-\rho_{i}\right) \frac{\sum_{j} c_{j}\left(1-\rho_{j}\right) Y_{j}}{\sum_{j} c_{j}\left(1-\rho_{j}\right) X_{j}+\sum_{j} c_{j}\left(1-\rho_{j}\right) Y_{j}}\right)
$$

for $i=1,2, \ldots, n$. The result is the following systems of linear equations in the $n$ variables $\partial X_{1} / \partial c_{1}, \ldots, \partial X_{n} / \partial c_{1}$ :

$$
\left(\begin{array}{ccc}
a_{11} & \cdots & a_{1 n} \\
\vdots & \ddots & \vdots \\
a_{n 1} & \cdots & a_{n n}
\end{array}\right)\left(\begin{array}{c}
\partial X_{1} / \partial c_{1} \\
\vdots \\
\partial X_{n} / \partial c_{1}
\end{array}\right)=\left(\begin{array}{c}
b_{1} \\
\vdots \\
b_{n}
\end{array}\right)
$$

where

$$
\begin{aligned}
a_{j j}= & \frac{\rho_{j} c_{j} Y_{j}^{2}}{\left(X_{y}+Y_{j}\right)^{2}}+c_{j}\left(1-\rho_{j}\right) K^{2}+\frac{c_{j}\left(1-\rho_{j}\right) K \sum_{h \neq j} c_{h}\left(1-\rho_{h}\right) X_{h}}{\left[\sum_{h} c_{h}\left(1-\rho_{h}\right) X_{h}+\sum_{h} c_{h}\left(1-\rho_{h}\right) Y_{h}\right]}>0 \\
a_{i j}= & \frac{-c_{j}\left(1-\rho_{j}\right) K c_{i}\left(1-\rho_{i}\right) X_{i}}{\left[\sum_{h} c_{h}\left(1-\rho_{h}\right) X_{h}+\sum_{h} c_{h}\left(1-\rho_{h}\right) Y_{h}\right]}<0, \quad i \neq j \\
h_{1}= & -X_{1}\left[\frac{\rho_{1} Y_{1}}{X_{1}+Y_{1}}+\frac{\left(1-\rho_{1}\right) K \sum_{h \neq 1} c_{h}\left(1-\rho_{h}\right) X_{h}}{\left[\sum_{h} c_{h}\left(1-\rho_{h}\right) X_{h}+\sum_{h} c_{h}\left(1-\rho_{h}\right) Y_{h}\right]}+\left(1-\rho_{1}\right) K^{2}\right] \\
& -X_{1}\left(1-\rho_{1}\right) \frac{c_{1}\left(1-\rho_{1}\right) Y_{1}}{\left[\sum_{h} c_{h}\left(1-\rho_{h}\right) X_{h}+\Sigma_{h} c_{h}\left(1-\rho_{h}\right) Y_{h}\right]}(1-K)<0 \\
b_{j}= & -X_{j}\left(1-\rho_{j}\right) c_{j}\left[\frac{\left(1-\rho_{1}\right) Y_{1}(1-K)-\left(1-\rho_{1}\right) X_{1} K}{\left[\sum_{h} c_{h}\left(1-\rho_{h}\right) X_{h}+\sum_{h} c_{h}\left(1-\rho_{h}\right) Y_{h}\right]}\right], \quad j=2, \ldots, n .
\end{aligned}
$$


One computes that for each column $j$ of the matrix $A=\left(\left(a_{i j}\right)\right)$,

$$
\sum_{i=1}^{n} a_{i j}=\frac{\rho_{j} c_{j} Y_{j}^{2}}{\left(X_{j}+Y_{j}\right)^{2}}+c_{j}\left(1-\rho_{j}\right) K^{2}>0
$$

and for the right-hand side $b$,

$$
\begin{aligned}
\sum_{i=1}^{r} b_{i}= & -\left[\frac{\rho_{1} X_{1} Y_{1}}{X_{1}+Y_{1}}+\left(1-\rho_{1}\right)\left(X_{1} K^{2}+Y_{1}(1-K)^{2}\right)\right] \\
& -\frac{\left(1-\rho_{1}\right) X_{1} \sum_{h=r+1}^{n} c_{h}\left(1-\rho_{h}\right) X_{h}}{\sum_{h} c_{h}\left(1-\rho_{h}\right) X_{h}+\sum_{h} c_{h}\left(1-\rho_{h}\right) Y_{h}}<0 .
\end{aligned}
$$

Since $A$ satisfies (D1), $b_{1}<0$, and $\Sigma_{1, J} b_{i}<0$ for any subset $J$ of $\{2, \ldots, n\}$, $\partial X_{1} / \partial c_{1}<0$. Since $\partial Y_{1} / \partial c_{1}=0$,

$$
\frac{\partial}{\partial c_{1}}\left(\frac{Y_{1}}{X_{1}+Y_{1}}\right)>0
$$

the infected fraction in group 1 increases as sexual activity in group 1 increases. Furthermore, if

$$
Y_{1}(1-K)-X_{1} K>0
$$

then all the $b_{j}$ are negative, and for every $i$,

$$
\frac{\partial X_{i}}{\partial c_{1}}<0 \quad \text { and } \quad \frac{\partial}{\partial c_{1}}\left(\frac{Y_{i}}{X_{i}+\bar{Y}_{i}}\right)>0
$$

Condition (D5) is equivalent to

$$
\frac{Y_{1}}{X_{1}+Y_{1}}<K_{Y}
$$

DEPENDENCE ON $\bar{\beta}$

THEOREM D.3

For preferred mixing and $\mu=0$,

$$
\frac{\partial X_{i}}{\partial \bar{\beta}}<0 \quad \text { and } \quad \frac{\partial}{\partial \bar{\beta}}\left(\frac{Y_{i}}{X_{i}+Y_{i}}\right)>0
$$

for all $i$. 
Proof. Once again differentiate the $n$ equations (D4), this time with respect to $\bar{\beta}$. The result is a linear system:

$$
\left(\begin{array}{ccc}
a_{11} & \cdots & a_{1 n} \\
\vdots & \ddots & \vdots \\
a_{n 1} & \cdots & a_{n n}
\end{array}\right)\left(\begin{array}{c}
\partial X_{1} / \partial \bar{\beta} \\
\vdots \\
\partial X_{n} / \partial \bar{\beta}
\end{array}\right)=\left(\begin{array}{c}
b_{1} \\
\vdots \\
b_{n}
\end{array}\right),
$$

where

$$
\begin{aligned}
a_{j j} & =\frac{\bar{\beta} \rho_{j} c_{j} Y_{j}^{2}}{\left(X_{y}+Y_{j}\right)^{2}}+\bar{\beta} c_{j}\left(1-\rho_{j}\right) K-\frac{\bar{\beta} c_{j}\left(1-\rho_{j}\right) K X_{j} c_{j}\left(1-\rho_{j}\right)}{\left[\sum_{h} c_{h}\left(1-\rho_{h}\right) X_{h}+\sum_{h} c_{h}\left(1-\rho_{h}\right) Y_{h}\right]}>0 \\
a_{i j} & =\frac{-\bar{\beta} c_{j}\left(1-\rho_{j}\right) K c_{i}\left(1-\rho_{i}\right) X_{i}}{\left[\sum_{h} c_{h}\left(1-\rho_{h}\right) X_{h}+\sum_{h} c_{h}\left(1-\rho_{h}\right) Y_{h}\right]}<0, \quad i \neq j \\
b_{j} & =-X_{j} c_{j}\left[\rho_{j} \frac{Y_{j}}{X_{j}+Y_{j}}+\left(1-\rho_{j}\right) K\right]<0 \quad \text { for all } j .
\end{aligned}
$$

Once again the matrix $A$ is a dominant diagonal matrix. This time the right-hand-side vector $\mathbf{b}$ has every component negative. By Theorem D.1, each solution $\partial X_{i} / \partial \bar{\beta}$ is negative. Since $\partial Y_{i} / \partial \bar{\beta}=0$,

$$
\frac{\partial}{\partial \bar{\beta}}\left(\frac{Y_{i}}{X_{i}+Y_{i}}\right)>0
$$

Since $\bar{\beta}$ is a weighted average of the $\beta_{i j k}$, it follows immediately that if any of the $\beta_{i j k}$ rises, each $X_{h}$ decreases and each equilibrium fraction infected increases.

DEPENDENCE ON $\rho_{i}$

THEOREM D.4

For preferred mixing with $\mu=0$, if $Y_{i} /\left(X_{i}+Y_{i}\right)<K_{Y}$, then

$$
\frac{\partial X_{i}}{\partial \rho_{i}}>0 \quad \text { and } \quad \frac{\partial}{\partial \rho_{i}}\left(\frac{Y_{i}}{X_{i}+Y_{i}}\right)<0 .
$$

In this case,

$$
\frac{\partial}{\partial \rho_{i}}\left(\frac{Y_{j}}{X_{j}+Y_{j}}\right)
$$

is indeterminate. On the other hand, if $Y_{i} /\left(X_{i}+Y_{i}\right)>K_{Y}$, and if $c_{i}\left(1-\rho_{i}\right) Y_{i}$ 
is large compared with $c_{h}\left(1-\rho_{h}\right) X_{h}$ for $h \neq i, j$, then

$$
\frac{\partial X_{j}}{\partial \rho_{i}}>0 \quad \text { and } \quad \frac{\partial}{\partial \rho_{i}}\left(\frac{Y_{j}}{X_{j}+Y_{j}}\right)<0
$$

Proof. For ease of notation and calculation we will work with $\sigma_{i} \equiv 1-\rho_{i}$ for $i=1, \ldots, n$. Then (D4) becomes

$$
U_{i}=\bar{\beta} c_{i} X_{i}\left(\left(1-\sigma_{i}\right) \frac{Y_{i}}{X_{i}+Y_{i}}+\sigma_{i} \frac{\sum_{j} c_{j} \sigma_{y} Y_{j}}{\sum_{j} c_{j} \sigma_{j} X_{j}+\sum_{j} c_{j} \sigma_{j} Y_{j}}\right)
$$

for $i=1, \ldots, n$. As usual, we will work without loss of generality with $\sigma_{1}$. Taking the derivative with respect to $\sigma_{1}$ of each of the $n$ equations in (D6) leads to the linear system

$$
\left(\begin{array}{ccc}
a_{11} & \cdots & a_{1 n} \\
\vdots & \ddots & \vdots \\
a_{n 1} & \cdots & a_{n n}
\end{array}\right)\left(\begin{array}{c}
\partial X_{1} / \partial \sigma_{1} \\
\vdots \\
\partial X_{n} / \partial \sigma_{1}
\end{array}\right)=\left(\begin{array}{c}
b_{1} \\
\vdots \\
b_{n}
\end{array}\right)
$$

where

$$
\begin{aligned}
a_{j j} & =c_{j}\left(1-\sigma_{j}\right)\left(\frac{Y_{j}}{X_{j}+Y_{j}}\right)^{2}+c_{j} \sigma_{j} K-\frac{c_{j} \sigma_{j} X_{j} c_{j} \sigma_{j} K}{\sum_{h} c_{h} \sigma_{h} X_{h}+\sum_{h} c_{h} \sigma_{h} Y_{h}} \\
a_{i j} & =-\frac{c_{i} \sigma_{i} X_{i} c_{j} \sigma_{j} K}{\sum_{h} c_{h} \sigma_{h} X_{h}+\sum_{h} c_{h} \sigma_{h} Y_{h}} \quad \text { for } i \neq j \\
b_{1} & =-c_{1} X_{1}\left[-\frac{Y_{1}}{X_{1}+Y_{1}}+K+\frac{\sigma_{1} c_{1} Y_{1}\left(\sum_{h} c_{h} \sigma_{h} X_{h}\right)-\sigma_{1} c_{1} X_{1}\left(\sum_{h} c_{h} \sigma_{h} Y_{h}\right)}{\left(\sum_{h} c_{h} \sigma_{h} X_{h}+\sum_{h} c_{h} \sigma_{h} Y_{h}\right)^{2}}\right] \\
& =-c_{1} X_{1}\left(K-\frac{Y_{1}}{X_{1}+Y_{1}}\right) \frac{\sum_{h \neq 1}\left(c_{h} \sigma_{h} X_{h}+c_{h} \sigma_{h} Y_{h}\right)}{\sum_{h}\left(c_{h} \sigma_{h} X_{h}+c_{h} \eta_{h} Y_{h}\right)} \\
b_{i} & =-\frac{c_{1} Y_{1} c_{i} \sigma_{i} X_{i}\left(\sum_{h} c_{h} \sigma_{h} X_{h}\right)-c_{1} X_{1} c_{i} \sigma_{i} X_{i}\left(\sum_{h} c_{h} \sigma_{h} Y_{h}\right)}{\left(\sum_{h} c_{h} \sigma_{h} X_{h}+\sum_{h} c_{h} \sigma_{h} Y_{h}\right)^{2}} . \\
& =c_{1}\left(X_{1}+Y_{1}\right) \frac{c_{i} \sigma_{i} X_{i}}{\sum_{h}\left(c_{h} \sigma_{h} X_{h}+c_{h} \sigma_{h} Y_{h}\right)}\left(K-\frac{Y_{1}}{X_{1}+Y_{1}}\right) .
\end{aligned}
$$

Once again the matrix $A$ is a dominant diagonal matrix since each off-diago- 
nal entry is clearly negative, but each column sum is

$$
\begin{aligned}
\sum_{i} a_{i j} & =c_{j}\left(1-\sigma_{j}\right) \frac{Y_{j}^{2}}{\left(X_{j}+Y_{j}\right)^{2}}+c_{j} \sigma_{j} K-c_{j} \sigma_{j} K \frac{\sum_{i} c_{i} \sigma_{i} X_{i}}{\sum_{h} c_{h} \sigma_{h} X_{h}+\sum_{h} c_{h} \sigma_{h} Y_{h}} \\
& =c_{j}\left(1-\sigma_{j}\right) \frac{Y_{j}^{2}}{\left(X_{j}+Y_{j}\right)^{2}}+c_{j} \sigma_{j} K-c_{j} \sigma_{j} K(1-K) \\
& =c_{j}\left(1-\sigma_{j}\right) \frac{Y_{j}^{2}}{\left(X_{j}+Y_{j}\right)^{2}}+c_{j} \sigma_{j} K^{2} \\
& >0
\end{aligned}
$$

Furthermore,

$$
\sum_{h} b_{h}=-c_{1}\left(X_{1}+Y_{1}\right)\left(K-\frac{Y_{1}}{X_{1}+Y_{1}}\right)^{2}<0 .
$$

If $Y_{1} /\left(X_{1}+Y_{1}\right)<K$, then

$$
b_{1}<0, \quad b_{i}>0(i \neq 1), \quad \text { and } \quad \sum_{h} b_{h}<0 .
$$

By Theorem D.1, $\partial X_{1} / \partial \sigma_{1}<0$. By the definition of $\sigma_{1}$,

$$
\frac{\partial X_{1}}{\partial \rho_{1}}>0
$$

Since $\partial Y_{i} / \partial \rho_{j}=0$ for all $i, j$,

$$
\frac{\partial}{\partial \rho_{1}}\left(\frac{Y_{1}}{X_{1}+Y_{1}}\right)<0 .
$$

If $Y_{1} /\left(X_{1}+Y_{1}\right)>K$, then $b_{1}>0$ and $b_{i}<0$ for all $i \neq 1$. In this case, $b_{1}+b_{2}<0$ if and only if

$$
\frac{c_{2} \sigma_{2} X_{2}}{\sum_{h \neq 1}\left(c_{h} \sigma_{h} X_{h}+c_{h} \sigma_{h} Y_{h}\right)}>\frac{X_{1}}{X_{1}+Y_{1}}
$$

if and only if

$$
\frac{c_{1} \sigma_{1} X_{1}+c_{2} \sigma_{2} X_{2}}{\sum_{h}\left(c_{h} \sigma_{h} X_{h}+c_{h} \sigma_{h} Y_{h}\right)}>\frac{X_{1}}{X_{1}+Y_{1}}
$$


Note that (D7) implies that

$$
1-K>\frac{X_{1}}{X_{1}+Y_{1}} \quad \text { or } \quad \frac{Y_{1}}{X_{1}+Y_{1}}>K .
$$

Therefore, if (D7) holds, then by Theorem D.1, $\partial X_{2} / \partial \sigma_{1}<0$, which in turn implies

$$
\frac{\partial X_{2}}{\partial \rho_{1}}>0 \quad \text { and } \quad \frac{\partial}{\partial \rho_{1}}\left(\frac{Y_{2}}{X_{2}+Y_{2}}\right)<0 .
$$

DEPENDENCE ON $U_{i}$

\section{THEOREM D.5}

For preferred mixing with $\mu=0$, let

$$
\omega \equiv 1-\frac{m}{k} c_{1} \bar{\beta}\left(\rho_{1} \frac{X_{1}^{2}}{\left(X_{1}+Y_{1}\right)^{2}}+\left(1-\rho_{1}\right)(1-K)^{2}\right) .
$$

If $\omega>0$, then

$$
\frac{\partial X_{1}}{\partial U_{1}}>0
$$

Two conditions that imply that $\omega>0$ are

$$
\frac{Y_{1}}{X_{1}+Y_{1}}<K \quad \text { and } \quad c_{1} \bar{\beta} \frac{m}{k}<1 .
$$

Proof. A usual, we work with $i=1$. Once again, differentiate the $n$ equations of (D4), this time with respect to $U_{1}$. The resulting linear equation is

where

$$
\left(\begin{array}{ccc}
a_{11} & \cdots & a_{1 n} \\
\vdots & \ddots & \vdots \\
a_{n 1} & \cdots & a_{n n}
\end{array}\right)\left(\begin{array}{c}
\partial X_{1} / \partial U_{1} \\
\vdots \\
\partial X_{n} / \partial U_{1}
\end{array}\right)=\left(\begin{array}{c}
b_{1} \\
\vdots \\
b_{n}
\end{array}\right)
$$

$$
\begin{aligned}
a_{j j} & =\frac{c_{j} \rho_{j} \bar{\beta} Y_{j}^{2}}{\left(X_{j}+Y_{j}\right)^{2}}+\bar{\beta} c_{j}\left(1-\rho_{j}\right) K \frac{\sum_{h \neq j} c_{h}\left(1-\rho_{h}\right) X_{h}+\sum_{h} c_{h}\left(1-\rho_{h}\right) Y_{h}}{\sum_{h} c_{h}\left(1-\rho_{h}\right) X_{h}+\sum_{h} c_{h}\left(1-\rho_{h}\right) Y_{h}}>0 \\
a_{i j} & =-\frac{c_{i}\left(1-\rho_{i}\right) X_{i} K \bar{\beta} c_{j}\left(1-\rho_{j}\right)}{\sum_{h} c_{h}\left(1-\rho_{h}\right) X_{h}+\sum_{h} c_{h}\left(1-\rho_{h}\right) Y_{h}}<0, \quad i \neq j \\
b_{1} & =1-c_{1} \frac{m}{k} \bar{\beta} \rho_{1} \frac{X_{1}^{2}}{\left(X_{1}+Y_{1}\right)^{2}}-\left(1-\rho_{1}\right) \frac{c_{1} \bar{\beta}(m / k)(1-K) c_{1}\left(1-\rho_{1}\right) X_{1}}{\sum_{h} c_{h}\left(1-\rho_{h}\right) X_{h}+\sum_{h} c_{h}\left(1-\rho_{h}\right) Y_{h}} \\
b_{j} & =-\left(1-\rho_{1}\right) \frac{c_{1} \bar{\beta}(m / k)(1-K) c_{j}\left(1-\rho_{j}\right) X_{j}}{\sum_{h} c_{h}\left(1-\rho_{h}\right) X_{h}+\sum_{h} c_{h}\left(1-\rho_{h}\right) Y_{h}}<0, \quad j \neq 1 .
\end{aligned}
$$


Once again, the matrix $A$ is a dominant diagonal matrix because

$$
\sum_{i=1}^{n} a_{i j}=c_{j} \rho_{j}\left(\frac{Y_{j}}{X_{j}+Y_{j}}\right)^{2}+c_{j}\left(1-\rho_{j}\right) \bar{\beta} K^{2}>0
$$

In addition,

$$
\sum_{j=1}^{n} b_{j}=1-\frac{m}{k} c_{1} \bar{\beta}\left(\rho_{1} \frac{X_{1}^{2}}{\left(X_{1}+Y_{1}\right)^{2}}+\left(1-\rho_{1}\right)(1-K)^{2}\right) .
$$

We first show that $b_{1}$ is positive. Equations (D4) and (16) imply that

$$
1=\frac{m}{k} c_{1} \bar{\beta}\left[\rho_{1} \frac{X_{1}}{X_{1}+Y_{1}}+\left(1-\rho_{1}\right) \frac{K X_{1}}{Y_{1}}\right] \text {. }
$$

Substitute (D9) into the expression for $b_{1}$ to obtain

$$
\begin{aligned}
b_{1}=\frac{m}{k} c_{1} \bar{\beta}\left\{\rho_{1}\right. & \frac{X_{1} Y_{1}}{\left(X_{1}+Y_{1}\right)^{2}}+\left(1-\rho_{1}\right) \\
& \left.\quad \times\left[\frac{K X_{1}}{Y_{1}}-\frac{(1-K) c_{1}\left(1-\rho_{1}\right) X_{1}}{\sum_{h} c_{h}\left(1-\rho_{h}\right) X_{h}+\sum_{h} c_{h}\left(1-\rho_{h}\right) Y_{h}}\right]\right\} .
\end{aligned}
$$

But the term in square brackets equals

$$
\begin{aligned}
& \frac{c_{1}\left(1-\rho_{1}\right) X_{1}}{c_{1}\left(1-\rho_{1}\right) Y_{1}\left\{\sum_{h} c_{h}\left(1-\rho_{h}\right) X_{h}+\sum_{h} c_{h}\left(1-\rho_{h}\right) Y_{h}\right\}^{2}} \\
& \times\left[\left(\sum_{h} c_{h}\left(1-\rho_{h}\right) X_{h}+\sum_{h} c_{h}\left(1-\rho_{h}\right) Y_{h}\right)\left(\sum_{h} c_{h}\left(1-\rho_{h}\right) Y_{h}\right)\right. \\
& \left.-\left(\sum_{h} c_{h}\left(1-\rho_{h}\right) X_{h}\right) c_{1}\left(1-\rho_{1}\right) Y_{1}\right]
\end{aligned}
$$

which is positive; so $b_{1}$ is positive.

Now plug (D9) into (D8) to obtain

$$
\sum_{h} b_{h}=\frac{m}{k} c_{1} \bar{\beta}\left\{\rho_{1} \frac{X_{1} Y_{1}}{\left(X_{1}+Y_{1}\right)^{2}}+\left(1-\rho_{1}\right)\left[\frac{K X_{1}}{Y_{1}}-(1-K)^{2}\right]\right\} .
$$

If $Y_{1} /\left(X_{1}+Y_{1}\right)<K$, then $K X_{1} / Y_{1}>(1-K)^{2}$ and $\sum_{h} b_{h}$ is positive. Then, by Theorem D.1,

$$
\frac{\partial X_{1}}{\partial U_{1}}>0
$$

In addition, if $c_{1} \bar{\beta}_{\frac{m}{k}}^{m}<1$, then $\sum_{h} b_{h}>0$ and $\partial X_{1} / \partial U_{1}>0$. 
This work was supported in part by grant RR 02176-01A1 from NIH-DRR, $D H E W$ and in part by a Presidential Initiatives Grant from the University of Michigan. We thank $K$. Dietz, H. Hethcote, $R$. May, and A. Stanley for their critical comments and reviews of this manuscript.

\section{REFERENCES}

1 R. E. F. Matthews, Classification and nomenclature of viruses, Intervirology 12:131-281 (1979).

2 L. S. Kucera, and Q. N. Myrvik, Fundamentals of Medical Virology, 2nd ed., Lea and Febiger, Philadelphia, 1985, Chapter 19, pp. 141-153.

3 F. Barre-Sinoussi, J. C. Chermann, F. Rey, M. T. Nugeyre, S. Chamaret, J. Gruest, C. Dauguet, C. Axler-Blin, F. Vexinet-Brun, C. Rouzioux, W. Rozenbaum, and L. Montagnier, Isolation of a $T$-lymphotropic retrovirus from a patient at risk for acquired immune deficiency (AIDS), Science 220:868-871 (1983).

4 R. C. Gallo, S. Z. Salahuddin, M. Popovic, G. M. Sheaver, M. Kaplan, B. F. Haynes, T. J. Palker, R. Redfield, J. Oleske, B. Safai, G. White, P. Foster, and P. D. Markham, Frequent detection and isolation of cytopathic retroviruses (HTLV-III) from patients with AIDS and at risk for AIDS, Science 224:500-503 (1983).

5 J. L. Marx, Probing the AIDS virus and its relatives, Science 236:1523-1525 (1987).

6 M. A. Gonda, F. Wong-Staal, R. C. Gallo, J. E. Clements, O. Narayan, and R. V. Gilden, Sequence homology and morphologic similarity of HTLV-III and visna virus, a pathogenic lentivirus, Science, 226:173-177 (1985).

7 D. A. Cooper, P. MacLean, R. Finlayson, H. M. Michelmore, J. Gold, B. Donovan, T. G. Barnes, P. Brooks, and R. Penny, Acute AIDS retrovirus infection, Lancet 1985-1:537-540 (1985).

8 R. W. Price, B. Brew, J. Sidtis, M. Rosenblum, A. C. Scheck, and P. Cleary, The brain in AIDS: central nervous system HIV-1 infection and AIDS dementia complex, Science 239:586-592 (1988).

9 J. W. Curran, W. M. Morgan, A. M. Hardy, H. W. Jaffe, W. W. Darrow, and W. R. Dowdle, The epidemiology of AIDS: current status and future prospects, Science 229:1352 1357 (1985).

10 S. Z. Salahuddin, P. D. Markham, R. R. Redfield, M. Essex, J. E. Groopman, M. G. Sarngadharan, M. F. McLane, A. Sliski, and R. C. Gallo, HTLV-III in symptom-free seronegative persons, Lancet 1984-2:1418-1420 (1984).

11 D. P. Francis, P. M. Feorino, J. R. Broderson, H. M. McClure, J. P. Getchell, C. R. McGrath, B. Swenson, J. S. McDougal, E. L. Palmer, A. K. Harrison, F. Barre-Sinoussi, J. C. Chermann, L. Montagnier, J. W. Curran, C. D. Cabradilla and V. S. Kalyanaraman, Infection of chimpanzees with lymphadenopathy-associated virus, Lancet 1984 2:1276-1277 (1984).

12 J. M. A. Lange, D. A. Paul, H. G. Hinsman, F. deWolf, H. van den Berg, R. A. Coutinho, S. A. Danner, J. van der Noordaa, and H. Goudsmit, Persistent HIV antigenaemia and decline of HIV core antibodies associated with transmission of AIDS, Br. Med. J. 293:1459-1462 (1986).

13 G. M. Shaw, M. E. Harper, B. H. Hahn, L. G. Epstein, D. C. Gajdersek, R. W. Price, B. A. Navia, C. K. Petito, C. J. O'Hara, J. E. Groopman, E. S. Cho, J. M. Oleske, F. Wong-Staal, and R. G. Gallo. HTLV-III infection in brains of children and adults with AIDS encephalopathy, Science 227:177-181 (1985). 
14 L. R. Sharer, L. G. Epstein, E. S. Cho, V. Joshi, M. F. Meyenhofer, F. Markus, L. R. Rankin, and C. K. Petito, Pathologic features of AIDS encephalopathy in children: evidence for LAV/HTLV-III infection of brain, Human Pathol. 17:271-284 (1986).

15 B. Navia, B. D. Jordan, and R. W. Price, The AIDS dementia complex: I. Clinical features, Ann. Neurol. 19:517-524 (1986).

16 M. R. Lee, D. D. Ho, and M. E. Gurney, Functional interaction and partial homology between human immunodeficiency virus and neuroleukin, Science 237:1047-1051 (1987).

17 K. J. Lui, D. N. Lawrence, W. M. Morgan, T. A. Peterman, H. W. Haverkos, and D. J. Bregman, A model-based approach for estimating the mean incubation period of transfusion-associated acquired immunodeficiency syndrome, PNAS 83:3051-3055 (1986).

18 K. J. Lui, W. W. Darrow, and G. N. Rutherford III, A model-based estimate of the mean incubation period for AIDS in homosexual men, Science 240:1333-1335 (1988).

19 M. Rees, The sombre view of AIDS, Nature 326:343-345 (1987).

20 G. F. Medley, R. M. Anderson, D. R. Cox, and L. Billard, Incubation period of AIDS in patients infected via blood transfusion, Nature 328:719-721 (1987).

21 J. W. Curran, H. W. Jaffe, A. M. Hardy, W. M. Moprgan, R. M. Selik, and T. J. Dondero, Epidemiology of HIV infection and AIDS in the United States, Science 239:610-616 (1988).

22 J. J. Gonzalez, and M. G. Koch, On the role of "transients" (biasing transitional effects) for the prognostic analysis of the AIDS epidemic, Am. J. Epidemiol. 126:985-1005 (1987).

23 S. W. Lagakos, L. M. Barraj, and V. DeGruttola, Nonparametric analysis of truncated survival data, with application to AIDS, Tech. Report No. 544Z, Dana-Faber Cancer Inst., Boston, 1987.

24 M. Mellye, R. J. Biggar, P. Ebbesen, C. Neuland, J. J. Goedert, V. Faber, I. Lorenzen, P. Skinhoj, R. C. Gallo, and W. A. Blattner, Long-term seropositivity for human $T$-lymphotropic virus type III in homosexual men without the acquired immunodeficiency syndrome: development of immunologic and clinical abnormalities: a longitudinal study, Ann. Intern. Med. 104:496-500 (1986).

25 H. R. Brodt. E. B. Helm. A. Werner, A. Joetten. L. Bergmann, A. Klover, and W. Stille. Spontanverlauf der LAV-HTLV-III-Infektion, Deutsch. Med. Wochenschr. 111:1175-1180 (1986).

26 M. Siekevitz, S. F. Josephs, M. Dukovich, N. Peffer, N. Wong-Staal, and W. C. Greene, Activation of the HIV-1 LTR by T-cello mitogens and the trans-activator protein of HTLV-I, Science 238:1575-1578 (1987).

27 T. M. Folks, J. Justement, A. Kinter, C. A. Dinarello, and A. S. Fanci, Cytokineinduced expression of IIIV- 1 in a chronically infected promonocyte cell line, Science 238:799-802 (1987).

28 L. J. Eales, J. M. Parkin, S. M. Forster, K. E. Nye, J. N. Weber, J. R. W. Harris, and A. J. Pinching, Association of different allelic forms of group specific component with susceptibility to and clinical manifestations of human immunodeficiency virus infection, Lancet 1987-1:999-1002 (1987).

29 T. A. Peterman, D. P. Drotman, and J. W. Curran, Epidemiology of the acquired immunodeficiency syndrome (AIDS), Epidemiol. Rev. 7:1-21 (1985).

30 J. W. Curran, W. M. Morgan, A. M. Hardy, H. W. Jaffe, W. W. Darrow, and W. R. Dowdle, Science 229:1352-1357 (1985). 
31 S. H. Landesman, H. M. Ginzburg, and S. H. Weiss, The AIDS epidemic, $N$. Engl. $J$. Med. 312:521-525 (1985).

32 H. W. Jaffe, W. W. Darrow, D. F. Echenberg, P. J. O'Malley, J. P. Getchell, V. S. Kalyanaraman, R. H. Byers, D. P. Drennan, E. H. Braff, J. W. Curran, and D. P. Francis, The acquired immunodeficiency syndrome in a cohort of homosexual men, Ann. Intern. Med. 103:210-214 (1985).

33 R. R. Redfield, P. D. Markham, S. Z. Salahuddin, M. G. Sarngadharan, A. J. Brodner, T. M. Folks, W. R. Ballou, D. C. Wright, and R. C. Gallo, Frequent transmission of HTLV-III among spouses of patients with AIDS-related complex and AIDS, JAMA 253:1571-1573 (1985).

34 C. Harris, C. B. Small, R. S. Klein, G. H. Friedland, B. Moll, E. E. Emeson, I. Spigland, and N. H. Steigbigel, Immunodeficiency in female sexual partners of men with acquired immunodeficiency syndrome, $N$. Engl. J. Med. 308:1181-1184 (1983).

35 G. Luzi, B. Ensoli, G. Turbessi, B. Scarpati, and F. Auiti, Transmission of HTLV-III infection by heterosexual contact, Lancet 1985-2:1018 (1985).

36 G. A. Papaevangelou, A. Roumeliotu-Karayannis, G. Kallinikos, and G. Papoutsakis, LAV/HTLV-III infection in female prostitutes, Lancet 1985-2:1018 (1985).

37 D. C. DesJarlais, S. R. Friedman, and R. L. Stoneburner, HIV infection and intravenous drug use: critical issues in transmission dynamics, infection outcomes, and prevention, Rev. Infect. Dis. 10:151-158 (1988).

38 W. Winkelstein, Jr., D. M. Lyman, N. Padian, R. Grant, M. Samuel, J. A. Wiley, R. E. Anderson, W. Lang, J. Riggs, and J. A. Levy, Sexual practices and the risk of infection by the human immunodeficiency virus, JAMA 257:321-325 (1987).

39 N. Padian, J. Wiley, and W. Winkelstein, Male to female transmission of human immunodeficiency virus: current results, infectivity rates, and San Francisco population seroprevalence estimates, presented at the III Int. Conf. on AIDS, Washington, D.C., 1987.

40 R. M. Grant, J. A. Wiley, and W. Winkelstein, Infectivity of the human immunodeficiency virus: estimates from a prospective study of homosexual men, J. Infect. Dis. 156:189-193 (1987).

41 W. Winkelstein, D. M. Lyman, N. Padian, R. Grant, M. Samuel, J. A. Wiley, R. E. Anderson, W. Lang, J. Riggs, and J. A. Levy, Sexual practices and risk of infection by the human immunodeficiency virus, JAMA 257:321-325 (1987).

42 T. A. Peterman, R. L. Stoneburner, J. R. Allen, H. W. Jaffe, and J. W. Curran, Risk of human immunodeficiency virus transmission from heterosexual adults with transfusion associated infections, JAMA 259:55-58 (1988).

43 J. Pickering, J. A. Wiley, N. S. Padian, L. E. Lieb, D. G. Echenberg, and J. Walker, Modeling the incidence of acquired immunodeficiency syndrome (AIDS) in San Francisco, Los Angeles and New York, Math. Modeling 7:661-688 (1986).

44 N. T. J. Bailey, The Mathematical Theory of Infectious Diseases, 2nd ed., Hafner, New York, 1975.

45 K. Dietz, Epidemics and rumours: A survey, J. Roy. Stat. Soc. Ser. A 130:505-528 (1967).

46 H. Hethcote, Qualitative analysis of communicable disease models, Math. Biosci. 28:335-356 (1976).

47 K. Dietz and D. Schenzle, Mathematical models for infectious disease statistics, in $A$ Celebration of Statistics. The ISI Centenary Volume, A. C. Atkinson and S. E. Feinberg, eds., Springer-Verlag, New York, 1985, pp. 167-204. 
48 R. M. Anderson and R. M. May, Population biology of infectious diseases. Part I, Nature 280:361-367 (1979).

49 R. M. May, and R. M. Anderson, Population biology of infectious diseases. Part II, Nature 280:455-461 (1979).

50 R. M. Anderson and R. M. May, Vaccination against rubella and measles: quantitative investigations of different policies, J. Hyg. 90:259-325 (1983).

51 A. Nold, Heterogeneity in disease-transmission modeling, Math. Biosci. 52:227-240 (1980).

52 D. W. Tudor, An age-dependent epidemic model with application to measles, Math. Biosci. 73:131-147 (1985).

53 H. W. Hethcote, and J. W. Van Ark, Epidemiological models for heterogeneous populations: proportionate mixing, parameter estimation, and immunization programs, Math. Biosci. 84:85-118 (1987).

54 R. M. Anderson, and R. M. May, Age-related changes in the rate of disease transmission: implications for the design of vaccination programmes, J. Hyg. 94:365-436 (1985).

55 R. M. May and R. M. Anderson, Spatial heterogeneity and the design of immunization programs, Math. Biosci. 72:83-111 (1984).

56 C. C. Travis and S. M. Lenhart, Eradication of infectious diseases in heterogeneous populations, Math. Biosci. 83:191-198 (1987).

57 L. Sattenspiel, Population structure and the spread of disease, Human Biol. 59:411-438 (1987).

58 L. Sattenspiel and C. P. Simon, The spread and persistence of infectious diseases in structured populations, Math. Biosci. 90:341-366 (1988).

59 I. M. Longini, Jr., E. Ackerman, and L. R. Elveback, An optimization model for influenza A epidemics, Math. Biosci. 38:141-157 (1978).

60 I. M. Longini, Jr. and J. S. Koopman, Houschold and community transmission parameters from final distributions of infections in households, Biometrics 38:114-126 (1982).

61 I. M. Longini, Jr., J. S. Koopman, A. S. Monto, and J. P. Fox, Estimating household and community transmission parameters for influenza, Am. J. Epidemiol. 115:736-751 (1982).

62 I. M. Longini, Jr., J. S. Koopman, M. Haber, and G. A. Cotsonis, Statistical inference for infectious diseases: risk-specific household and community transmission parameters, Am. J. Epidemiol. (in press).

63 A. Lajmanovich, and J. A. Yorke, A deterministic model for gonorrhea in a nonhomogeneous population, Math. Biosci. 28:221-236 (1976).

64 H. W. Hethcote, and J. A. Yorke, Gonorrhea Transmission Dynamics and Control, Lecture Notes in Biomathematics, No. 56, Springer-Verlag, Heidelberg, 1984.

65 E. G. Knox, A transmission model for AIDS, Eur. J. Epidemiol. 2:165-177 (1986).

66 D. S. Kiessling, S. Stannat, I. Schedel, and H. Deicker, Uberlegungen und hochrechnungen zur epidiologie des "acquired immunodeficiency syndrome" in der Bundesrepublik Deutschland, Infection 14:217-221 (1986).

67 R. M. May and R. M. Anderson, Transmission dynamics of HIV infection, Nature 326:137-142 (1987).

68 R. M. Anderson, G. F. Medley, R. M. May, and A. M. Johnson, A preliminary study of the transmission dynamics of the human immunodeficiency virus (HIV), the causative agent of AIDS, IMA J. Math. Med. Biol. 3:229-263 (1986). 
69 R. M. Anderson, S. P. Blythe, G. F. Medley, and A. M. Johnson, Is it possible to predict the minimum size of the acquired immunodeficiency syndrome (AIDS) epidemic in the United Kingdom? Lancet 1987-1:1073-1075 (1987).

70 J. M. Hyman and E. A. Stanley, Using mathematical models to understand the AIDS epidemic, Math. Biosci. 90:415-473 (1988).

71 K. Deitz, On the transmission dynamics of HIV, Math. Biosci. 90:397-414 (1988).

72 H. W. Hethcote, A model for HIV transmission and AIDS, to appear.

73 C. Castillo-Chavez, K. Cooke, W. Huang, and S. A. Levin, The role of long periods of infectiousness in the dynamics of acquired immunodeficiency syndrome (AIDS). in: Mathematical Approaches to Environmental and Ecological Problems, Proceedings of a Cornell University Conference, C. Castillo-Chavez, S. A. Levin, and C. Shoemaker, eds., Lecture Notes in Biomathematics, Springer-Verlag. New York, 1988.

74 R. M. Anderson, R. M. May, and A. R. McLean, Possible demographic consequences of AIDS in developing countries, Nature 332:228-234 (1988).

75 R. M. May, personal communication (1988).

76 J. A. Jacquez, Compartmental Analysis in Biology and Medicine, 2nd ed., Univ. of Michigan Press, Ann Arbor, 1985.

77 H. W. Hethcote, J. A. Yorke, and A. Nold, Gonorrhea modeling: a comparison of control methods, Math. Binsci. 58:93-109 (1982).

78 R. M. May, R. M. Anderson, and A. R. McLean, Possible demographic consequences of HIV/AIDS epidemics: I, assuming HIV infection always leads to AIDS, Math. Biosci. (in press) 1988.

79 R. M. May, R. M. Anderson, and A. R. McLean, Possible demographic consequences of HIV/AIDS epidemics: II, assuming HIV infection does not necessarily lead to AIDS, in Mathematical Approaches to Ecological and Environmental Problems, C. Castillo-Chavez, S. A. Levin, and C. Shoemaker, eds., Lecture Notes in Biomathematics, Springer-Verlag, New York, 1988.

$80 \mathrm{~K}$. Dietz, Transmission and control of arbovirus diseases, in Epidemiology, D. Ludwig and K. L. Clarke, eds., SIAM, Philadelphia, 1976.

81 G. MacDonald, The analysis of equilibrium in malaria, Tropical Dis. Bull. 49:813-829 (1952).

82 R. M. Anderson and R. M. May, Population Biology of Infectious Diseases, Dahlem Workshop Report, Springer-Verlag, New York, 1982.

83 K. Dietz, Mathematical models for infectious diseases; evaluation of measures for prevention and control, in Perspectives in Medical Statistics, J. F. Bithell and R. Coppi, eds., Academic, New York, 1981.

84 R. M. May and R. M. Anderson, The transmission dynamics of human immunodeficiency virus (HIV), Proc. R. Soc. (in press).

85 M. Berman and M. F. Weiss, SAAM Manual, U.S. DHEW Publication No. 78-180 (NIH), Washington, D.C., 1978.

86 M. Berman, W. F. Beltz, P. C. Greif, R. Chabay, and R. C. Boston, CONSAM User's Guide, USDHEW, NIH Lab. for Math. Biology, 1983.

87 B. Richmond, S. Peterson, and P. Vescuso, An Academic User's Guide to STELLA, High Performance Systems, Lyme, N.H., 1987.

88 E. Ackerman, L. R. Elveback, and J. P. Fox, Simulation of Infectious Disease Epidemics, C. C. Thomas, Springfield, Ill., 1984.

89 H. Hethcote, An immunization model for a heterogeneous population, Theor. Pop. Biol. 14:338-349 (1978). 
90 W. M. Post, D. L. DeAngelis, and C. C. Travis, Endemic disease in environments with spatially heterogeneous host populations, Math. Biosci. 63:289-302 (1983).

91 H. Hethcote, and H. R. Thieme, Stability of the endemic equilibrium in epidemic models with subpopulations, Math. Biosci. 75:205-277 (1985).

92 C. Clark, Mathematical Biosciences, Wiley, New York, 1976.

93 C. Coleman, Quadratic population models: almost never any cycles, in Differential Equation Models, M. Braun, C. Coleman, and D. Drew, eds., Springer-Verlag, New York, 1983.

94 C. Olech, On the global stability of autonomous systems in the plane, Contrib. Diff. Equations I:389-400 (1963).

95 W. Feller, Introduction to Probability Theory and Its Applications, Wiley, New York, 1968, p. 242.

96 A. Takayama, Mathematical Economics, Dryden Press, Hinsdale, Ill., 1974.

97 C. P. Simon, Some fine-tuning for dominant diagonal matrices, University of Michigan Preprint, 1988 